2014

\title{
MULTI-WAVELENGTH SPECKLE REDUCTION FOR LASER PICO- PROJECTORS USING DIFFRACTIVE OPTICS
}

Weston $\mathrm{H}$. Thomas

Michigan Technological University

Follow this and additional works at: https://digitalcommons.mtu.edu/etds

Part of the Electrical and Computer Engineering Commons, and the Optics Commons Copyright 2014 Weston $\mathrm{H}$. Thomas

\section{Recommended Citation}

Thomas, Weston H., "MULTI-WAVELENGTH SPECKLE REDUCTION FOR LASER PICO-PROJECTORS USING DIFFRACTIVE OPTICS", Dissertation, Michigan Technological University, 2014.

https://doi.org/10.37099/mtu.dc.etds/860

Follow this and additional works at: https://digitalcommons.mtu.edu/etds

Part of the Electrical and Computer Engineering Commons, and the Optics Commons 


\title{
MULTI-WAVELENGTH SPECKLE REDUCTION FOR LASER PICO-PROJECTORS USING DIFFRACTIVE OPTICS
}

By

Weston H. Thomas

\begin{abstract}
A DISSERTATION
Submitted in partial fulfillment of the requirements for the degree of

DOCTOR OF PHILOSOPHY

In Electrical Engineering
\end{abstract}

MICHIGAN TECHNOLOGICAL UNIVERSITY

2014

(C) 2014 Weston H. Thomas 
This dissertation has been approved in partial fulfillment of the requirements for the Degree of DOCTOR OF PHILOSOPHY in Electrical Engineering.

Department of Electrical and Computer Engineering

Dissertation Advisor: Dr. Christopher Middlebrook

Committee Member: Dr. Michael Roggemann

Committee Member: $\quad$ Dr. Warren Perger

Committee Member: Dr. Ramy El-Ganainy

Department Chair/School Dean: Dr. Daniel R. Fuhrmann 
To my mom and dad who never doubted my ability to succeed and who went above and beyond to support my dreams.

Special thanks to my advisor, Dr. Middlebrook, for seeing my potential and pushing me to achieve it. 


\section{Table of Contents}

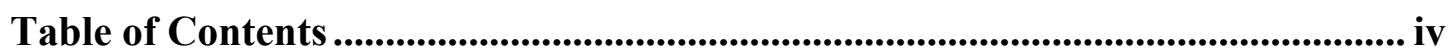

List of Figures......................................................................................................................... viii

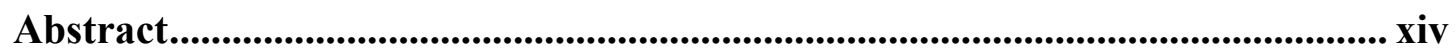

Chapter 1: Introduction ............................................................................................. 1

1.1 Speckle Contrast Measurement.................................................................................. 3

1.2 Speckle Reduction Techniques .................................................................................... 4

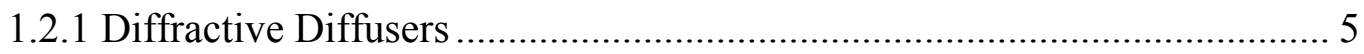

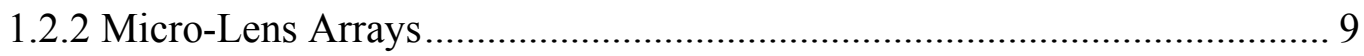

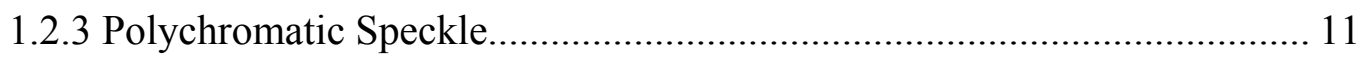

1.4 Research Outline ........................................................................................................... 11

Chapter 2: Speckle Theory ………………........................................................................ 14

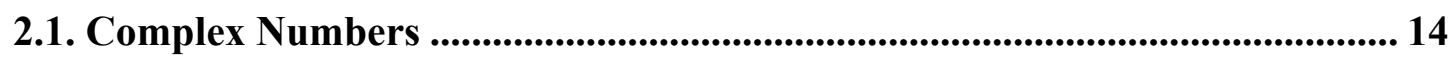

2.2. Random Phasors ................................................................................................................ 18

Chapter 3: Diffractive Diffusers............................................................................ 24 
3.2. Measurement Setup 26

3.3. Speckle Contrast Results 30

3.4. Rotating Diffuser Conclusion. 36

Chapter 4: Secondary Speckle Reduction Methods 38

4.1. Introduction to Laser Diodes and Fiber Optics 38

4.2. Speckle Contrast Results 41

4.3. Polarization Based Speckle Effects. 47

4.4. Secondary Speckle Reduction Methods' Conclusion 50

Chapter 5: Simulating Diffusers using Fourier Optics 51

5.1. Simulation Requirements 51

5.2. Fourier Optical Model for Diffusers 55

5.3. Modeling the Diffusers 60

5.4. Speckle Contrast Results 69

5.5. Diffuser Simulation Conclusion.

Chapter 6: Simulation and Creation of New Diffuser Design 75 
6.2. Theoretical Analysis................................................................................. 78

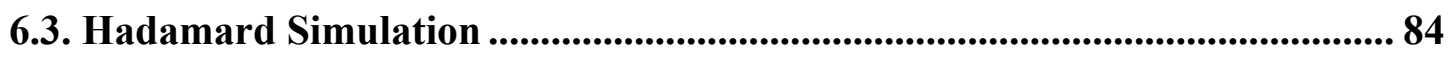

6.4. Experimental Results and Comparison ................................................................. 87

6.5. Dual Hadamard Diffuser Conclusion............................................................90

Chapter 7: RGB Application of Hadamard Diffusers...................................... 91

7.1. Polychromatic Speckle Introduction.................................................................... 91

7.2. Wavelength in Diffractive Optics ........................................................................ 92

7.3. Measuring RGB Speckle Contrast Images...................................................... 95

7.4. Diffusers Separation Distance.................................................................. 101

7.5. Polychromatic Speckle Reduction Conclusion ........................................... 104

Chapter 8: Conclusion ........................................................................................................ 105

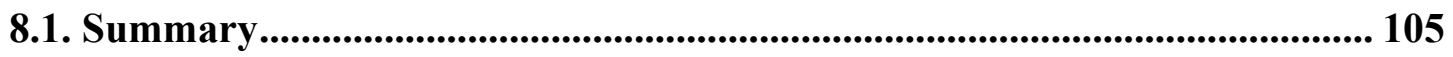

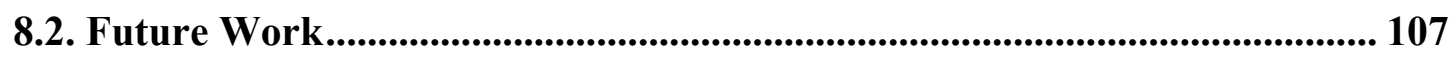

References................................................................................................................................. 108

Appendix A: Hadamard Matrices ................................................................ A-1 
Appendix B: MATLAB Code for Monochromatic Dual Hadamard Diffuser

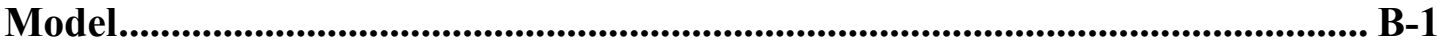

Appendix C: MATLAB Code for Polychromatic Dual Hadamard Diffuser

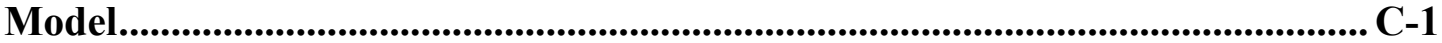




\section{List of Figures}

Figure 1.1: Example of imaged speckle from monochromatic laser source............. 2

Figure 1.2: Generic a) amplitude rectangular grating profile b) phase grating

profile (phase front) ......................................................................................................... 7

Figure 1.3: MLA illuminated with $535 \mathrm{~nm}$ laser light a) full image and b) zoomed

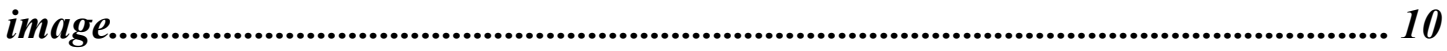

Figure 2.1: Young's double slit experiment diagram............................................... 17

Figure 2.2: Interference fringes in circular laser beam structure............................ 18

Figure 2.3: Demonstration of speckle by photon combination ............................... 18

Figure 2.4: Random phasors contribute to single phasor total................................ 19

Figure 3.1: Speckle Image caused by laser incident on a) grayscale diffuser b)

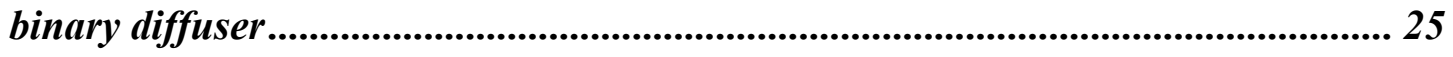

Figure 3.2: Diffuser image with metric dimensions.................................................. 26

Figure 3.3: a) Optical bench top layout and b) diffuser rotational mount............. 28

Figure 3.4: Rotation diffuser experimental setup layout ........................................ 29 
Figure 3.5: Comparison of Beam Sizes using 07-133-02 Grayscale Diffuser (at 532nm) 30

Figure 3.6: Comparison of Beam Sizes using 07-096-01 Binary Diffuser (at $632 \mathrm{~nm})$. 31

Figure 3.7: Comparison of Beam Sizes using 07-133-02 Grayscale Diffuser $(532 \mathrm{~nm})$ 31

Figure 3.8: Comparison of Beam Sizes using 07-096-01 Binary Diffuser (532nm)32

Figure 3.9: Comparison of Polarization States for Varying Beam Sizes and Speeds using 07-096-01 Binary Diffuser (532nm). 33

Figure 3.10: Comparison of Polarization states for varying beam size and speed using 07-133-02 Grayscale Diffuser (532nm).

Figure 3.11: a) Stationary speckle (grayscale) image and b) Rotational diffuser speckle image (at $90 \mathrm{~Hz}$ ) 35

Figure 3.12: Diagonal cross section of speckled images (Beam $2.99 \mathrm{~mm}$ ) with and without a) binary diffuser (07-096-01); b) grayscale diffuser (07-133-02) 36

Figure 4.1: Fiber optic speckle with a) Stationary binary diffuser b) Rotating binary diffuser at $60 \mathrm{~Hz}$ 44

Figure 4.2: Fiber optic speckle with a) Stationary grayscale diffuser and b) Rotating grayscale diffuser at $60 \mathrm{~Hz}$ 45 
Figure 4.3: Fiber mode image without diffuser..

Figure 4.4: Graph of Comparison between the three laser wavelengths/sources with red and green laser values taken from Chapter 2..................................................... 47

Figure 4.5: Split polarization optical setup........................................................ 49

Figure 4.6: Split polarization speckle image with a) a grayscale diffuser and b) a

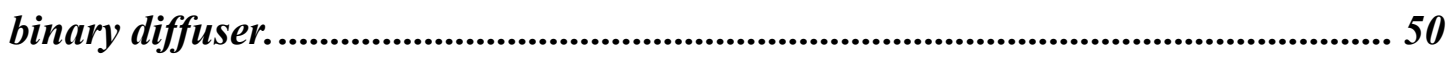

Figure 5.1:(a) Diffuser model layout; (b) Diffuser unit cell layout.......................... 56

Figure 5.2: Gaussian Beam with linear width of $1.5 \mathrm{~mm}$...................................... 57

Figure 5.3: (a) Grayscale diffuser array; (b) Binary diffuser array...................... 61

Figure 5.4: Simulated output of binary diffuser with phase levels of a) $\pi / 2 \&-\pi / 2$

b) $\pi / 4 \&-\pi / 4$ c) $\pi / 8 \&-\pi / 8$

Figure 5.5: Generic Grayscale diffuser model at $10 \mathrm{~cm}$ 65

Figure 5.6: a) Under-sampling caused by element size; b) Incorrect scaling of

Fresnel propagator..................................................................................................... 67

Figure 5.7: In-proper scaling/sampling of Grayscale Diffuser ............................ 68

Figure 5.8: Rotating speckle image with a) $\Delta \theta=50$ b) $\Delta \theta=0.75 \ldots \ldots . . . \ldots . . . . . . . . . . .69$

Figure 5.9: (a) Stationary binary diffuser; (b) Stationary grayscale diffuser......... 70 
Figure 5.10: (a) Focused, stationary binary diffuser; (b) Focused, stationary grayscale diffuser; (c) Physical binary diffuser output intensity image; (d) Physical grayscale diffuser output intensity image ................................................................... 71

Figure 5.11: (a) Rotating binary diffuser; (b) Rotating grayscale diffuser............. 72

Figure 5.12: (a) Diffuser model with uniform illumination; (b) Diffuser model with Gaussian illumination 73

Figure 6.1: Order 16 Sylvester Matrix 77

Figure 6.2: Current diffuser layout using projection lens

Figure 6.3: Single diffuser simulation results, a) Intermediate Image Screen, b)

Final Image Screen, c) Final Image normalized... 85

Figure 6.4: Dual diffuser simulation results, a) Image directly after second diffuser, b) Final image output from dual diffuser system, c) Close up image output from dual diffuser system ................................................................................ 87

Figure 6.5: Physical Hadamard diffuser - image capture ..................................... 88

Figure 6.6: Dual Hadamard diffusers - image capture 89

Figure 7.1: Simulated (left) and experimental (right) speckle images from DHDs with 535 nm laser light 92 
Figure 7.2: Simulated dual Hadamard diffusers at a) $635 \mathrm{~nm}$, b) $535 \mathrm{~nm}$, c) 450 $n m$, d) 635, 535, and $450 \mathrm{~nm}$ overlaid together

Figure 7.3: Multi-wavelength speckle setup using a) a single DHD b) multiple DHD. 96

Figure 7.4: Simulated RGB DHD in a) grayscale, b) averaged RGB image, c) full color RGB image. 98

Figure 7.5: Optical bench top setup images, a) \& b). 100

Figure 7.6: Spectral response curve for DC210 (Thorlabs)

Figure 7.7: Bench top speckle image using a) grayscale detection and b) RGB detection. 101

Figure 7.8: Separation distance between the DHD for all three wavelengths: a) at $635 \mathrm{~nm}, 535 \mathrm{~nm}$ and 450; b) Full color using grayscale and RGB detection 103

Figure A.1: Order 16 a) Sylvester matrix, b) Walsh-Paley matrix, c) Walsh matrix

Figure A.2: Sylvester matrix circular diffuser.

Figure A.3: a) Sylvester matrix diffuser image; b) Walsh-Paley matrix diffuser image; c) Walsh matrix diffuser image. 
Figure A.4: Rotating (60 cycles/s) a) Sylvester diffuser, b) Walsh-Paley diffuser, c)

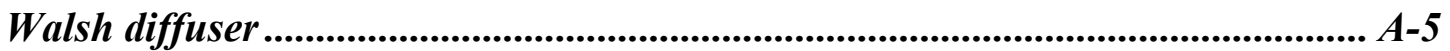




\section{Abstract}

Personal electronic devices, such as cell phones and tablets, continue to decrease in size while the number of features and add-ons keep increasing. One particular feature of great interest is an integrated projector system. Laser pico-projectors have been considered, but the technology has not been developed enough to warrant integration. With new advancements in diode technology and MEMS devices, laser-based projection is currently being advanced for pico-projectors. A primary problem encountered when using a pico-projector is coherent interference known as speckle. Laser speckle can lead to eye irritation and headaches after prolonged viewing. Diffractive optical elements known as diffusers have been examined as a means to lower speckle contrast. Diffusers are often rotated to achieve temporal averaging of the spatial phase pattern provided by diffuser surface. While diffusers are unable to completely eliminate speckle, they can be utilized to decrease the resultant contrast to provide a more visually acceptable image.

This dissertation measures the reduction in speckle contrast achievable through the use of diffractive diffusers. A theoretical Fourier optics model is used to provide the diffuser's stationary and in-motion performance in terms of the resultant contrast level. Contrast measurements of two diffractive diffusers are calculated theoretically and compared with experimental results. In addition, a novel binary diffuser design 
based on Hadamard matrices will be presented. Using two static in-line Hadamard diffusers eliminates the need for rotation or vibration of the diffuser for temporal averaging. Two Hadamard diffusers were fabricated and contrast values were subsequently measured, showing good agreement with theory and simulated values. Monochromatic speckle contrast values of 0.40 were achieved using the Hadamard diffusers.

Finally, color laser projection devices require the use of red, green, and blue laser sources; therefore, using a monochromatic diffractive diffuser may not optimal for color speckle contrast reduction. A simulation of the Hadamard diffusers is conducted to determine the optimum spacing between the two diffusers for polychromatic speckle reduction. Experimental measured results are presented using the optimal spacing of Hadamard diffusers for RGB color speckle reduction, showing $60 \%$ reduction in contrast. 


\section{Chapter 1: Introduction}

As technology continues to increase in complexity, the requirements for electronics to decrease in size is intensified. New designs for pico-projectors have been a common theme for the last decade and laser diodes are an ideal light source for this application. Laser sources provide a larger color gamut and brighter light than LEDs, while still requiring less power to operate. The second motivation is the size of the laser diode and its ability to have infinite focus compared to standard projection lamps, which would allow for much smaller, mobile projector systems. While lasers are ideal for integration into hand held devices such as pico-projectors, they are also the preferred light sources for large scale projectors. The large down side associated with lasers is an effect known as speckle. Speckle is a grainy spot pattern that appears in images created by coherent light sources due to self-interference. Interference is an effect resulting from multiple light beams overlapping and can be positive (constructive interference) or negative (destructive interference) [1]. In order for interference to occur the light beams must be coherent and have the same frequency. The pattern that is created from the interference of two beams of light is often referred to as a fringe [1]. Interference fringes create an irradiance pattern of constant bright and dark regions [1]. Speckle is a specific form of interference resulting from multiple instances of fringes occurring at the same point in time and 2-D space, resulting in a spotted pattern. An example of a speckle pattern is given in Figure 1.1. 
While laser diodes have benefits, their use in projectors is limited due to speckle which hampers the formation of projected images.

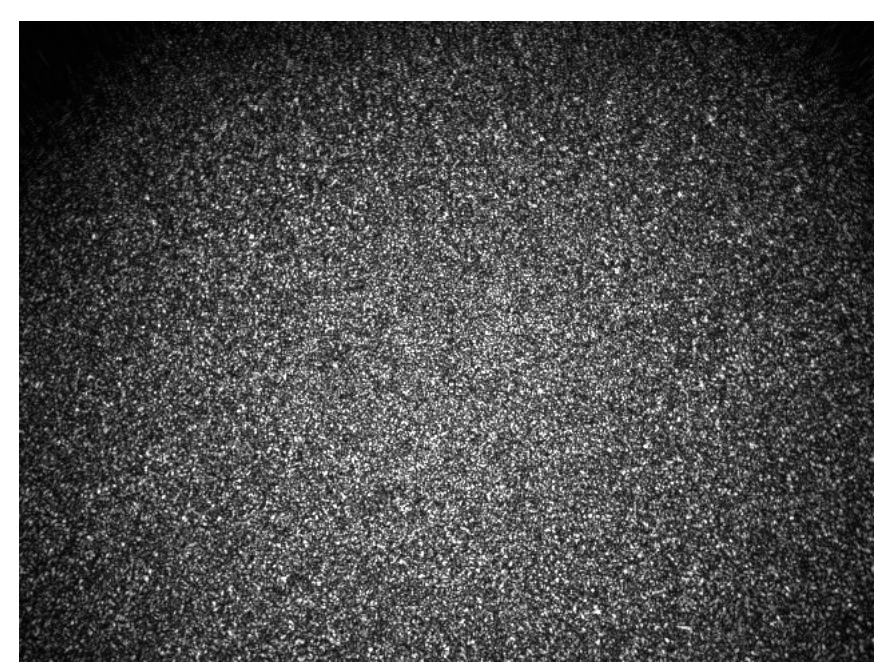

Figure 1.1: Example of imaged speckle from monochromatic laser source

Laser coherence refers to each generated wave of light exiting from a source as being in phase with one another. Laser coherence can be separated into two classifications: temporal coherence and spatial coherence. Temporal coherence describes the wave of light having the same phase over a period of time (coherence time), while spatial coherence is defined as each photon having the same phase over a specific spatial region (coherence area). If the coherence time is large, the wave of light will have a correspondingly high temporal coherence. The linewidth of the laser source is inversely related to the temporal coherence of the source. As an example, a perfectly monochromatic source would have an "infinite" coherence length and would never lose temporal coherence. Laser diodes tend to have longer coherence lengths (high temporal coherence) since they have narrower linewidths [2]. Both the temporal and spatial coherence of a laser will decrease as the laser continues to propagate from the source. Temporal coherence decreases the farther from the source along the optical 
axis the light travels, while spatial coherence is lessened as the laser expands outwards in any direction perpendicular to the direction of propagation. Spatial coherence is often overlooked because of the relationship between the temporal coherence and the linewidth of the laser. However, speckle will be reduced when either or both components (temporal or spatial) are diminished. Completely destroying or removing the coherence of a laser source would solve the speckle "problem" since self-interference would no longer be possible, but a laser without coherence is simply a LED. A LED, while small enough to fit in handheld devices, has finite focus requiring a more complex optical system for projector design. In order to realize a pico-projector utilizing laser sources, it is necessary to partially reduce the coherence of the light wave to reduce the subsequent speckle. Achieving this goal will allow for a compact, low power, and bright light source for picoprojectors. Utilizing non-moving Hadamard diffractive optical elements will allow for polychromatic speckle reduction in pico-projector applications.

\subsection{Speckle Contrast Measurement}

In order to evaluate the reduction of speckle a standard by which various speckled images can be compared is needed. Speckle reduction is measured by the speckle contrast between the dark and bright spots associated with the pattern as shown in Figure 1.1. The speckle specific contrast metric is defined by Equation 1.1 [3].

$$
C=\frac{\sigma_{I}}{I_{\text {Mean }}}=\frac{1}{S N R}
$$


The standard deviation of the irradiance, $\sigma_{I}$, is found over the spatial area of the speckle pattern, as is the average irradiance, $I_{\text {Mean }}$. The contrast of a speckle pattern is also inversely related to the signal-to-noise ratio, $S N R$, given by the same speckle image. The standard deviation and average irradiance of the speckle pattern must be calculated before completing the speckle contrast measurement.

\subsection{Speckle Reduction Techniques}

Speckle reduction research has been commonplace in journals and proceedings since the first paper detailing speckle in 1962 [4]. Speckle contrast is decreased by the number of degrees of freedom associated with the speckle reduction technique that is being applied. This is calculated by Equation 1.2, where $M$ is the number of degrees of freedom [5]. Multiple speckle reduction methods can be used in combination and the overall contrast is found by adding up the total degrees of freedom, $M$ of all the setups.

$$
C=\frac{1}{\sqrt{M}}
$$

The degrees of freedom of a speckle pattern can include the number of unique speckle patterns overlaid on top of one another. Speckle illuminated onto a depolarized surface results in two degrees of freedom associated with the speckle and likewise a contrast reduction of $\frac{1}{\sqrt{2}}$ [5]. No individual speckle reduction technique has yet been effective at totally eliminating speckle. It is generally thought that multiple techniques working in conjunction are necessary for full contrast reduction. Such common techniques involve temporal averaging using moving/changing diffuser, further 
temporal coherence reduction using fiber optic bundle with disassociated fiber lengths, and illumination using multiple non-coherent sources/wavelength diversity. Manipulating the polarization in various ways also provides a benefit as $\mathrm{S} \& \mathrm{P}$ polarizations can create multiple non-interfering images [5].

\subsubsection{Diffractive Diffusers}

Speckle can also be reduced by transmitting coherent illumination through a phase screen. Phase screens, known as diffusers, can be used to offset the original coherence in the laser light prior to reflection from a surface. The degraded coherent optical field results in a change of the image irradiance, thus resulting in a change of the speckle contrast. A diffractive diffuser is merely a specific type of diffraction grating and can provide the speckle contrast reduction necessary for a laser projection system. Diffraction gratings come in various shapes and sizes and are used for a multitude of purposes, including beam shaping [6], focusing [7] and, in the case previously specified, adjusting the coherence structure of the laser [5].

A diffraction grating is a periodic structure that causes alterations in the amplitude or phase (or both) of the incident light. Diffraction gratings can either be reflective, light reflects from the grating surface, or transmissive, light passes through the grating surface. The reflected or transmitted light from the grating will form spatial orders of light based on the period structure of the grating and the wavelength of the incident light. The angular output of the orders from a 1-dimensional grating with incoming light at normal incidence to the surface of the grating will be governed by the following: [8] 


$$
\sin \theta=\frac{m \lambda}{d}
$$

where $\lambda$ is wavelength, $\theta$ is the angle of the order, $m$ identifies the order value (integer), and $d$ is periodicity of the grating. Incident light not perpendicular to the normal will have an angular dependence based on the incoming light. Knowing information concerning the diffractive orders will allow calculations of the distributed intensity across each of the orders. Each order that appears will have a percentage of the total power incident on the grating. The irradiance of each order can be calculated and is a direct result of the periodic structure associated with the diffraction gratings. The diffraction efficiency can then be calculated by the ratio of incident power to total output power of the grating. A more advanced grating has structure elements in 2 linear directions, also known as a plane or surface grating.

The diffractive effect of the grating can be defined in two ways: by phase or by amplitude. An amplitude grating will either pass a fractional value of the incident field amplitude or stop it. Meanwhile, a phase grating will adjust the phase component of the incident light field in such a way as to create an interference pattern, causing a distinct intensity pattern. A linear amplitude pattern is shown in Figure 1.2a and a linear phase grating can be seen in Figure 1.2b.

In the case of an amplitude grating, the transmittance function is defined by the actual pattern. However, in a phase grating the transmittance function is caused by the exponential value of the described pattern, Equation 1.4 [9]. 


$$
t_{A}=e^{\varphi(x)}
$$

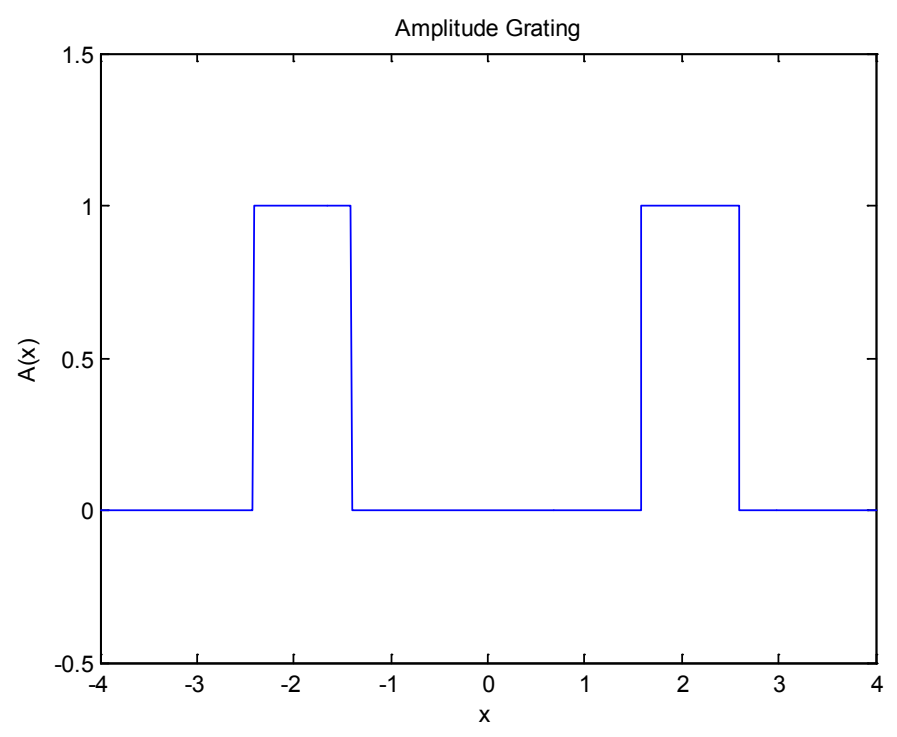

a)

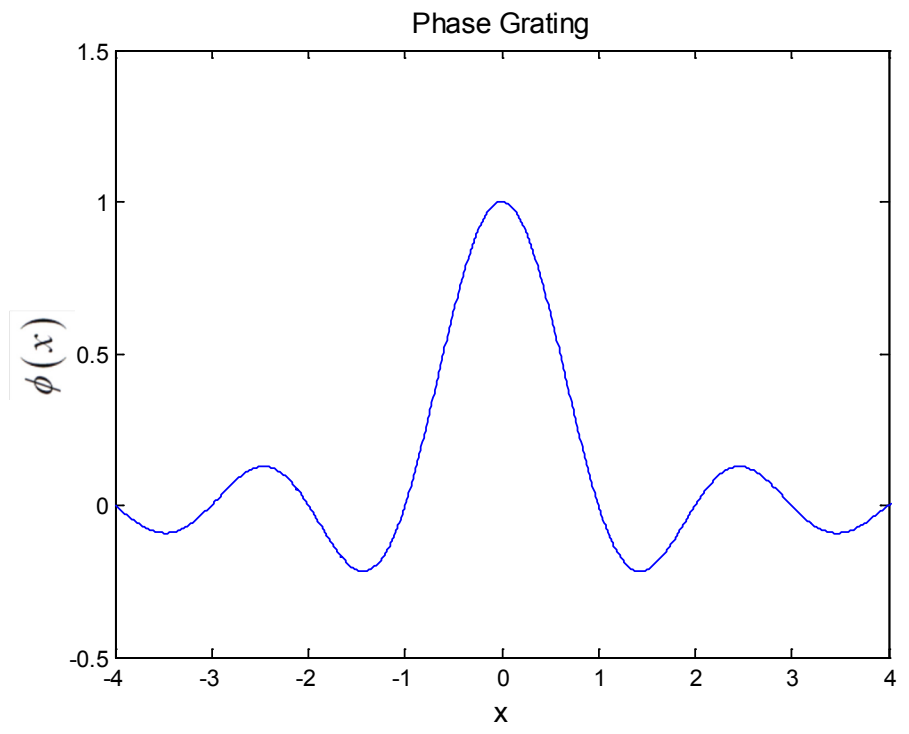

b)

Figure 1.2: Generic a) amplitude rectangular grating profile b) phase grating profile (phase front)

Phase gratings have the property of increasing points of interference in the image plane, rather than just allowing light to pass. The interference (destructive or constructive) for small phase disruptions from the phase grating will appear as 
speckle. Certain phase gratings are designed to create specific speckle patterns in an attempt to reduce naturally occurring speckle from an incident surface. Phase grating based diffusers are the most common diffractive grating used in speckle reduction. Two main types of diffusers will be discussed: binary and grayscale. These two diffuser types will be investigated further in Chapter 2. Both diffractive gratings will have separate orders that occur with distributed intensity as discussed previously. Diffusers used for speckle reduction are often designed so that $90 \%$ or more of the power is passed into the first diffractive order. Having the majority of power in the first order allows the designer to focus their efforts into optimizing the speckle reduction that occurs only in the first order.

Diffractive diffusers have been a popular option for speckle reduction for much of the last decade. A well-known speckle reduction technique is rotation or vibration of the diffuser, which allows temporal averaging to occur. Stationary diffusers provide a known structured phase pattern which creates a predictable irradiance pattern. Moving the diffuser introduces multiple instances of the phase pattern at the same point in space. If the multiple phase patterns overlap faster than the eye integrates at $23 \mathrm{~Hz}$ [10], the final irradiance image is an averaged version of combined phase patterns. The speckle reduction that occurs from temporal averaging is based on the number of instantaneous phase patterns created by the diffuser. Investigations have been made into optimizing a rotating diffuser and speckle contrast values of 0.09 have occurred previously [11-14] . 
As previously mentioned, reduction in the temporal coherence of a laser diode also reduces the speckle. This can be accomplished by introducing laser light to a bundle fiber with each individual fiber having a differing length. The irradiance from the recombined fiber bundle results in a number of "out of phase" light patterns which results in an lower speckle contrast [5]. Optical fibers have also been used to break the spatial coherence of the light through high modal separation within the fiber itself [15]. Using highly multi-mode fibers, mode mixing can be created by vibration or bending which breaks up the wave-front of the incident beam causing coherence reduction [16]. Another method is using multiple light sources with orthogonal polarization to eliminate interference in the irradiance pattern and reduces the perceived speckle [17]. Amongst the many speckle reduction approaches the use of diffractive diffusers remains one of the most prevalent methods for speckle reduction.

\subsubsection{Micro-Lens Arrays}

A benefit of diffractive diffusers is that they work well in conjunction with other linear optical elements that could be needed for pico-projectors. A micro-lens array (MLA) is used to shape laser beams into a uniform pattern that conforms to the size and shape of the spatial light modulator used in display and projection applications. MLAs offer key benefits as micro-optics as compared to the more traditional methods of beam shaping using non-diffractive optics such as lenses. RGB laser sources can be combined and shaped using a single MLA with $>90 \%$ efficiency over the area of the spatial light modulator, where a diffractive optic is designed to work with a monochromatic input and the diffraction efficiency drops as other wavelengths are 
used. Using lenslets apertures of the same aspect ratio as the spatial light modulator allows for highly efficient transformations of Gaussian input beams into to top-hat beams with rectangular spatial dimensions of $4: 3$ or 16:9 aspect ratios [18].

MLAs operate by breaking up an input beam into sub apertures and then a condensing lens behind the MLA can be used to recombine these beamlets at the back focal plane creating a uniform distribution [19]. The size of the top-hat formed at the back focal plane is controlled by the dimensions of lenslet apertures, the focal length of the MLA, and the focal length of the condensing lens [19]. Interference effects can occur that result in modulation of the actual uniformity envelope. There are two methods to reduce these coherent effects. The first one is to use a diffuser in combination with the MLA that reduces the coherence of the input laser beam, and the other way is to ensure that the size of the interference is less than the resolution of the spatial light modulator. A far-field image of the MLA illuminated under $535 \mathrm{~nm}$ light is demonstrated in Figure 1.3a and a zoomed in grayscale image capture of the MLA is shown in Figure 1.3b.

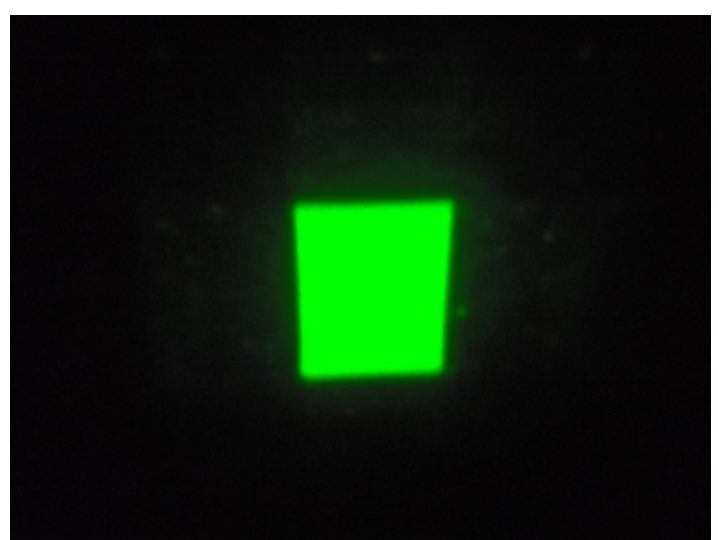

a)

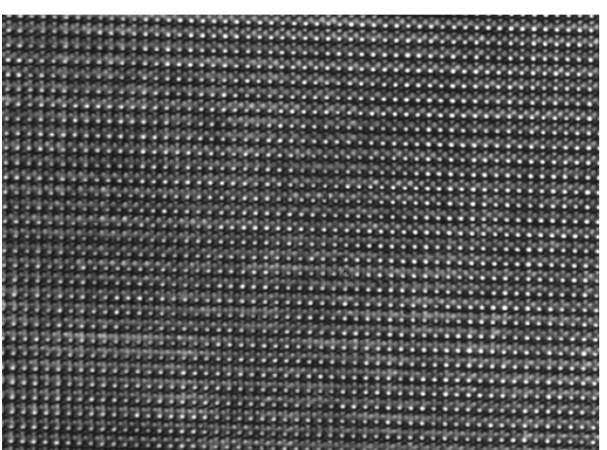

b)

Figure 1.3: MLA illuminated with $535 \mathrm{~nm}$ laser light a) full image and b) zoomed image 


\subsubsection{Polychromatic Speckle}

Current display technology relies on a three color system for additive color mixing [20]. Using red, green, and blue light sources allows for the creation of any other color needed to display the desired image or video. Pico-projector based systems can take advantage of this additive color system by utilizing three monochromatic laser sources to reproduce a full color image. The spectral response of a diffuser is often viewed as angular change in the diffractive orders. Spectroscopy systems often take advantage of the spectral response by using a diffractive grating to separate wavelengths instead of a prism. The primary concern in polychromatic diffuser design is keeping the irradiance pattern consistent across each wavelength of light that is incident on the diffuser. It is theoretically possible for angular change between two wavelengths to disrupt the projected color image. Any polychromatic diffuser design for reducing speckle should take the angular separation into account in order to avoid the addition of any visual artifacts.

\subsection{Research Outline}

The use of a rotational diffuser for laser speckle reduction is first explored to determine if varying the incident laser beam's diameter, polarization, and diffuser rotational speed will optimize temporal averaging. Two different diffuser types were utilized: binary and grayscale. Both of the diffusers are phase gratings, but each diffuser has a unique phase pattern and each diffuser relies on a different number of phase levels to accomplish speckle reduction. 
Next, secondary speckle reduction methods that can reduce speckle in conjunction with a diffuser or individually are researched. Fiber optics can be used for speckle reduction and also allow for easy beam steering which could be beneficial in a picoprojector device. Speckle contrast values are calculated for images created by a combination of diffuser and multi-mode fiber. Polarization effects on speckle are also expanded upon from previous investigations. Orthogonal polarization states will be combined prior to incidence on a diffuser to determine if theoretical speckle reduction is possible. Thorough examinations of the practicality of implementing multiple speckle techniques at the same time for a pico-projector system will be discussed.

Finally, a new diffuser design that reduces speckle contrast is proposed. Rotational diffusers excel at reducing the speckle contrast resulting from a coherent source incident on a random surface. The main drawback to rotational diffusers is incorporating a moving part into a hand-held device. Moving parts create vibrational frequencies that can cause issues. Moving parts are also a mechanical failure mode that is often undesirable with hand-held electronic devices. A dual-inline diffuser based on Hadamard matrices is designed to reduce speckle while remaining completely stationary. Variations in the Hadamard matrix pattern types reduce speckle contrast without the need for temporal averaging. Using two statistically correlated Hadamard diffusers with varying phase patterns in tangent reduces the speckle further than two generic uncorrelated binary diffusers. A Fourier-optics based modelling system was used to simulate this design. Physical manufacturing and monochromatic testing of the new diffuser was completed following design 
completion. Finally, full red, green, and blue (RGB) testing of the diffuser was completed to determine full effectiveness as a speckle reduction method for a picoprojector device. Full testing results and analysis are included. 


\section{Chapter 2: Speckle Theory}

\subsection{Complex Numbers}

A description or model is needed for laser light prior to further analysis of speckle and coherence. It is assumed the laser is fully coherent and monochromatic as well as complex in nature. Equation 2.1 demonstrates how a complex number can be broken into its real and imaginary elements [5].

$$
\underline{z}=x+j y
$$

The complex signal is $\underline{z}$ with $\mathrm{x}$ and $\mathrm{y}$ representing the real and imaginary components respectively. By a change of variables as described in Equation 2.2, a complex variable can be written in polar coordinates with $r$ being the length and $\phi$ being the angle.

$$
x=r \cos \phi, y=r \sin \phi
$$

Combining Equation 2.1 with Equation 2.2 allows $\underline{z}$ to be described by cosine and sine functions with length $r$ and angle $\phi$ as shown in Equation 2.3.

$$
\underline{z}=r(\cos \phi+j \sin \phi)
$$

Using Euler's formula [1],the cosine and sine components of Equation 2.3 can be rewritten in terms of complex exponentials as in Equation 2.4. 


$$
\underline{z}=r e^{j \phi}
$$

Equation 2.5 describes a complex harmonic wave as described by Maxwell's equation using complex exponentials from Equation 2.4 [21].

$$
\underline{A}(x, y, t)=A(x, y) e^{j \phi(x, y, t)}
$$

$A(x, y)$ is the amplitude or strength/magnitude of the wave and $\phi(x, y, t)$ is the complex exponential. The complex exponential can be broken into

$$
\underline{A}(x, y, t)=A(x, y) e^{j(\omega t)} e^{j \theta(x, y)}
$$

where $\omega$ represents the frequency of the wave moving in time, $t$, and $\theta(x, y)$ represents the phase of the wave as it moves forward in the $z$-direction. This is the preliminary model that can be used to model laser light. Since the laser is monochromatic from this point forward $e^{j w t}$ will be assumed for all individual phasor contributions and will not appear explicitly in any equations. The spatial dependency $(x, y)$ for the amplitude and phase will no longer be used. The final phasor form for the laser model is illustrated by $e^{j \theta}$ and it is assumed that the ray has some real non-unity amplitude $A$. This phasor notation can be used to show the relationship between interference and coherence that is needed to fully describe laser speckle.

Young's double slit experiment can be used to describe spatial coherence and interference principles. This experiment is pictured in Figure 2.1. A monochromatic, coherent plane wave is incident across the surface of the pinholes. Using the previous 
analysis of light we can define the incoming plane wave in phasor form by $e^{j \theta}$ once again assuming, but not explicitly describing a time component. Although both rays originally start out in phase, their path lengths change due to the difference in time it takes to travel to the same position. At point $\mathrm{P}$ on the observation plane the rays will interact with each other and the phase difference between the two rays will add negatively or positively. The destructive or constructive interference appears to the human eye in the form of fringes, as illustrated in Figure 2.2. If the number of slits in this experiment were to increase, the contributions to the interference at point $\mathrm{P}$ would increase since a larger number of differing phase components are summed together along the image plane. As the number of slits approaches infinity, the complex phasor contributions at point $\mathrm{P}$ also approach infinity, increasing the complexity of the interference. The mathematical expression for the summation of phasor contributions at point $\mathrm{P}$ is identical to the phasor contributions from speckle at any given point in a speckle image as described in Equation 2.7. 


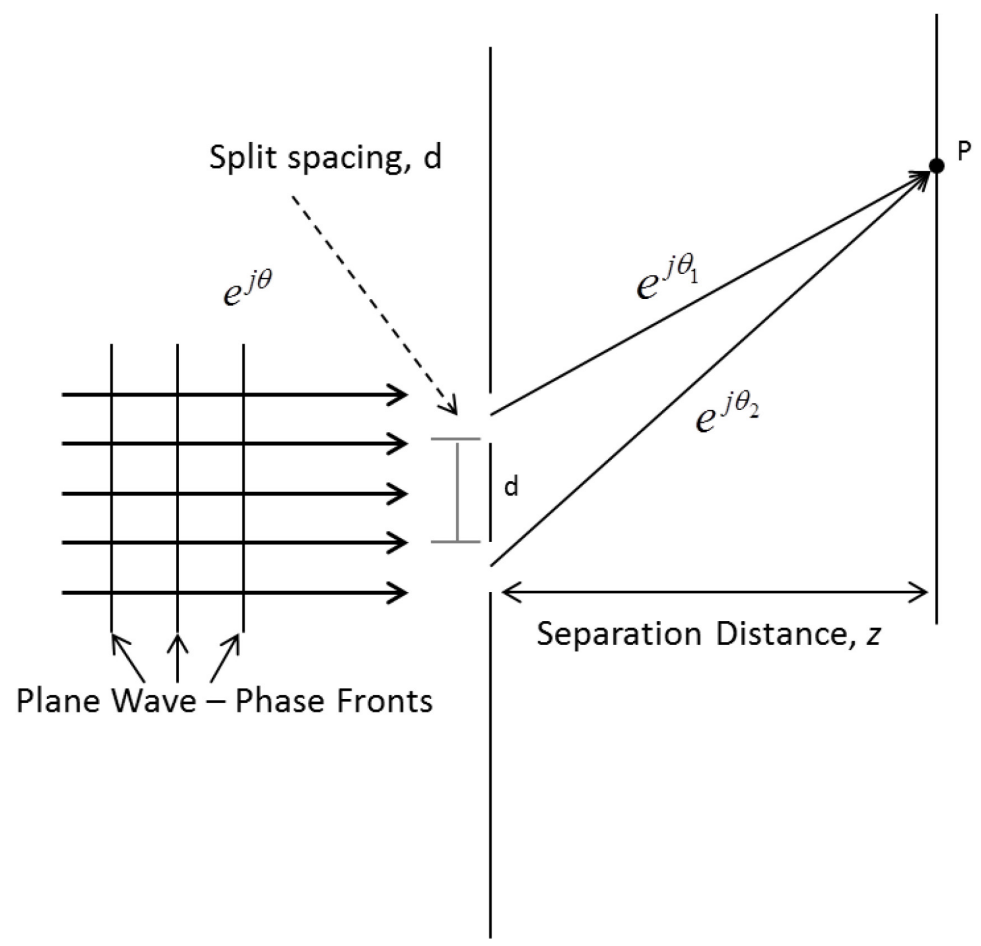

Figure 2.1: Young's double slit experiment diagram

Speckle is most commonly described as a noisy (grainy) pattern that appears when a laser spot is reflected off a "rough" surface. This roughness refers to the random surface height on the same scale as the optical wavelength incident. For visible light this is roughly accepted as $400-700 \mathrm{~nm}$, although speckle phenomena is not limited to these wavelengths. The coherent phasors of the incident light reflect and scatter from the various peaks and valleys of the illuminated surface with new random amplitudes and random phase as demonstrated in Figure 2.3. This combination produces intensity with bright or dark spots, also described as positive or negative interference similar to that previously described by Figure 2.2. 


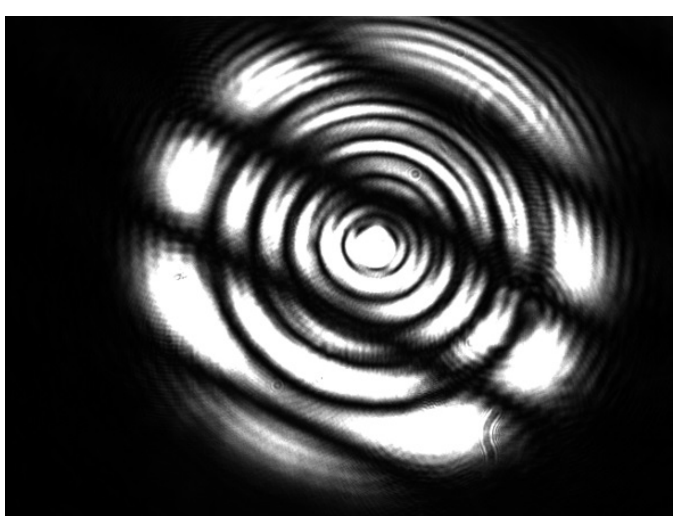

Figure 2.2: Interference fringes in circular laser beam structure

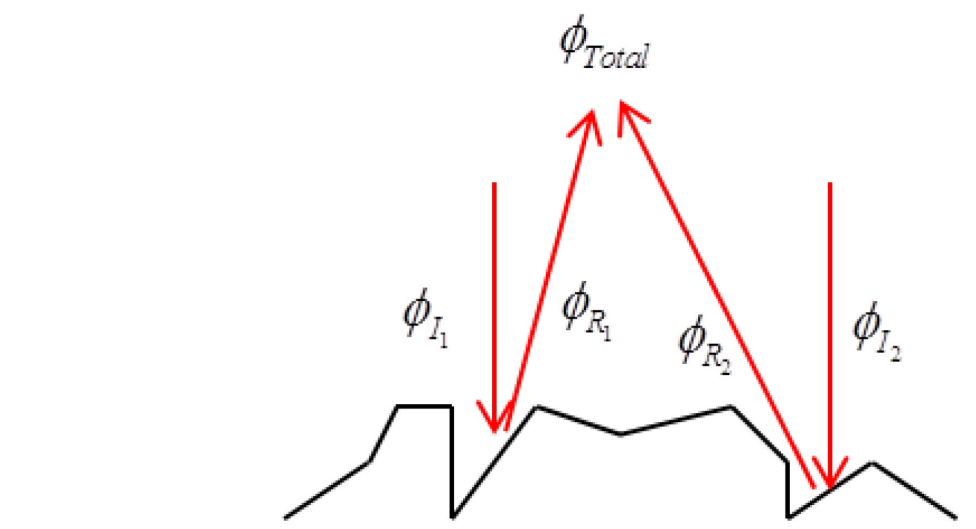

Figure 2.3: Reflection of coherent phasors from a rough surface

\subsection{Random Phasors}

Each independent contribution is complex having both amplitude and phase. Each contribution can consist of either random magnitude (length) and random phase (angle) or known length and random phase. Summations of these components result in random sums based on the relative phase of each individual and leads to constructive or destructive interference. This is demonstrated by what is known as the random walk shown in Figure 2.4. As rays (R1, R2, R3, and R4) are combined along 
the real and imaginary planes, the final ray is a summation in amplitude and phase and is solely determined by its previous contributions.

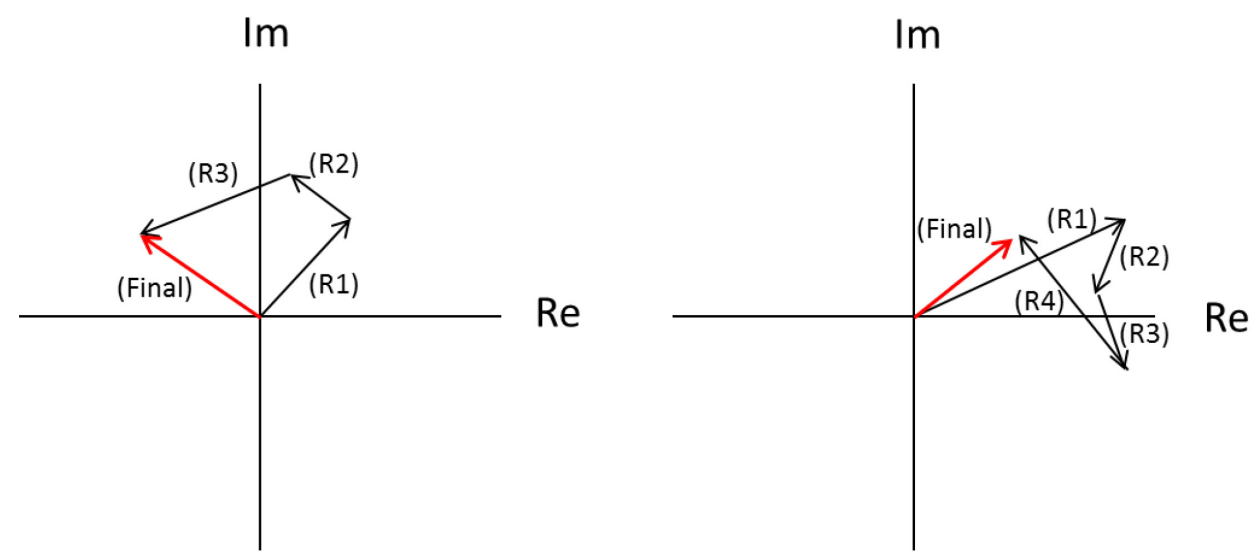

Figure 2.4: Random phasors contribute to single phasor total

The resultant phase found by a multiple ray contributions is given by Equation 2.7.

$$
A e^{j \theta}=\frac{1}{\sqrt{N}} \sum_{n=1}^{N} \underline{A}_{n}=\frac{1}{\sqrt{N}} \sum_{n=1}^{N} a_{n} e^{j \phi_{n}}
$$

$A_{n}$ is the $n$th component phasor contribution with $a_{n}$ being the individual phasor length and $\phi_{n}$ describes the direction of the phasor. The value of $\frac{1}{\sqrt{N}}$ is a scaling factor added to limit the maximum amplitude as $N$ increases towards infinity. This is necessary to represent the physical actuality of a large, but non-infinite number of phasors.

The real (Re) and imaginary (Im) components of the phasor sum given in Equation 2.7 can be separated from the complex exponential. These two components of the phasor sum are described in Equations $2.8 \& 2.9$, respectively. The real and imaginary components of the amplitude $A$ for each individual phasor contribution are 
given by a large number of random variables. As $N$, the number of contributions increases towards infinity, the probability density of the real and imaginary parts can be described by a normal distribution. This theory is known as the central limit theorem [22]. The real and imaginary distribution can be described jointly by Equation 2.10.

$$
\begin{aligned}
& R=\operatorname{Re}\left\{\underline{A} e^{j \theta}\right\}=\frac{1}{\sqrt{N}} \sum_{n=1}^{N} a_{n} \cos \phi_{n} \\
& I=\operatorname{Im}\left\{\underline{A} e^{j \theta}\right\}=\frac{1}{\sqrt{N}} \sum_{n=1}^{N} a_{n} \sin \phi_{n} \\
& p_{R, I}(R, I)=\frac{1}{2 \pi \sigma^{2}} e^{-\left\{\frac{R^{2}+I^{2}}{2 \sigma^{2}}\right\}}
\end{aligned}
$$

It is important to note that the variance, $\sigma^{2}$, in Equation 2.10 is equivalent to the variances of both the real and imaginary probabilities, $\sigma_{R}^{2}$ and $\sigma_{I}^{2}$, from Equations 2.8 and 2.9. This variance value will be used repeatedly to solve for the contrast of the speckle pattern.

It is easier to work with the resultant phasor contributions in terms of amplitude and phase, so a transformation of variables is required. The outcome of the transformation is the joint distribution of the length and angle and is given in Equation 2.11. A more detailed analysis of the transformation is found in [5].

$$
p_{A, \theta}(A, \theta)=\frac{A}{2 \pi \sigma^{2}} e^{-\frac{A^{2}}{2 \sigma^{2}}}
$$


The variance, $\sigma^{2}$, of the joint probability density function remains unchanged from Equation 2.10. Separating the amplitude and phase from Equation 2.11 can be completed by finding the marginal distribution for the amplitude, $A$, and the phase, $\theta$, respectively and is done through integration. A detailed breakdown of the integration path can be found [5]. The marginal probability density of the amplitude is described by a Rayleigh density function and is given in Equation 2.12. Similarly, the marginal distribution of the phase is given by a uniform distribution (constant valued) and is described in Equation 2.13. It is important to note that both distributions are limited to specific numeric regions.

$$
\begin{gathered}
p_{A}(A)=\int_{-\pi}^{\pi} p_{A, \theta}(A, \theta) d \theta=\frac{A}{\sigma^{2}} e^{-\frac{A}{2 \sigma^{2}}}, \quad A \geq 0 \\
\frac{1}{2 \pi}, \quad \text { for }-\pi \leq \theta \leq \pi
\end{gathered}
$$

To continue towards finding the contrast of the speckle pattern, the probability density function of the intensity is needed. A transformation of variables is completed, using Equation 2.13 to relate the amplitude with the intensity. Since the phase is uniform across a specified area, the intensity distribution is not affected by theta. The intensity distribution is described by Equation 2.14.

$$
I=|A|^{2}
$$




$$
\begin{aligned}
& p_{I}(I)=p_{A}(\sqrt{I})\left|\frac{d A}{d I}\right|=\frac{1}{2 \sqrt{I}} p_{A}(\sqrt{I}) \\
& p_{I}(I)=\frac{1}{2 \sigma^{2}} e^{-\frac{I}{2 \sigma^{2}}}, \quad I \geq 0
\end{aligned}
$$

Remember that $\sigma^{2}$ is the variance of both the real and imaginary elements of the amplitude and phase. The probability density function for the intensity takes the form of a negative exponential function. This distribution is classified as a negative exponential distribution. Once again the region of existence is limited and is determined by the region of existence for the amplitude, which cannot be negatively valued. The first moment (average) of the intensity is found by the expectation (direct integration) of $I$ as described in Equation 2.14.

$$
E[I]=\int_{-\infty}^{\infty} I \cdot \frac{1}{2 \sigma^{2}} e^{-\frac{I}{2 \sigma^{2}}} d I=2 \sigma^{2}
$$

The $2^{\text {nd }}$ moment, variance, of the a negative exponential distribution can described as the square of the $1^{\text {st }}$ moment [22] which leads to a standard deviation of the intensity as described in Equation 2.16.

$$
\operatorname{Var}=(I)^{2}=\left(2 \sigma^{2}\right)^{2}
$$

The contrast of the speckle pattern can now be found through the use of Equation 1.1, giving a contrast value of unity for our speckle pattern. This is referred to as fully developed speckle and is the ideal case of any speckle pattern. As the phase of the contributing phasors becomes non-uniform the contrast decreases. Thus the contrast 
can be described as a region of interest of $0<\mathrm{C}<1$. While the ideal case of speckle is unity, it is not often seen under imaging situations due to air particle interference of the laser pattern. It is conjectured that speckle contrast of $<0.05$ is needed to sufficiently reduce eye irritation and headaches caused by long exposure to laser illuminated images [23]. There is some debate regarding the threshold of the speckle contrast for a projector system and researches have proposed contrast values of well below 0.02 may be required [17]. A recent experiment was conducted at the University of Brussels to compare speckle contrast values versus human eye perception to verify the speckle contrast threshold value [24]. The percentage of subjects identifying speckle in each image varied drastically between green, red, and blue colored speckle images. There were still viewers who were not able to confirm the existence of speckle in the image even with speckle contrast as low as 0.03 [24]. Although the data from this experiment clearly shows the need for very low speckle contrast values, large, bulky or complex speckle reduction techniques are not practically able to be integrated into pico-projector systems. The following chapters detail the investigation of a speckle reduction solution for the specific application of pico-projector devices. 


\section{Chapter 3: Diffractive Diffusers}

\subsection{Rotational Diffuser Theory}

A commonly used speckle reduction technique is the temporal averaging of a fabricated intensity pattern using a diffuser [25]. The averaging occurs from rotation or vibration of the diffractive element. The diffuser structure can vary and multiple optical designs have been used to successfully lower the contrast of a monochromatic speckle pattern [3]. A stationary diffuser introduces a single independent phase change. By moving the diffuser, $K$ independent realizations of the diffuser are averaged together. When rotating occurs, the number of patterns averaged determines the speckle reduction provided by the diffuser. The contrast from $K$ patterns is determined by Equation 3.1 [3].

$$
C=\sqrt{\frac{1}{K}}
$$

The ideal situation involves $K$ approaching infinity, resulting in an indefinitely small speckle contrast. Since the eye samples at $23 \mathrm{~Hz}[10]$ in order for temporal averaging to completely negate speckle, a diffuser would need to rotate a number of times approaching infinity, all within 0.05 seconds. Speckle contrast for each captured image was found by expanding Equation 1.1 as described in Equation 3.2. 


$$
C=\frac{\sqrt{\left\langle I^{2}\right\rangle-\langle I\rangle^{2}}}{\langle I\rangle}=\frac{\sqrt{\frac{\sum_{i=1}^{N}\left(I_{i}-\langle I\rangle\right)^{2}}{N-1}}}{\langle I\rangle}
$$

Testing was completed to experimentally determine the maximum speckle contrast reduction accomplished by rotating diffusers. For this experiment two types of diffusers were utilized; a grayscale and a binary scale. The grayscale diffuser uses 64 phase patterns to create a pseudo random phase distribution. This creates a more continuous profile along the edge of the diffuser. In contrast, the binary diffuser only has 2 phase levels. This creates an edge contrast between the light and dark sections of the image due to the Fresnel diffraction of the sharp edges of the diffuser. Both were designed with the intent of destroying the coherence of laser sources to decrease the modulation effect due to periodicity of a micro-lens array (MLA) like those described in Section 1.2.2. The images in Figure 3.1 that follow illustrate the separate speckle patterns created by both types of diffuser. Figure 3.2 shows the dimensions of the diffusers.

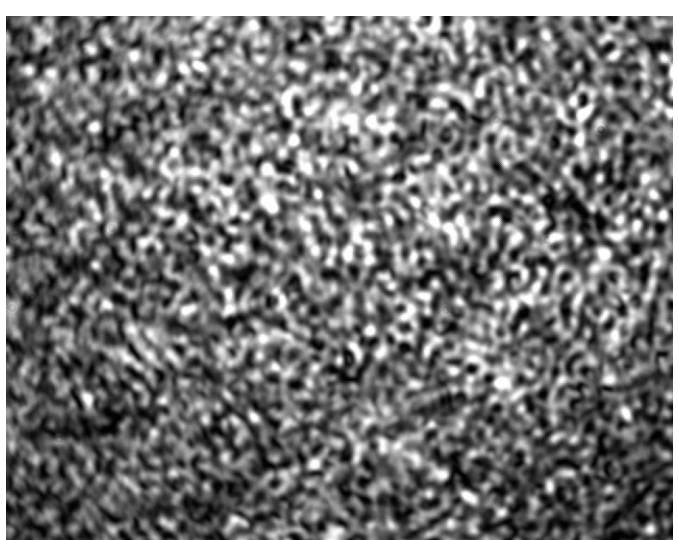

a)

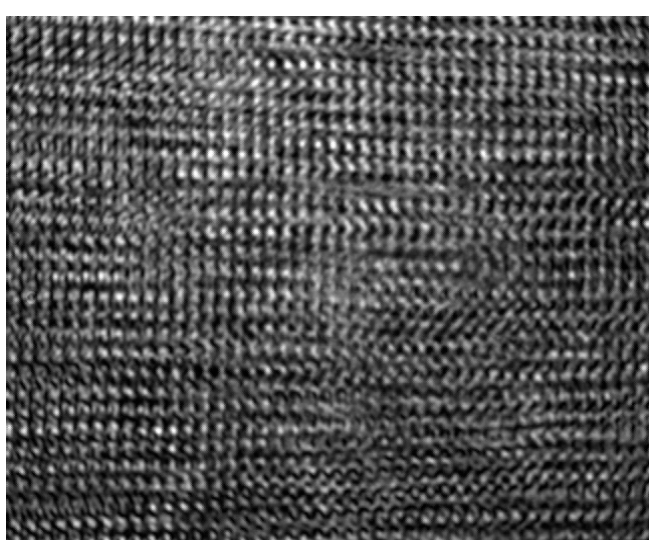

b)

Figure 3.1: Speckle Image caused by laser incident on a) grayscale diffuser b) binary diffuser 


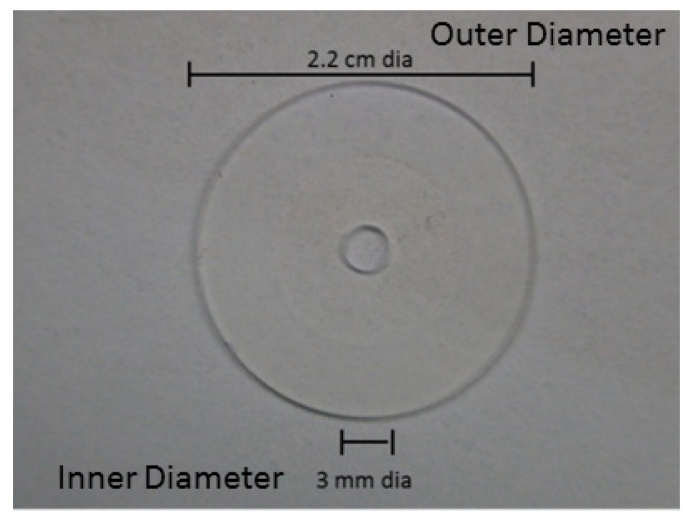

Figure 3.2: Diffuser image with metric dimensions

Speckle contrast from a rotating diffuser is determined by number of realizations averaged across the detector. Increasing the diameter of the incident laser beam should hypothetically result in a lower speckle contrast value due to the increase in illuminated phase pattern. Laser beam size is varied across the surface of the diffuser to determine the relationship between laser beam size and speckle contrast. The size of the illuminated spot on the diffuser will also be measured versus rotation speed of the diffuser itself. Rotation speeds will vary between stationary up to 9 times the integration time of the eye $(23 \mathrm{~Hz})[10]$. The final investigation is varying the polarization state between linear and non-linear against varying rotational speeds of the diffuser. Rotational speeds, again, include stationary up to 175 rotations per second.

\subsection{Measurement Setup}

In order to verify the design of the diffusers, images of the stationary speckle pattern from the diffuser are compared against images taken of the same diffuser spinning. Equation 3.2 is used for all contrast measurements and calculations, normalizing for 
proper variance measurements. A Thorlabs' DC210 black and white, firewire digital camera was used for image capture. Maximum speed of image capture is $30 \mathrm{~Hz}$ with variable aperture and gain values. The gain was set to $9.91 \mathrm{~dB}$, the exposure was set to 426 (unitless multiplyer) and the shutter speed was set to $12.12 \mathrm{~ms}$ for the duration of the experiments. The camera contains a CCD array of $640 \times 480$ with a pixel size of $7 \mu \mathrm{m}$. Throughout the measurements the speckle spot size $\rho$ (which is related to the wavelength $\lambda$, the distance $\mathrm{D}$, and the beam diameter $\mathrm{d}$ ) given by

$$
\rho=\frac{1.22 \lambda D}{d}
$$

was kept constant at approximately $64 \mu \mathrm{m}$. Keeping the speckle spot size constant will cancel out any discrepancies in measurement caused by random speckle bright spots in the images [26].

Two separate monochromatic lasers with optical wavelengths of $532 \mathrm{~nm}$ and 632.8 $\mathrm{nm}$ (green and red respectively) were used in the experiments. The $532 \mathrm{~nm}$ laser was a diode pumped solid-state (DPSS) laser purchased from Snake Creek Lasers [27]. The $632.8 \mathrm{~nm}$ laser was a Helium Neon (HeNe) purchased from Melles Griot [28]. The irradiance of the laser sources prior to any optical element were $10 \mathrm{~mW}$ and 30 $\mathrm{mW}$ for green and red, respectively. The DPSS $532 \mathrm{~nm}$ laser had pseudo-random polarization, while the HeNe laser had strictly linear polarization. To avoid impacting the speckle contrast for beam diameter measurements both lasers were passed through a linear polarizer (aligned perpendicular to the optical bench) to equalize polarizations between both laser sources. The pseudo-random polarization of the 
DPSS laser will be used to measure differences in speckle contrast from a rotating diffuser with linear polarization (via polarizer) versus a rotating diffuser with random polarization. In this situation random polarization is defined as having multiple linear polarization states from the same laser source. Images of the laser setup in use are shown in Figure 3.3a with the diffuser mount pictured in Figure 3.3b. The speckle contrast for both stationary and rotational instances of the diffusers was calculated using Equation 3.2.

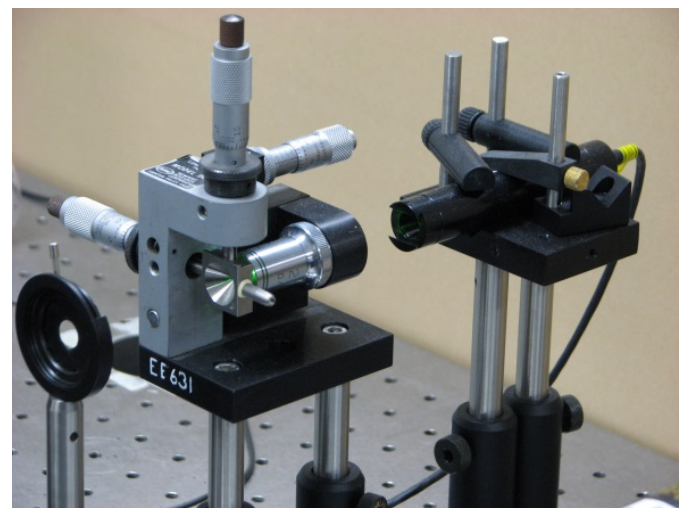

a)

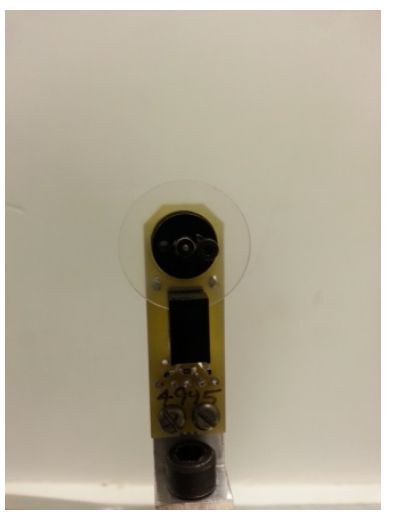

b)

Figure 3.3: a) Optical bench top layout and b) diffuser rotational mount

Three beam sizes were chosen: $4.6 \mathrm{~mm}, 2.99 \mathrm{~mm}$, and $1.6 \mathrm{~mm}$. The $4.6 \mathrm{~mm}$ beam size was chosen because it the largest beam size that can fit in the window of the diffuser. The smallest beam diameter is the smallest that can be established using the equipment available. The middle beam size $(2.99 \mathrm{~mm})$ was chosen to be approx. halfway between both the large and small beam size for variability. For the entire experiment the laser's power intensity before image capture was kept steady, using neutral density (ND) filters, at $0.1 \mu \mathrm{W}$ with $+/-10 \%$ accuracy. The experimental optical bench layout used is shown in Figure 3.4. For all experimentation, collimated light must be projected onto the face of the diffuser for it to operate as designed. The 
orders formed from the diffusers were distinct for each diffuser type and the separation of orders was based on the beam size incident on the face of the diffuser.

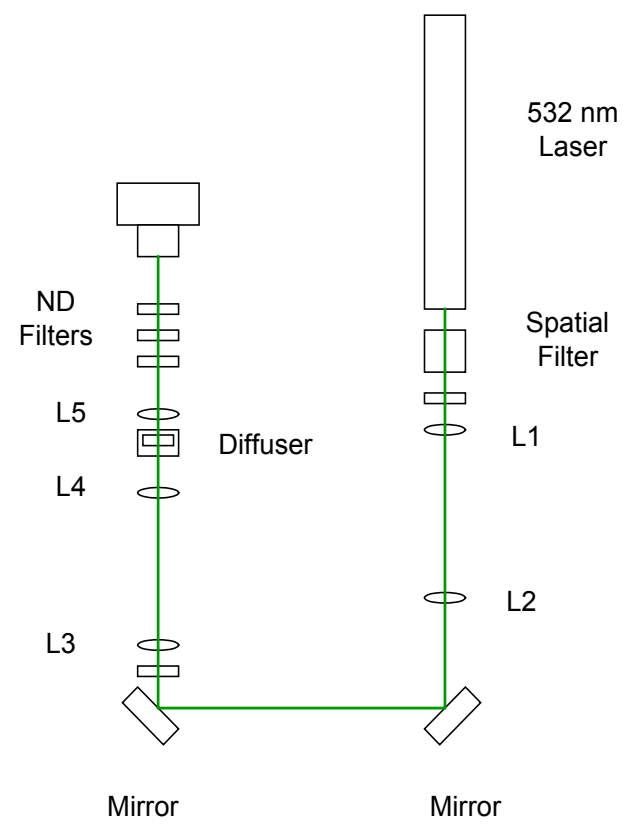

Figure 3.4: Rotation diffuser experimental setup layout

The laser is spatially filtered, collimated, and then manipulated to the proper beam diameter for the analysis prior to incidence on the diffuser surface. Once the beam has passed through the diffuser a lens, L5, is used to expand/contract the central diffractive order (caused by the diffuser) to ensure the order size matching the CCD array size of the imager. L5 will vary because the beam size variations incident on the diffuser will create different central order diameters and must be manipulated accordingly to match the $\mathrm{CCD}$ array.

The sampling rate of the imager was set to 30 frames per second to approximate the sampling rate of the human eye. The camera integration rate was adequate for measuring speckle contrast for rotating diffusers since the goal in speckle reduction is 
to increase the visual clarity of the image for the human eye. The diffuser was attached to a rotational chopper motor as shown in Figure 3.3b. Changing a constant input voltage caused the motor to rotate faster or slower. The rate of rotation for the diffuser was varied from $30 \mathrm{~Hz}$ to $175 \mathrm{~Hz}$ where $175 \mathrm{~Hz}$ is the highest available rotational speed from the chopper motor.

\subsection{Speckle Contrast Results}

Figures $3.5,3.6,3.7$, and 3.8 show comparisons of beam diameters for speckle contrast versus diffuser rotation speed. Both laser wavelength results and data are illustrated in the graphs.

Comparison of Beam Sizes using 07-133-02 Grayscale Diffuser (632 nm)

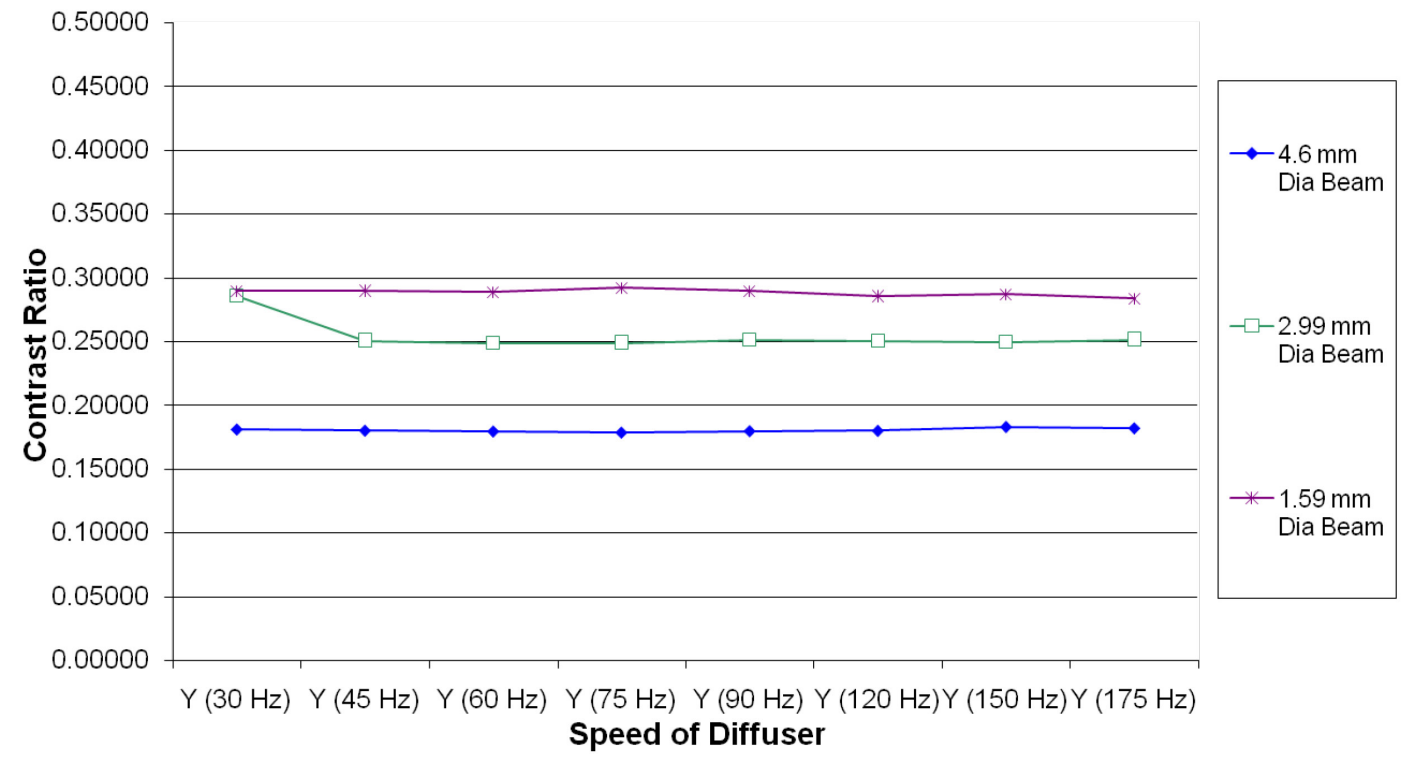

Figure 3.5: Comparison of Beam Sizes using 07-133-02 Grayscale Diffuser (at 532nm) 


\section{Comparison of Beam Sizes using 07-096-01 Binary Diffuser (632 nm)}

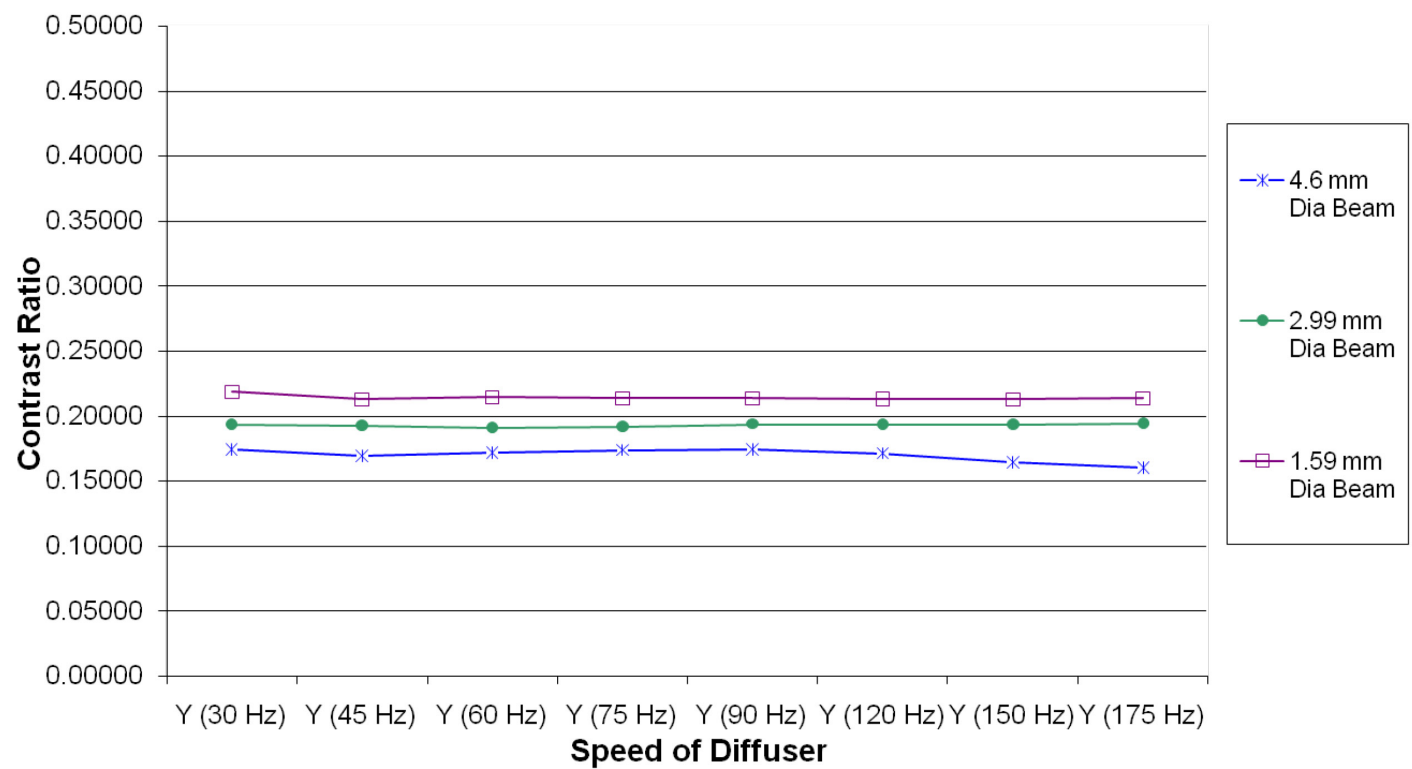

Figure 3.6: Comparison of Beam Sizes using 07-096-01 Binary Diffuser (at $632 \mathrm{~nm})$

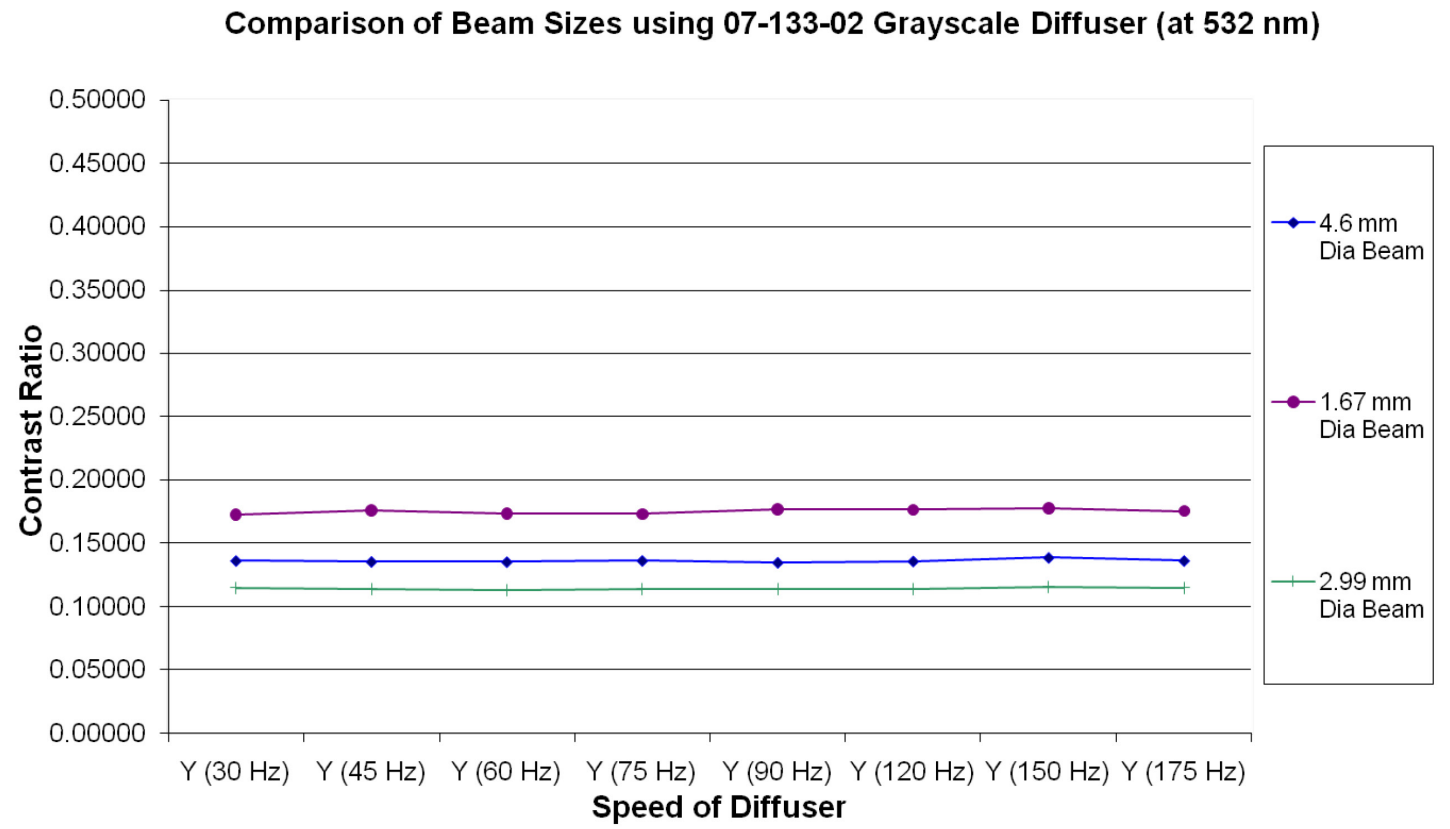

Figure 3.7: Comparison of Beam Sizes using 07-133-02 Grayscale Diffuser (532nm) 
Comparison of Beam Sizes using 07-096-01 Binary Diffuser (532 nm)

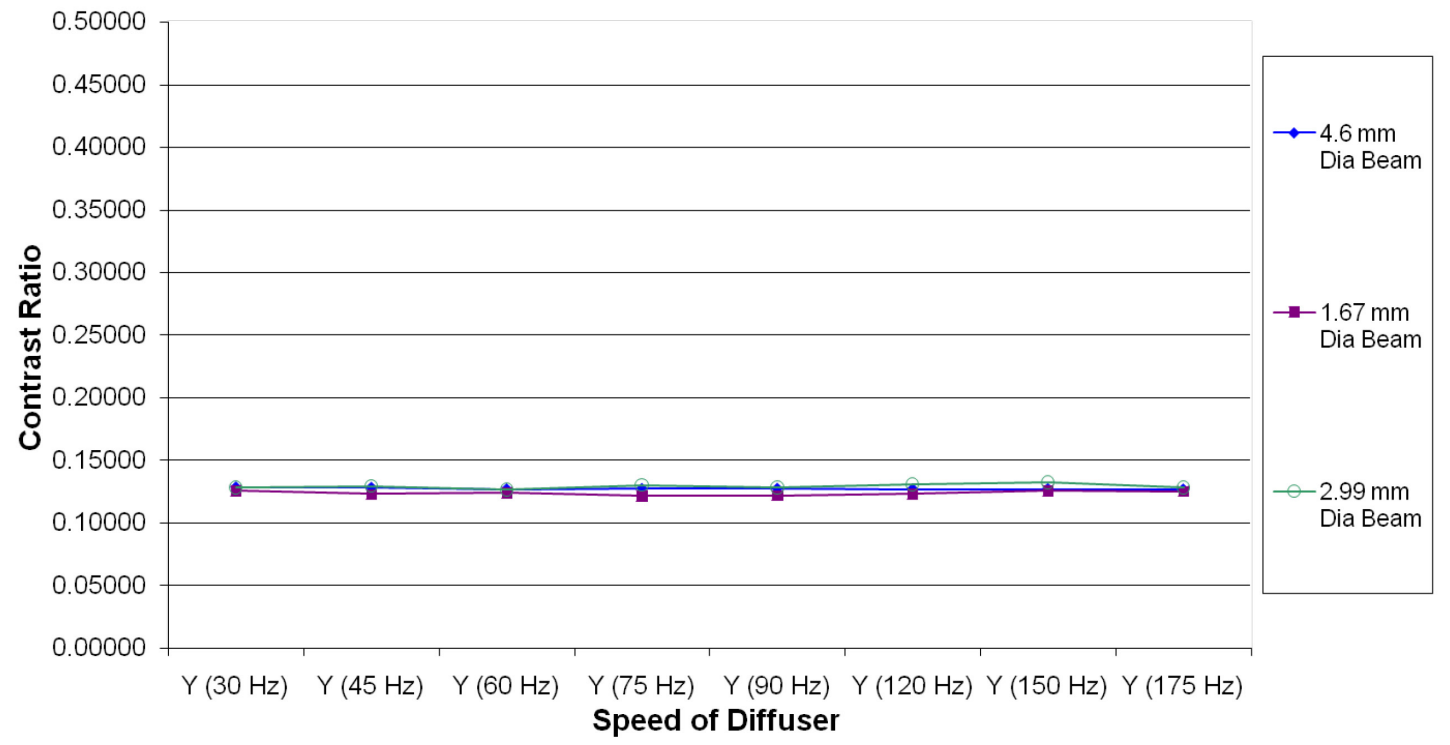

Figure 3.8: Comparison of Beam Sizes using 07-096-01 Binary Diffuser (532nm)

Each of the graphs represents a different wavelength with one type of diffuser. All three beam diameters are represented, as well as the speed at which the diffuser rotates for comparison purposes. The diffuser speed does not appear to affect the performance of either type of the diffuser; taking into account an approximate $10 \%$ measurement error due to power level fluctuations. In addition, the beam size does slightly affect the reduction of the contrast, but does not do so consistently. In fact all the contrast measurements are within a $10 \%$ margin that once again falls into the overall measurement error percentage. Although it is shown that the diffusers did operate more efficiently with incidence of the $532 \mathrm{~nm}$ wavelength beam than with the $632.8 \mathrm{~nm}$ wavelength beam; yet the difference between the performance still falls within the measurement error allotted. 
Making use of polarization states in order to affect the performance of the diffusers in reducing the contrast ratio was also investigated [29]. The $632.8 \mathrm{~nm}$ laser had a polarization ratio of greater than 500:1 making it very linearly polarized. The $532 \mathrm{~nm}$ green laser was, in contrast, randomly polarized with a ratio of 5:1. In order to separate polarization states, linear polarizers with 10,000:1 extinction ratios were used within the setup for the $532 \mathrm{~nm}$ beam. The previous experiment set involving three beam sizes on the diffusers was repeated for the $532 \mathrm{~nm}$ linearly polarized beam. The following graphs (Figures $3.9 \& 3.10$ ) detail the comparison between speckle contrast ratios for vertical and random polarization states for the green laser. Once again, multiple beam diameters were compared to determine if their affects compounded with polarization diversity.

Comparison of Polarization States for Varying Beam Sizes and Speeds using 07-09601 Binary Diffuser $(532 \mathrm{~nm})$

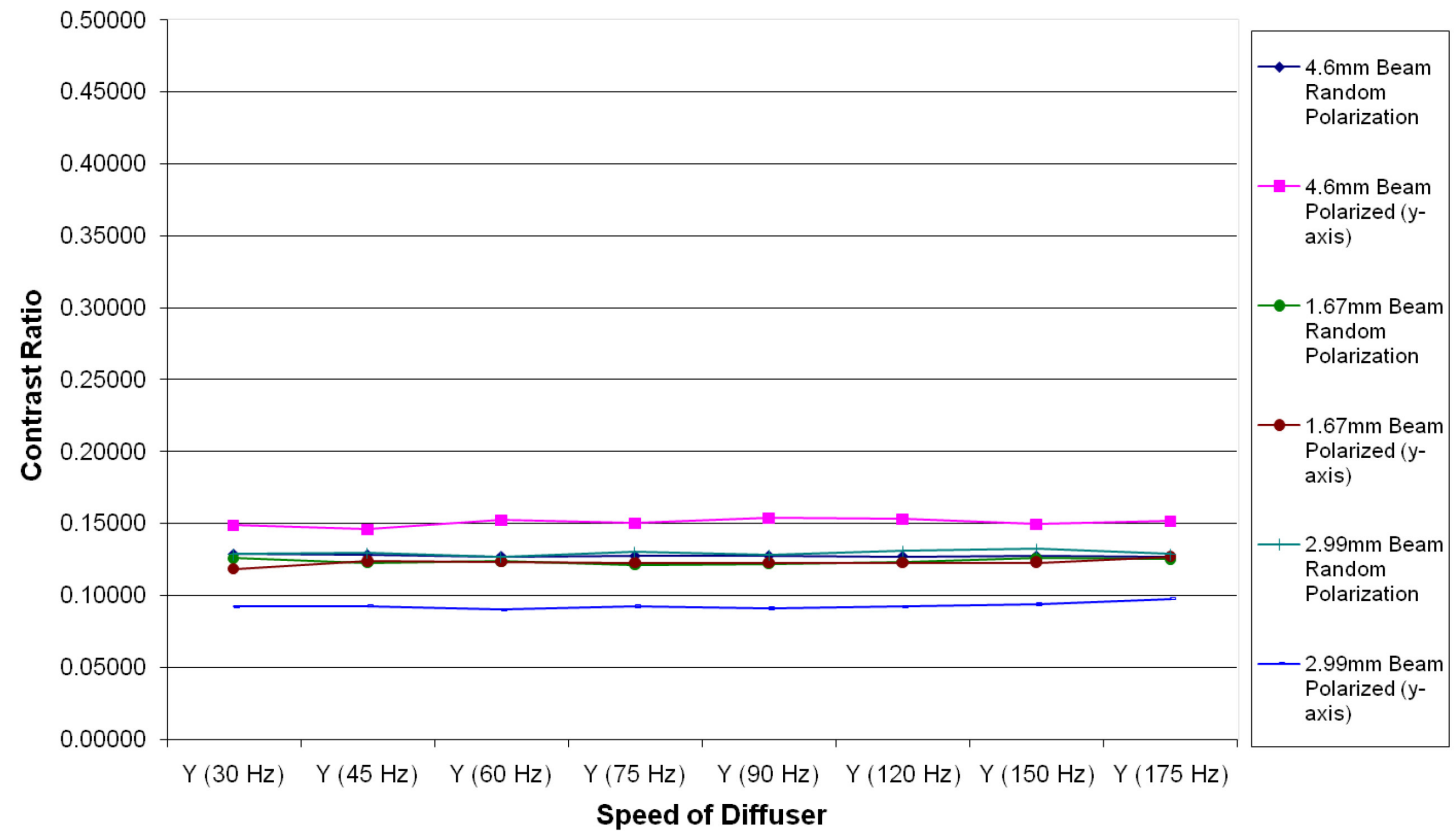

Figure 3.9: Comparison of Polarization States for Varying Beam Sizes and Speeds using 07-096-01 Binary Diffuser (532nm) 


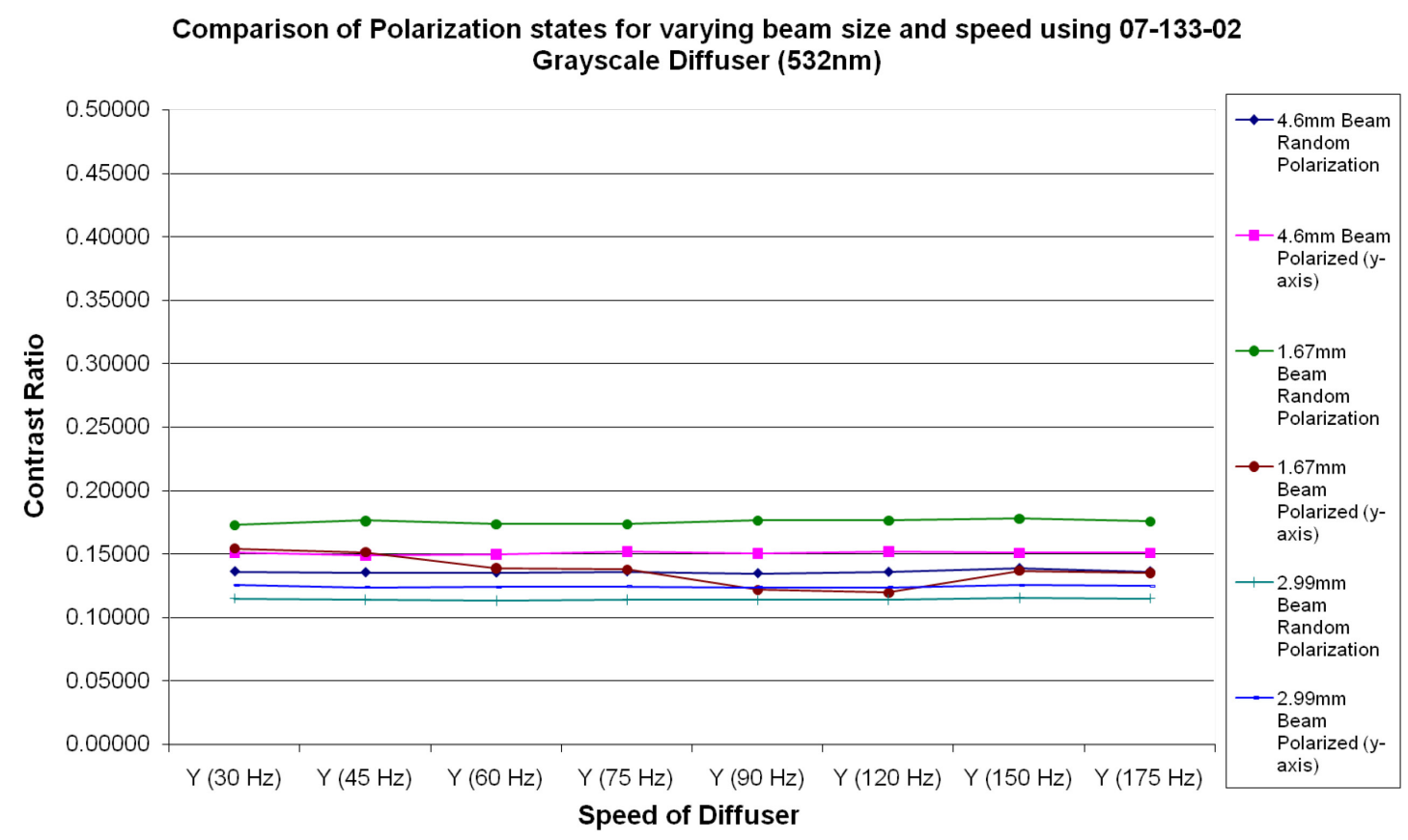

Figure 3.10: Comparison of Polarization states for varying beam size and speed using 07-133-02 Grayscale Diffuser (532nm)

Once again it is seen that the speed of the diffuser does not directly affect operability and performance. There is also no change that can be seen between varying polarization states for the contrast. In addition, the beam diameter once again does not make a drastic impact on the speckle contrast ratio taking into account processing/calculating error. For reference, Figure 3.11 shows the comparison between the stationary speckle image versus the rotating speckle image. 


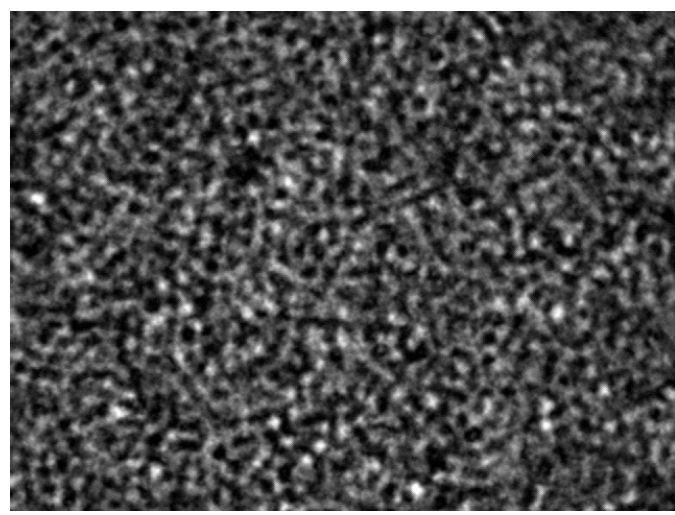

a)

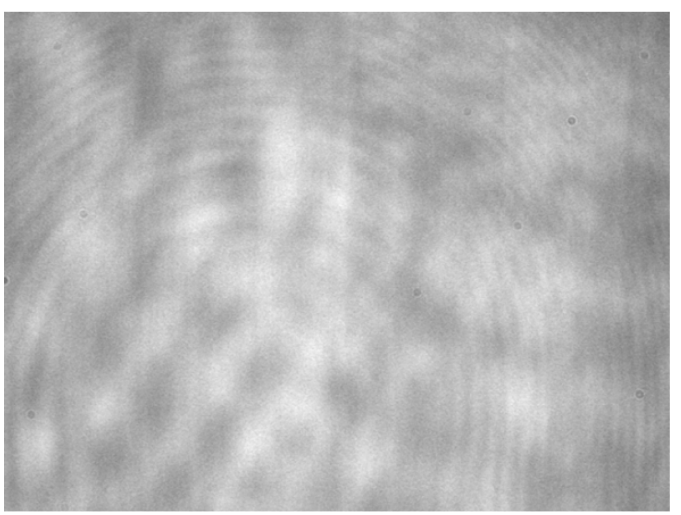

b)

Figure 3.11: a) Stationary speckle (grayscale) image and b) Rotational diffuser speckle image (at $90 \mathrm{~Hz}$ )

Lastly, diagonal cross sections of the intensity for the images were extracted. The following figures show the variance difference between images with a rotating diffuser to that of one using a diffuser to create a noisy speckle. Both types of diffusers are illustrated. The variance number for the grayscale image with speckle is 4.18 while the variance between pixel intensities with the rotating diffuser is 2.19 . The variance number for the binary image with speckle is higher compared to the grayscale at 6.55 , while the variance of the rotating binary diffuser is 2.21 . The variance of the intensity is reduced by the diffusers. 


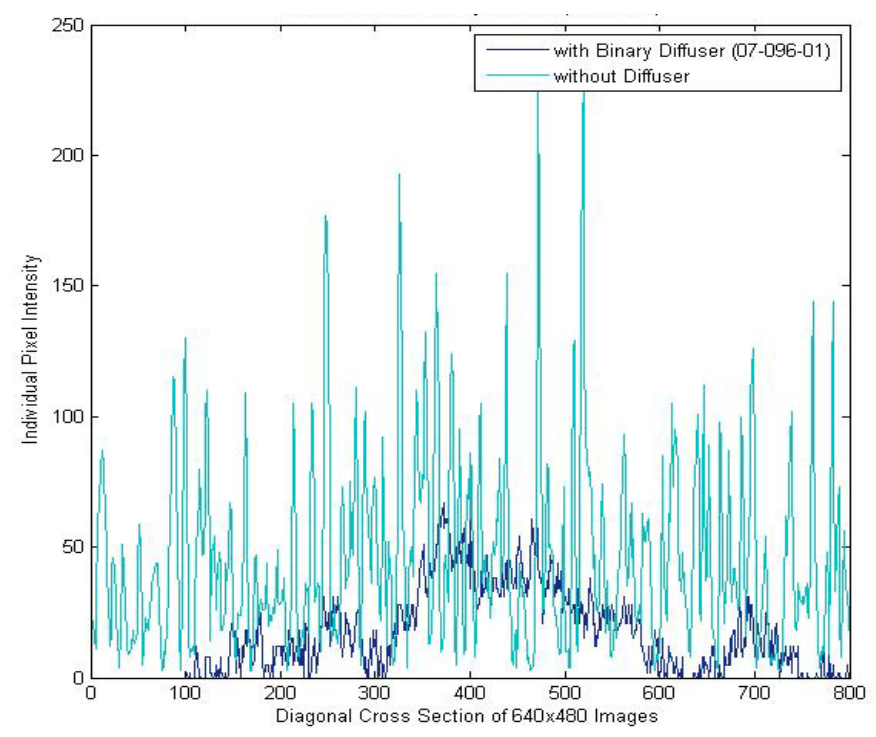

a)

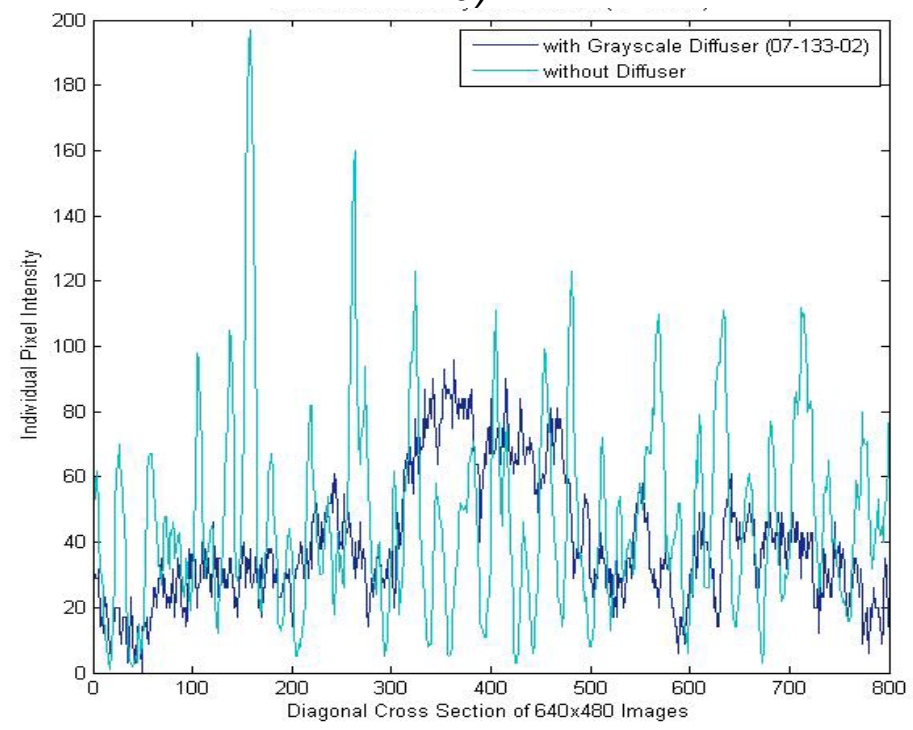

b)

Figure 3.12: Diagonal cross section of speckled images (Beam $2.99 \mathrm{~mm}$ ) with and without a) binary diffuser (07-096-01); b) grayscale diffuser (07-133-02)

\subsection{Rotating Diffuser Conclusion}

The experimental results show that the beam size and polarization do not drastically affect the operability of the diffusers both when rotating and when stationary. Although the diffusers do provide some practical problems due to the multiple 
diffractive orders, they do offer the ability to create a more aesthetically pleasing image for pico-projector devices. The diffuser types also give insight into future investigations for usage, such as spatial averaging using polarization diversity. Using multiple diffusers or multiple beams passed through the same diffuser could allow for multiple-image averaging to occur and thus reduce the speckle contrast even more. Further investigations to analyze alternative speckle reduction techniques using diffusers or more static coherence manipulative devices are required. Testing is also needed using blue light to show a full RGB speckle reduction element is possible for pico-projector systems. 


\section{Chapter 4: Secondary Speckle Reduction Methods}

\subsection{Introduction to Laser Diodes and Fiber Optics}

The previous work demonstrated using diffusers to reduce speckle from lasers at wavelengths of $532 \mathrm{~nm}$ and $632.8 \mathrm{~nm}$. Full image projection requires RGB input, so the next step was to test using a blue diode, $(450 \mathrm{~nm})$. However, this diode suffers laser structure issues that standard optical elements are not able to solve. Basic double heterostructure lasers will yield a distinctive elliptical, fast expanding beam shape. This wave front requires specialized diffractive optics to negotiate into a collimated, circularized beam. A collimated, circularized beam is preferred when utilizing diffusers for speckle reduction activities as this allows for optimum power to be put into the lowest order modes. Multiple techniques have been investigated including using cylindrical lenses, curved mirrors, and even specialized gradient-index (GRIN) lenses and will be investigated in combination with rotational diffusers.

A common approach to creating a circular beam from an elliptical beam is to use two cylindrical lenses to collimate each axis of the wave front separately. Generally, noncircular beams have a fast axis (long) and slow axis (shorter width) creating the elliptical shape [30]. A strong cylindrical lens first collimates the fast axis followed by a weaker lens to collimate the slow axis creating a circular beam. The alignment and proximity to the diode point source is essential in creating the proper beam. An issue that can arise is that the laser diode "cans" may be built in a way to not allow 
the first lens close enough to the diode in order to capture the full amount of light. While this method is still possible if the diode is removed from the canister, this creates more problems with having to recreate housing with power and heat management setups; instead, by keeping the main diode cylinder less manipulation is needed and diodes can be added to a setup with ease when needed.

Another possible optical scheme involves using specialized optics, such as GRIN or ball lenses [31]. GRIN lenses are rod shaped lenses that use gradual changes in the refractive index to shape and focus light. GRIN lenses are often used to collimate light from the output of a fiber optic creating a small usable beam. Using the same basic theory, these rod lenses can be butted up against the case of a diode to collimate the light. The issue becomes having a strong enough GRIN lens to overcome the expansion of the laser beam. In this case, a lens with the right thickness could not be used because proper usage requires placement of the GRIN lens at a closer distance to the diode than can be physically achieved. Ball lenses are primarily used to couple fibers with detectors or other optical components. The unique curvature of a ball lens allows full collimation given all the light is incident onto the lens' surface. The downside is similar to the GRIN lens in that it needs to be very close to the initial expansion point of the laser diode structure. In addition, standard (non-custom) ball lenses are generally not able to overcome larger rates of expansion.

A length of optical fiber would provide the same type of reforming that a GRIN lens could display; circularizing the elliptical beam shape from the wave bouncing around within the fiber. A single mode fiber would be the most obvious choice because of the 
low amount of interference noise. Unfortunately, for a $450 \mathrm{~nm}$ wavelength laser diode, a standard single mode fiber would have to have a core diameter of around $0.93 \mu \mathrm{m}$. The fiber optics core requirement can be calculated with Equation 4.1 [32] shown below by having the value of $\mathrm{V}$ (normalized frequency) be 2.405 and solving for diameter $d$. NA represents the numerical aperture (defined here as 0.37 ) and $\lambda$ is wavelength. A fiber of this size is very difficult to utilize because so little power can be propagated. This power loss occurs mostly due to coupling, although significant power loss can occur from high modes being radiated out of the fiber should extreme bending transpire. As previously discussed, the structure of the laser diode standard focusing is quite difficult with astigmatic beams and thus the amount of power to be concentrated into the fiber is already highly limited.

$$
V=\frac{2 \pi}{\lambda}\left(\frac{d}{2}\right) \times(N A)
$$

Supplementary literature shows research has been completed using multi-mode fiber optics to reduce speckle [16]. By vibrating the fiber, mode mixing occurs at the output causing a reduction in the coherence prior to imaging. This can create a reduction from $20-30 \%$ in the speckle contrast measurements [16]. A multi-mode fiber can reform the beam in the same way a single mode would, thus meeting the needed criterion. In addition, fiber core diameter requirements at $450 \mathrm{~nm}$ can now be inverted allowing the use of the largest fiber available. This will allow for the most power available to be propagated through the fiber optic primarily through high coupling efficiency. The main drawback of using such a large fiber is its stiffness and 
thus high bend radius. Manipulation of the incident laser beam by passing through a fiber is investigated to determine whether using fiber optics for beam steering in projectors is viable [33]. Finally, an investigation into polarization manipulation and its effects on diffuser speckle contrast output is conducted and analyzed.

\subsection{Speckle Contrast Results}

Part of the investigation was experimenting with various lengths of the same fiber to determine whether or not length will play a part in the circularization of the laser and to determine the effect length of fiber has on speckle contrast. In addition, different optical fiber lengths may have a definitive impact on the power output, which may be more of a deciding factor in using fiber optics for pico-projector devices. Starting fiber lengths of $100 \mathrm{~cm}, 50 \mathrm{~cm}$, and $25 \mathrm{~cm}$ were chosen and tested. The $100 \mathrm{~cm}$ length provided $19 \mathrm{~mW}$ of output power and had a very circular, even beam shape. The 50 $\mathrm{cm}$ fiber piece outputted $14 \mathrm{~mW}$ of power and had a circular beam, but with a few disruptive artifacts. Finally, the $25 \mathrm{~cm}$ fiber provided a $15 \mathrm{~mW}$ output power with an adequate circular beam shape. The discrepancy with the $50 \mathrm{~cm}$ fiber may be due to an imperfectly polished fiber end, yet with the $25 \mathrm{~cm}$ performing so well, it was unnecessary to continue experimentation with the longer fiber pieces.

As previously mentioned, the propagated beam must be collimated prior to incidence onto the diffusers in order to reduce excess diffractive modes. The problem is that the wave front from multi-mode fiber optics are difficult - if not impossible - to collimate with just a single lens [15]. A focused image can be developed, but not a stable constant beam. Attempts to use multiple lenses to collimate the after image of 
the fiber were unsuccessful. Spatially filtering the beam from the output of the fiber was then tried. Although this worked successfully, it eliminated the benefits of the fiber as well as reduced the power level down to almost miniscule proportions. However, it gave way to an interesting idea; using just the pinhole and a lens to create a collimated beam. The pinhole would eliminate a large number of modes as an aperture and would ensure the modes exiting the fiber were from the central region of the core where, based on fiber optic ray tracing, would be more slowly expanding [1]. The largest pinhole size available, $100 \mathrm{um}$, was placed directly behind the output face of the optical fiber. The power loss was roughly $99.3 \%$. This is rather high for practical applications, but at this point it suffices for the investigation and provides a collimated beam to be used for the diffuser experiment.

After collimation, the beam was incident on the diffuser surface prior to projection onto a detector. As shown in Section 3.3, beam size does not affect speckle reduction from the diffuser so long as the entire beam is fully propagated onto the diffuser [34]. The projection lens after the diffuser was determined based on the speckle size from the diffuser. This was set to maintain approximately constant speckle spot size onto the detector of roughly $8 \mu \mathrm{m}$ diameter. The final image displays compounded speckle, or speckled speckle. The smallest speckle is caused by the mode structure of the optical fiber being used for circularization. This fiber speckle will shift in space over time given physical disruptions to the fiber or coupling setup. This can cause blurring and thus loss of signal strength which means data loss for communications systems, but time-averaging will decrease the speckle contrast. The number of 
speckles produced by the fiber, within the far-field can be roughly determined by Equation 4.2 [15]. While this information is useful in giving a rough approximation it is only for understanding purposes as the pinhole limits the number of modes allowed through the output of the fiber.

$$
N_{\text {speckles }} \cong 2\left(\frac{r_{0} \times N A}{\lambda}\right)^{2}
$$

In this equation $r_{0}$ is the radius of the fiber core with $N A$ and $\lambda$ the same as for the previous equation.

A procedure for measuring the amount of vibration or movement of the fiber in relation to the time-average blurring effect of the speckle was used for reference [16]. This investigation focuses on the reduction of speckle through the use of the diffuser in relation to the fiber's compounded speckle. The physical shift of the fiber is eliminated through the use of a "floating" optical environment. This provides a static speckle from the optical fiber, which is then incident onto the face of the diffuser. Figure 4.1a shows the final image from the stationary binary diffuser. 


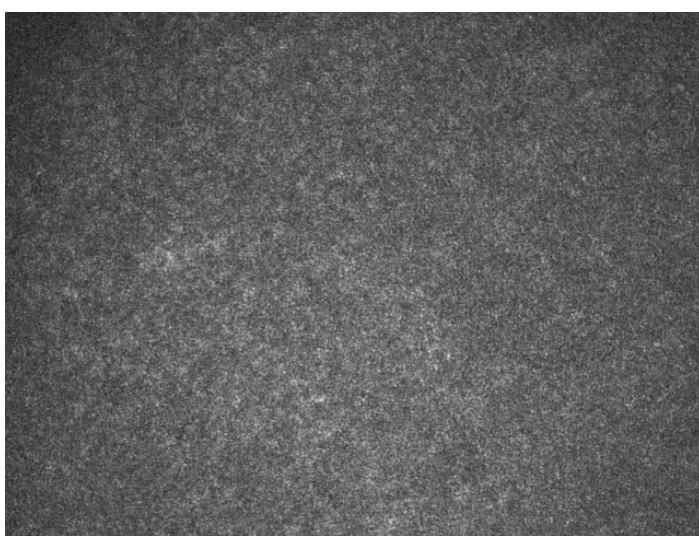

a)

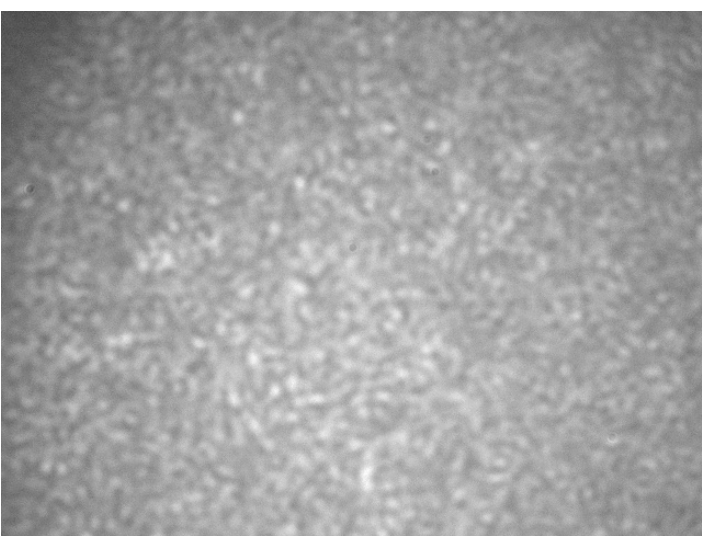

b)

Figure 4.1: Fiber optic speckle with a) Stationary binary diffuser b) Rotating binary diffuser at $60 \mathrm{~Hz}$

The stationary diffuser image has a speckle contrast of 27\%. Measuring the reduction of the contrast given various rotational speeds for this diffuser type was discussed previously in Section 3.3 and shows that rotational speed will not affect speckle contrast values. Rotational speeds of $60 \mathrm{~Hz}$ were used for all diffuser measurements in conjunction with the fiber optic element. Figure $4.1 \mathrm{~b}$ shows the image from a rotating binary diffuser. The measured speckle contrast is $18 \%$. The stationary grayscale diffuser is shown in Figure 4.2a with the rotating grayscale shown in Figure 4.2b. The stationary contrast measurement is $34 \%$ with the rotating contrast at $26 \%$. The contrast values show that the binary diffuser reduces the contrast greater than the grayscale diffuser which matches up with previous rotational diffuser results although there is a larger difference between the two contrast values than previously calculated [34]. 


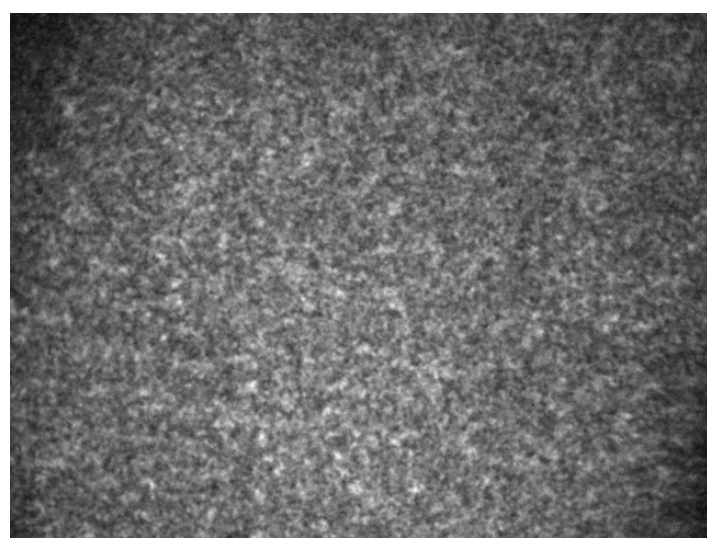

a)

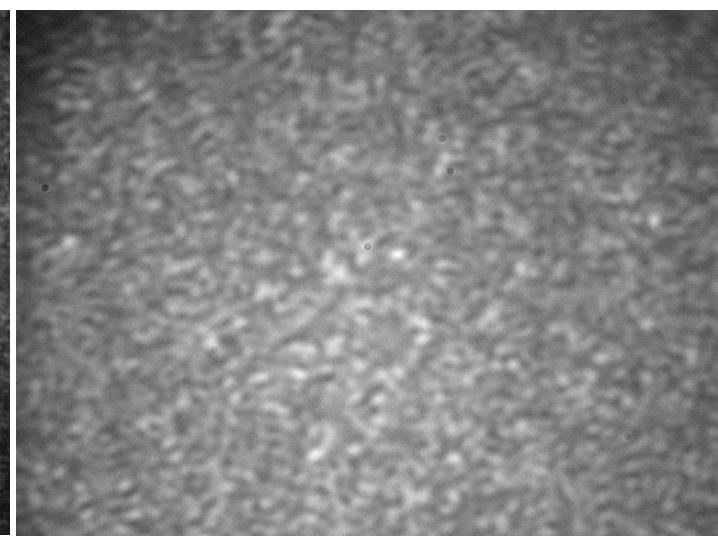

b)

Figure 4.2: Fiber optic speckle with a) Stationary grayscale diffuser and b) Rotating grayscale diffuser at $60 \mathrm{~Hz}$

As can be seen in the above images, larger secondary speckle is caused by the modes of the fiber. This can be viewed without the diffuser in place by just imaging the fiber directly using the same projection lens. Figure 4.3 illustrates this point below. It can be seen that the diffuser, when rotating, smoothes out these modes, although not completely. This is verified with the contrast results as the fiber only imaged produced a measured speckle contrast of $32 \%$. From the previously stated contrast results the grayscale diffuser, when stationary, increases the contrast instead of reducing it. This could just be an error factor given that the two values are so close together (within 5\% measurement error). Another viable explanation is that the speckle contrast value is actually increased because the diffuser's speckle compounds with the fiber's speckle in a disadvantageous way. Compared to standard speckle contrast for a polarized laser of approximately $70 \%$, the fiber itself reduces the contrast by a maximum of $50 \%[11]$. 


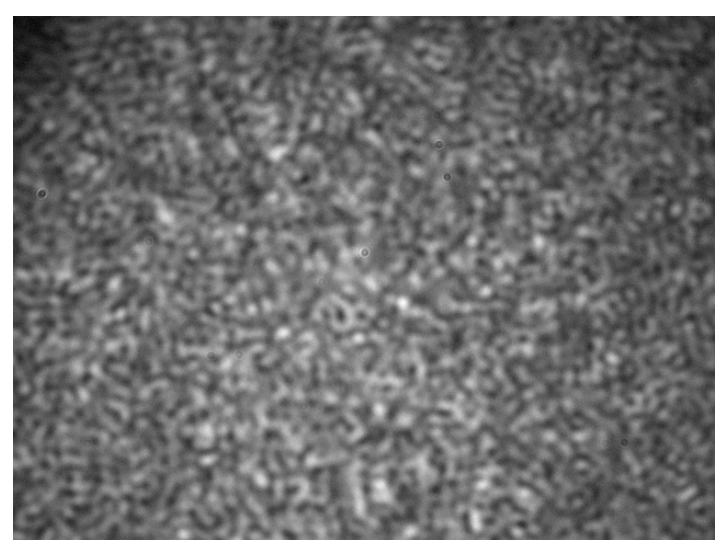

Figure 4.3: Fiber mode image without diffuser

Figure 4.4 illustrates the performance of all three wavelengths with both binary and grayscale diffusers, stationary, and rotating. The results are interesting to examine because unlike the blue diode, the green and red lasers did not need special optics to collimate the beam properly, meaning no fiber was used for either of these. This adds a layer of complexity because if the fiber speckle were eliminated or reduced in disparity the contrast of the blue would be on par with the other two result sets.

The ability to route a laser beam through a fiber instead of open propagation could be beneficial for small area direction and limiting the number of optical elements required. In addition, combining the three wavelengths into a single fiber would create a single optical system. The beam divergence from the fiber is wavelength dependent, which would cause matching errors with all three lasers in conjunction. Initial testing shows that extra aperture stops could alleviate this disruption. Initial investigations have been done with other diodes of differing wavelengths (specifically $635 \mathrm{~nm}$ and $532 \mathrm{~nm}$ - DPSS) using the same fiber optic lengths as discussed previously to determine contrast reduction results. Once the data has been analyzed, the next step will be the merging of the three wavelengths. Another aspect of the 
properties of lasers to acknowledge, in addition to the wave-front structure, is the polarization of the laser. The following section discusses polarization and its effect on speckle and the use to reduce speckle further when combined with diffractive diffusers.

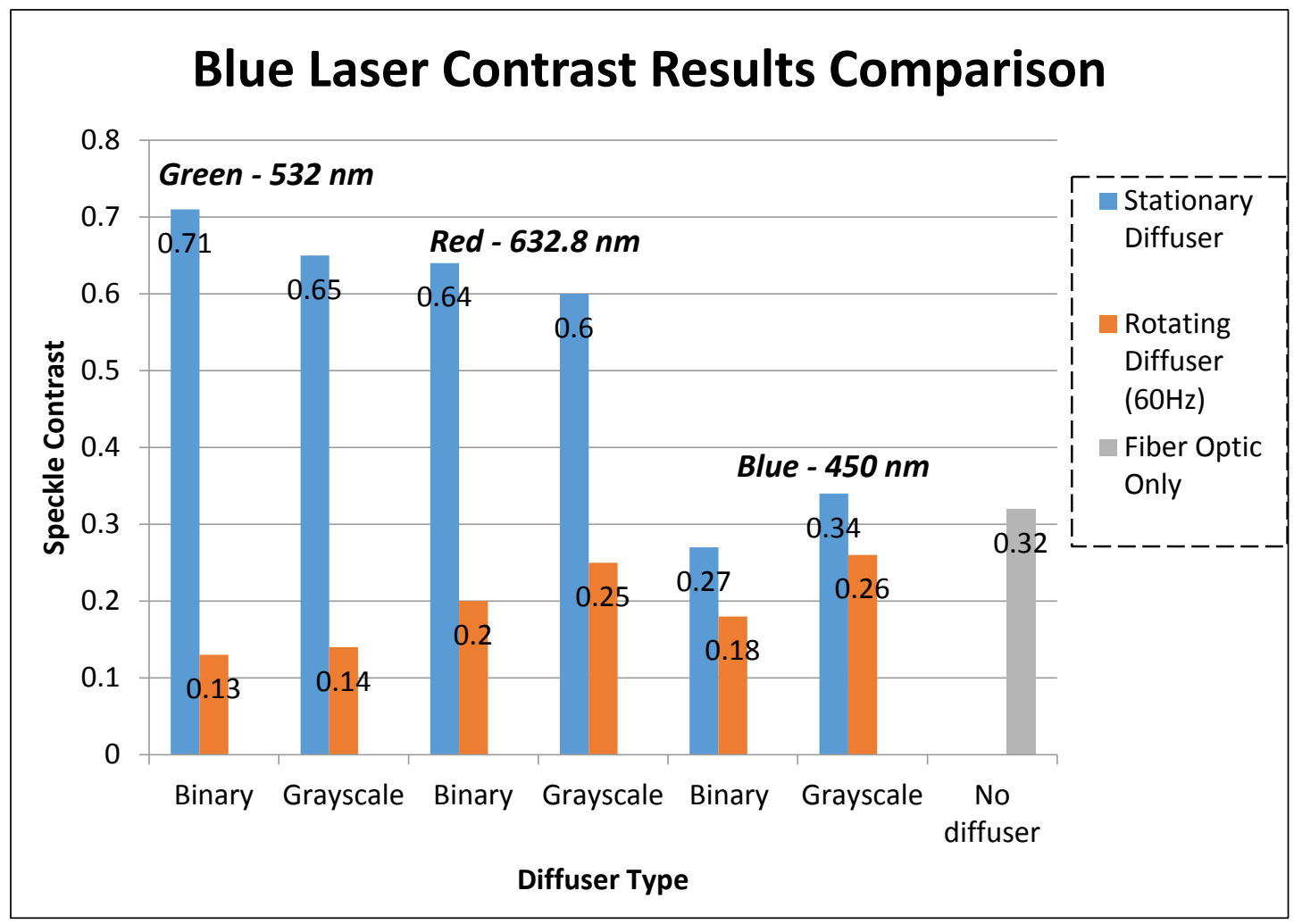

Figure 4.4: Graph of Comparison between the three laser wavelengths/sources with red and green laser values taken from Chapter 2

\subsection{Polarization Based Speckle Effects}

Polarization is a fundamental property of all electromagnetic waves. The relationship between the direction of the time-varying electric field and the direction of propagation determines the polarization state of any light. Non-coherent light sources, such as incandescent light bulbs, produce unpolarized light. However, coherent sources, often monochromatic, will have a specific polarization state and direction of 
polarization associated with it $[1,2]$. Lasers, in particular are generally classified as being polarized, referring to the fact that their electric field has a linear relationship with the direction of propagation. This specific state of polarization is referred to as linear. Linear polarization can exist in any direction orthogonal to the direction of propagation so long as it matches the plane of the electric field [2]. Circular polarization, while possible in coherent laser sources largely has to be implemented using polarization changing optical elements. While the polarization state of a laser is important information, the introduction of multiple polarization states has significant influence on the resultant speckle contrast.

A laser with "random" polarization has an electric field that contains multiple instances of linear polarization, in direct contrast to completely unpolarized light. Having random polarization is necessary, since multiple polarization states of coherent light can be separated from a single monochromatic laser source using polarizers and polarizing beam-splitters. Recombination of multiple linear polarization states results in a reduction in speckle determined by the angular difference in the direction of polarization. Specifically, two orthogonal linear polarization states will not interfere, even with coherent illumination. The noninterference between two light patterns from the same source causes a maximum speckle decrease of $\frac{1}{\sqrt{2}}$ [5] as discussed in Chapter 1 .

This technique for speckle reduction, while simple and beneficial requires additional components and more complex alignment in order to function. However, 
investigation is necessary as mixed with diffusers split-polarization could provide a substantial speckle reduction. The $532 \mathrm{~nm}$ DPSS laser with random polarization was used. Figure 4.5 contains the bench top setup needed to split the random polarization into two orthogonal directions.

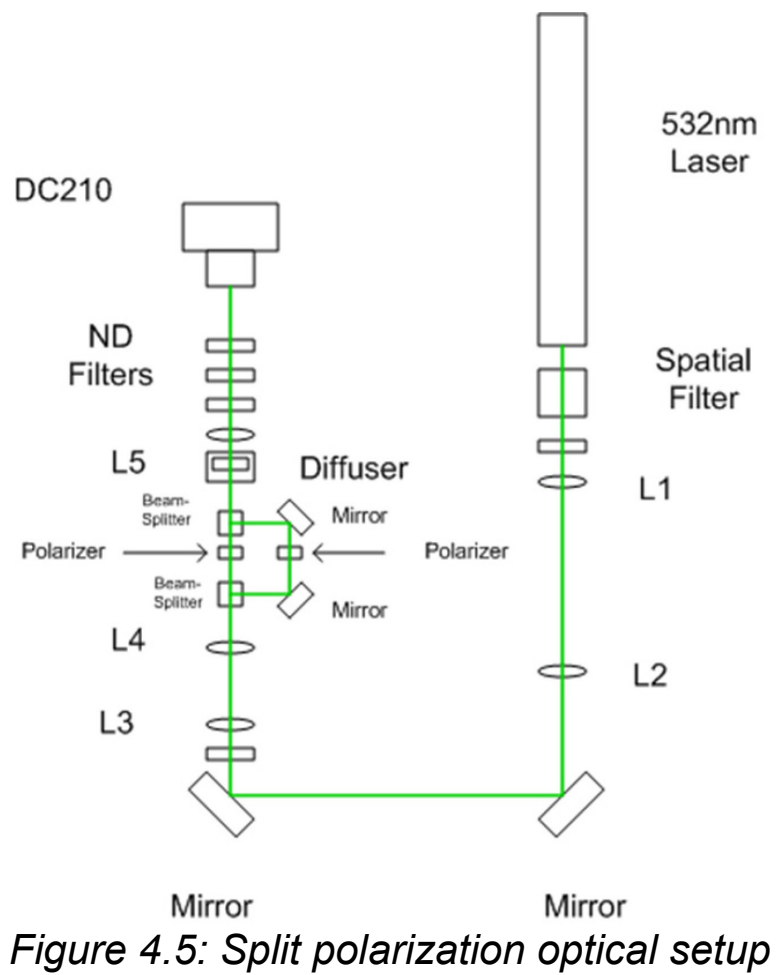

Figure $4.6 \mathrm{a} \& \mathrm{~b}$ show both grayscale and binary speckle images resulting from split polarization. Using this method speckle results of 0.40 and 0.16 were achieved with a stationary and rotating binary diffuser respectively. While consistent with previous speckle results for rotating diffusers, it provided a $45 \%$ speckle reduction for stationary diffuser. For the grayscale diffuser, speckle contrasts of 0.45 and 0.20 were obtained for both stationary and rotating diffusers. This is a $35 \%$ decrease in speckle contrast for stationary diffusers. This shows that while the reduction achieved from temporal averaging overwhelms any speckle decrease from splitting the polarization, 
the reduction achieved with stationary diffusers is of huge benefit. This matches well with the previously stated hypothesis.

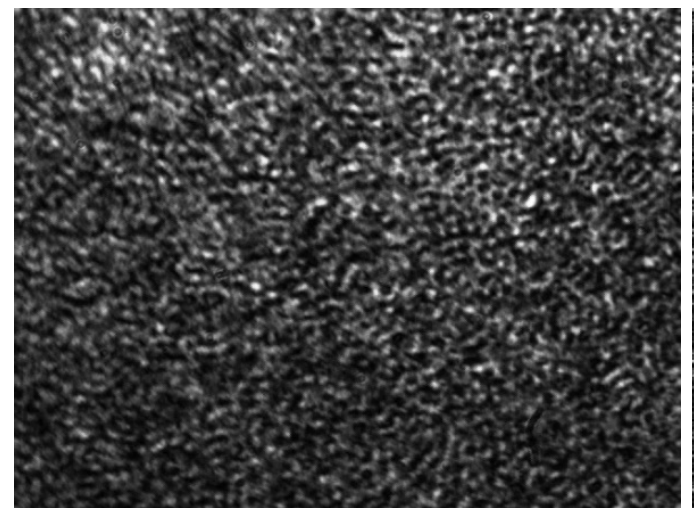

a)

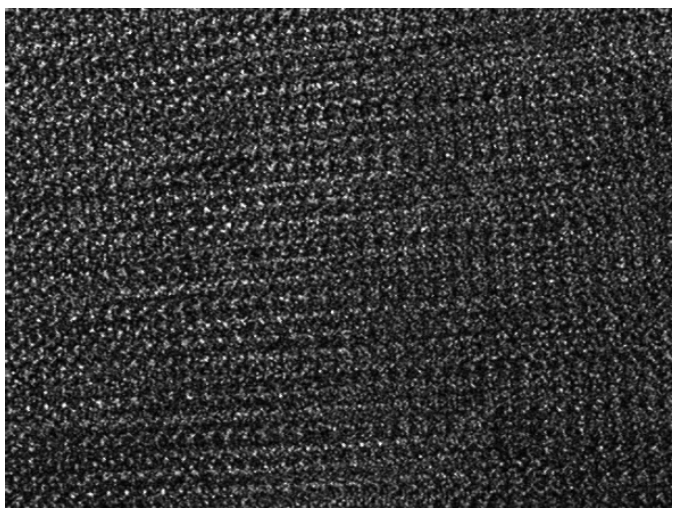

b)

Figure 4.6: Split polarization speckle image with a) a grayscale diffuser and b) a binary diffuser.

\subsection{Secondary Speckle Reduction Methods' Conclusion}

Both secondary speckle reduction methods were not as beneficial as would be preferred for our current rotating diffusers. However, both methods did decrease speckle $50 \%$ from a stationary diffuser. While rotating diffusers currently have some of the lowest speckle values, a moving part of any type would create vibrations in a handheld device that will disrupt the image. It would be best to move away from rotating diffusers in order to solve this design flaw. A rotating or vibrating diffuser would also create a possible mechanical failure point. Mechanical failure is especially problematic for small scale systems known as pico-projectors which already have a severely limited spatial allocation. Designing a diffuser that reduces speckle without the need for physical manipulation is preferred for any laser projection system. 


\section{Chapter 5: Simulating Diffusers using Fourier Optics}

\subsection{Simulation Requirements}

In order for temporal averaging to completely negate speckle for the human eye, sample rate of $23 \mathrm{~Hz}$, a diffuser must rotate a number approaching infinity all within 0.05 seconds. A rotating or vibrating diffuser would also be susceptible to mechanical failures, reducing product lifespan. Rotational or vibrational methods require additional supporting components, increasing implementation difficulty in picoprojectors which have severely limited spatial allocation. A diffuser that reduces speckle without the need for physical manipulation is ideal for laser pico-projection systems. Previous work on non-rotating diffusers have had limited success. A more recent article utilized a MEMS device for very small motion of the diffuser [13] where speckle reduction of 0.48 was achieved. Another method used piezoelectric benders to reduce speckle while limiting actual diffuser motion with a contrast of 0.16 [35]. The piezoelectric method, while non-rotational, requires two mechanically vibrating parts making the design susceptible to failure. Various contrast reduction methods $[11,12,16,34]$ have resulted in significant speckle contrast, but all require motion. Additionally, the majority of speckle reduction methods require large setups or devices that would need to be miniaturized for pico-projector application. There is a need to design a novel non-rotational, non-moving diffractive diffuser that is easily integrated into laser pico-projectors, while significantly reducing speckle contrast. To 
facilitate the design process, a simulation is needed to test and analyze a diffuser design prior to manufacturing which can be very costly. The focus of this simulation is mimicking the irradiance pattern resulting from a diffuser and any necessary projection optics. This chapter investigates diffractive optics modeling by first mimicking the irradiance output from the experimentally tested grayscale and binary diffusers, both rotating and stationary.

Modeling of propagating light in conjunction with diffraction techniques, a physics 'heavy' software package must be utilized. More generic (not geared towards optics) simulation programs were examined, including COMSOL Multiphysics ${ }^{\circledR}$ and RSoft ${ }^{\circ}$. Both are very popular for light propagation models, although each has its own specialty. RSoft ${ }^{\circledR}$ has a focus geared towards the smaller scale, specifically waveguide models (not exclusively however). The main benefit of using $\mathrm{RSoft}{ }^{\circledR}$ would be the rigorous electromagnetic modeling that could be attained. The problem is that it becomes practically useless when the scale is increased to the size of an optical diffuser and its diffractive output. An RSoft ${ }^{\circledR}$ simulation of this magnitude would not be possible due to computation restraints. The method used to model optical propagation in this regime would also fail because of the lack of boundaries provided by the diffuser simulation. The final contender was COMSOL ${ }^{\circledR}$, a more general electromagnetic/physics modeling program. COMSOL $®$ is more of a CAD program used to create a model and determine how general electromagnetic waves interact with the physical model. Although this would be an interesting technique for analyzing the phase diffuser, it would not be relevant to the current project due to the 
lack of ability to create coherent visible light. A few other modeling software systems follow the same type of method as COMSOL ${ }^{\circledR}$, meaning none of these could be considered either.

Zemax ${ }^{\circledR}$ is currently on the forefront of optical modeling software. A physical model of the diffuser could be created and then using the non-sequential ray tracing mode, a Gaussian "laser" beam would illuminate the diffuser and resultant irradiance propagated onto a detector. The biggest drawback to using this software package is the complexity involved in making alterations to diffuser designs. Zemax ${ }^{\circledR}$ operates by allowing the user to create an optical surface with any desired physical property. A multi-level phase diffuser would require the creation of multiple surfaces per diffuser instead of just the two needed for lenses. A whole new diffuser would need to be created for each slight change, including phase level changes or physical size adjustments. An open ended mathematical approach is preferred so that diffuser layouts can be changed rapidly to account for design alterations.

MATLAB ${ }^{\circledR}$ is a computation program (as opposed to model creation) that runs similar to $\mathrm{C}$ programming. This allows MATLAB ${ }^{\circledR}$ to remain very open ended for simulation design (if a system can be described by a series of mathematical equations than that system can be replicated through simulation using MATLAB $\mathbb{R})$. This satisfies the requirements for a large scale physical simulation. In addition, light propagation and diffraction can be modeled through equations (although advanced systems could be very difficult to create). Optical propagation can be accomplished through the use of Fourier optics, an optics modeling theory based on the use of 
Fourier transforms to determine generated field distributions as demonstrated in Section 5.3 of this chapter. The most important consideration for using Fourier optics with MATLAB ${ }^{\circledR}$ is the necessity of sampling. Due to the constraints of a non-real system, the simulation must be constructed in discrete form. The laser light must be sampled properly in order to both create the proper system (matching a physical system - too few samples) and remain stable within the software environment (stop computation overflow - too many samples) [36].

A few distinct difficulties may arise within the work being completed through MATLAB ${ }^{\circledR}$, especially computationally based ones. Ideally, a fast enough computer would alleviate any computation or memory concerns, but lacking that long running times may persist for the necessary MATLAB $®$ functions to manufacture results. Secondly, any and all array formats will remain 2-dimensional for simplicity sake. At this point in time, considering a 3-dimensional optical simulation (i.e. takes the depth of the screen into consideration) seems to be counterproductive from the original idea of merely creating a working "prototype" reproduction of the physical system.

The discrete Fourier transform (DFT) in MATLAB ${ }^{\circledR}$ can be calculated using the fast Fourier transform (FFT) or 2-D FFT for arrays. Essentially, the Fraunhofer propagation mechanism - and to some extent the Fresnel propagator - can be defined as simply the Fourier transform of the transmittance function, once again defined by the grating. Therefore, taking the FFT of our aperture, with some scaling factor applied, the desired output fields will be created. A scaling on the axis of the final 
image will be required based on the distance, $z$ and the sample spacing $d x \& d y$, respectively [36].

\subsection{Fourier Optical Model for Diffusers}

This diffuser is modeled as a single scattering phase screen, written in the form of a transmission aperture, given by Equation 5.1 [37]. Figure 5.1a illustrates the placement of the diffuser in line with the initial beam and followed by the imaging screen in the $(x, y)$ plane, which has the varying phase pattern across the diffuser surface.

$$
t_{A}(x, y)=e^{\varphi(x, y)}
$$

The simulation will revolve around mimicking the operation of two distinct diffuser types: binary and grayscale. The binary diffuser contains only two distinct phase level sections, which generates sharp edges in the output image. The grayscale diffuser takes advantage of 64 discrete phase levels creating a smoother granular profile [34]. Both types are designed to reduce the visual degradation caused by speckle through rotation. They are both modeled with an array of 2000x2000 pixels. Each diffuser is composed of individual cells replicated across the surface as demonstrated in Figure 5.1b. The binary diffuser's unit cell is an order 16 matrix, while each Grayscale unit cell is a random organization of the 64 phase levels. These two unit cells are approximations of the physical diffusers that were used for experimental measurements. Further investigation of the diffuser model will be discussed in the next section. 
The output from the aperture can be found by using a Fourier approximation for distance propagation, such as the Fresnel equation [38]. Treating the mathematical computation as a linear system allows the separation of code to flow freely with the functional partitions. The system can be broken up into multiple parts as well based on the needs of the simulation. In this instance it is helpful to separate the Fourier optics propagation and the diffuser rotational program from the individual diffuser models allowing the diffuser models to be interchanged without rewriting large amounts of the simulation.

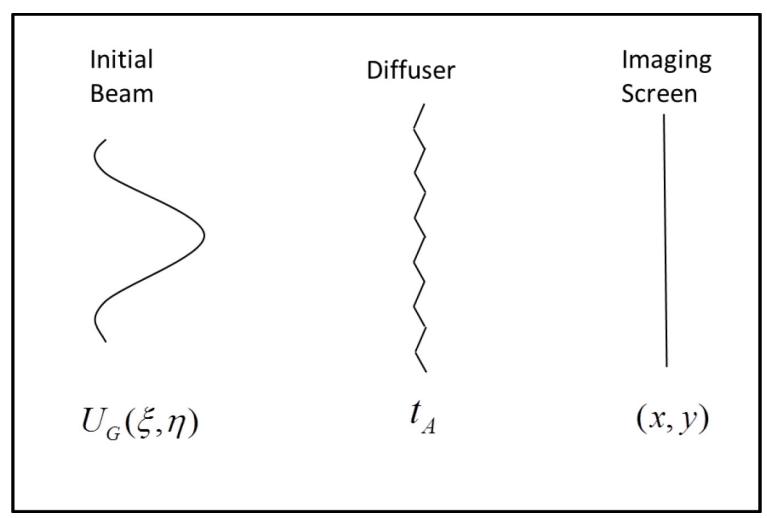

a)

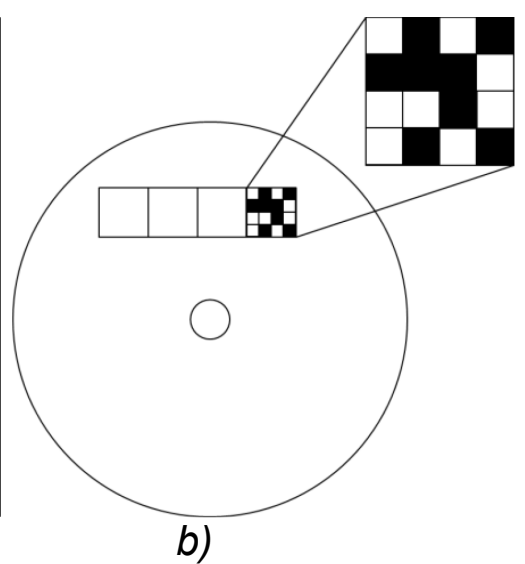

b)

Figure 5.1:(a) Diffuser model layout; (b) Diffuser unit cell layout

The initial beam is considered to be a collimated monochromatic Gaussian beam ${ }^{1}$, as demonstrated in Figure 5.2, which is defined as [39]

$$
U_{G}(\xi, \eta)=e^{-\frac{[(\xi+a) \Delta \xi]^{2}+[(\eta+b) \Delta \eta]^{2}}{w_{0}^{2}}}
$$

where the position $(a, b)$ is the center of the Gaussian beam, is the beam waist, and $(\Delta \xi, \Delta \eta)$ represents the physical size divided by the number of pixels in each

\footnotetext{
${ }^{1}$ Gaussian beam code provided by Kyle Drexler, former ECE graduate student
} 
direction. The laser wave front will be the size reference from which the rest of the simulation is measured. The xy-plane is the plane of incidence of the wave-front. The Gaussian beam is propagated through the diffuser in two steps. First, the wave is multiplied by the transmission aperture, $t_{A}$ adding a phase displacement to the initial beam. The output of this calculation, shown in Equation 5.3, represents the field directly after the diffuser [40].

$$
U_{1}(\xi, \eta)=U_{G}(\xi, \eta) \times t_{A}(\xi, \eta)
$$

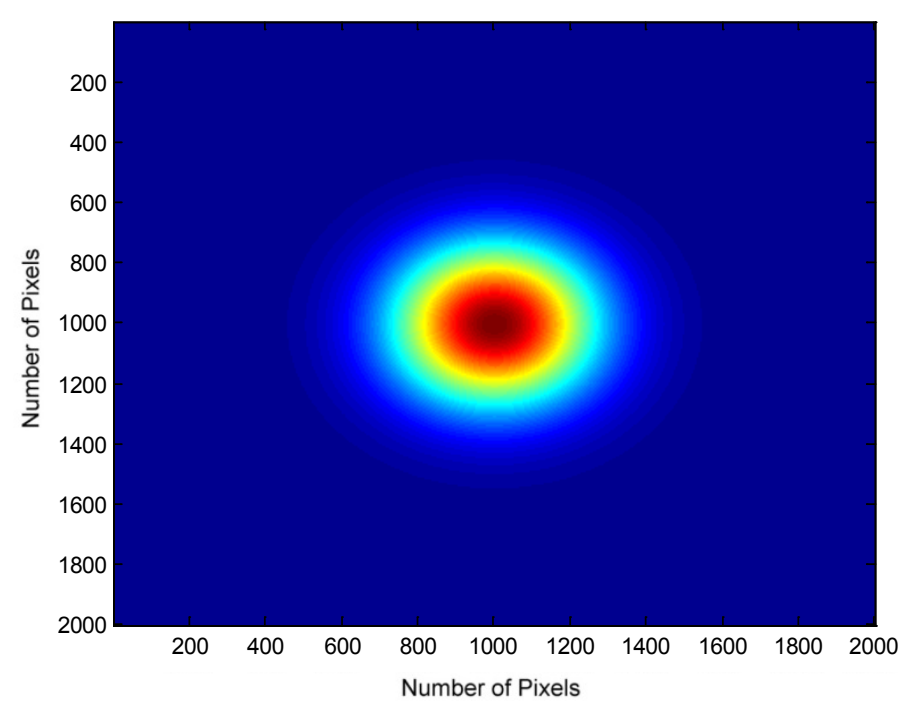

Figure 5.2: Gaussian Beam with linear width of $1.5 \mathrm{~mm}$

The second part of the simulation involves the Fourier propagation of the field right after the diffuser onto the $(x, y)$ image plane. This is accomplished through the Fresnel approximation of the Rayleigh-Sommerfield equation [37, 41]. A near-field propagation method is needed since the output of the diffuser would be imaged onto a projection device such as a MEMS scanner or MLA, within close proximity. The Fresnel approximation is given in Equation 5.4 where $\lambda$ is the wavelength, $k$ is the 
wavenumber, $z$ is the propagation distance along the optical axis, and $(\xi, \eta)$ is the aperture plane. The wavelength is chosen to be $535 \mathrm{~nm}$, representing a generic green laser diode at the center wavelength of the visible spectrum.

$$
U_{2}(x, y)=\frac{e^{j k z}}{j \lambda z} \iint U_{1}(\xi, \eta) e^{j \frac{k}{2 z}\left[(x-\xi)^{2}+(y-\eta)^{2}\right]} d \xi d \eta
$$

There are technically two distinct ways to define what is known as the Fresnel propagator. The first is termed the "Transfer Function" (TF) and the second as "Impulse Response" (IR). The TF propagator is described by the following [36]:

$$
H\left(f_{x}, f_{y}\right)=e^{j k z} e^{-j \pi \lambda z\left(f_{x}^{2}+f_{y}^{2}\right)}
$$

Meanwhile the impulse response is [36]:

$$
h(x, y)=\frac{e^{j k z}}{j \lambda z} \cdot e^{\frac{j k}{2 z}\left(x^{2}+y^{2}\right)}
$$

The main difference between these two propagator functions is the sampling requirements. The TF version requires a large real space sampling value to work properly and the linear resolution needs to remain large per Equation 5.7. In opposition, the IR sampling size requirement is the inverse of Equation 5.7 and must remain sufficiently small in real space while having a large frequency resolution [21, 36].

$$
\Delta x>\frac{\lambda z}{L}
$$


For the discrete version, $\Delta x$ is the linear phase element size, $\lambda$ is the wavelength, $z$ is the propagation distance in pixels, and $L$ represents the total physical side length of the array. The final field image cannot be generated too close to the diffuser without causing quadratic errors in the exponential component. Using previous values for the diffuser aperture size and imaging array size, 1600 and 2000 pixels respectively, a proximity limit of 4500 pixels is calculated using Equation 5.8. This is considered the near-field region of measurement [42]. While the Fresnel integral does work in the far-field, the loss of image clarity due to beam expansion makes it less convenient to use and the model refrains from approaching the Fraunhofer region, defined by Equation 5.9 [42].

$$
\begin{gathered}
z^{3}>\frac{\pi}{4 \lambda}\left[(x-\xi)^{2}+(y-\eta)^{2}\right]_{\max }^{2} \\
z>\frac{k\left(\xi^{2}+\eta^{2}\right)_{\max }^{2}}{2}
\end{gathered}
$$

The final section of the simulation involves the rotation of the diffuser and the modeling of a camera or capture device. Using previous data, the diffuser can be sufficiently operated at $60(+/-0.5)$ revolutions per second [34]. In addition, the camera will operate at $30 \mathrm{~Hz}$, to mimic the eye's refresh rate or sampling time of 23 frames per second [10]. To mimic the diffuser rotation, the diffuser matrix array will be rotated prior to propagation of the field $U_{1}(\xi, \eta)$. The final field is then normalized by the total number of images, $h$. The intensity image is then found by squaring the 
absolute value of the field, $U_{F}(x, y)$. A capture device integrates on the order of $10^{20}$ photons for a single image during a predetermined exposure time.

In order to represent the large number of photons incident upon a detector, an approximation is resolved through a series of Monte Carlo simulations. A generic noise pattern was created and then rotated a single revolution. The images were rotated and integrated at various degree increments ranging from 0.01 degrees to 60 degrees. The resolutions of the various images were compared against one another until the individual noise parameters were indistinguishable which identified the degree resolution required for the simulation. The degree resolution was defined at 0.75 , requiring 480 total images integrated together to create a single frame.

Comparing the speed of the diffuser at $60 \mathrm{~Hz}$ to the camera integration speed of 30 $\mathrm{Hz}$ it is identified that 2 rotations are completed for every single image frame. This will require at least 144000 distinct images to represent a 10 second video capture. Now that the conditions for the models have been established, output fields for the diffusers can be found.

\subsection{Modeling the Diffusers}

Equation 5.7 demonstrates the linear size limitation given this array size and a $z$ depth of $2 \times 10^{4}$ pixels. Every individual phase element will consist of 25 pixels for each diffuser model. The minimum phase element size is 2.8 pixels for accurate sampling. This has been increased to be arranged evenly and symmetrically across the diffuser surface $[36,43]$. A distance mapping along the optical axis can be characterized along the z-axis. Every square pixel is related to a physical length by $2.5 \mu \mathrm{m}$. This mapping 
can be established for the propagation axis, $\mathrm{z}$. This framing scale is maintained for all arrays unless distinctly noted on the axis of the image. The phase screen arrays are demonstrated in Figures 5.3a \& 5.3b.

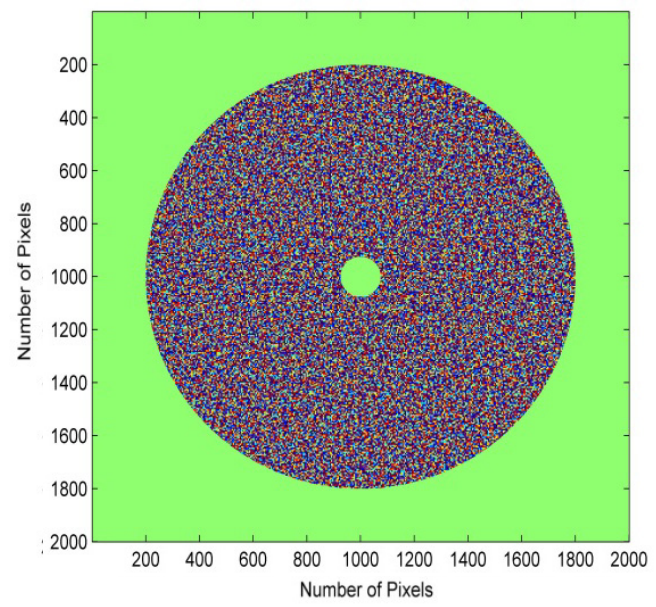

a)

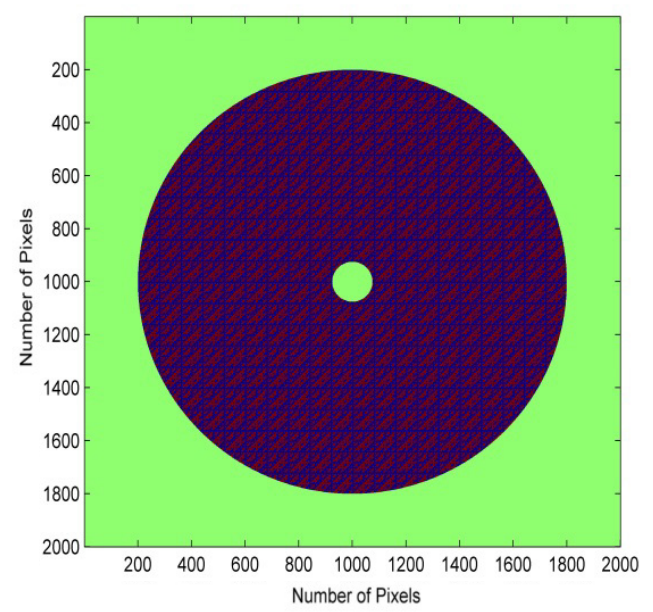

b)

Figure 5.3: (a) Grayscale diffuser array; (b) Binary diffuser array

The binary diffuser needs two distinct phase levels to create the distinct edge shape. These levels do not necessarily need to be restricted to certain values, but the power from the diffuser needs to fall primarily into the first order of the image. Various levels between $\pi$ and $-\pi$ for the binary transmittance function were used prior to the 
full simulation to determine power distribution. Using a simple version of the Fresnel propagator, the output image can be evaluated at some distance away from the diffuser. The 3 images shown in Figure 5.4 exemplify the differences, as well as similarities, of the output when changing phase levels.

The first image (Figure 5.4a) shows a standard binary grating with $\varphi(x)=\pi / 2$ or $-\pi$ /2. The word standard merely refers to the idea of $j$ and $-j$ as being the quintessential imaginary phase values. Next shown is $\varphi(x)=\pi / 4$ and $-\pi / 4$. This image (Figure 5.4b) shows a more distinct brightness because the pattern has been slightly adjusted. There is a distinct difference between the two images caused by the decrease in phase difference of the two levels. The phase difference actually illustrates a bit less spread of a diffraction pattern, more than likely because of the increase in coherency (or rather the smaller difference of phase). This change in diffractive angle is illustrated to a greater degree with the third image (Figure 5.4c). This version of the binary diffuser has $\varphi(x)=\pi / 8$ or $-\pi / 8$. It is worth noting that the intensity profile of the image has not shifted in space due to the phase changes. This is an important aspect of the binary diffuser, though not a unique one. 


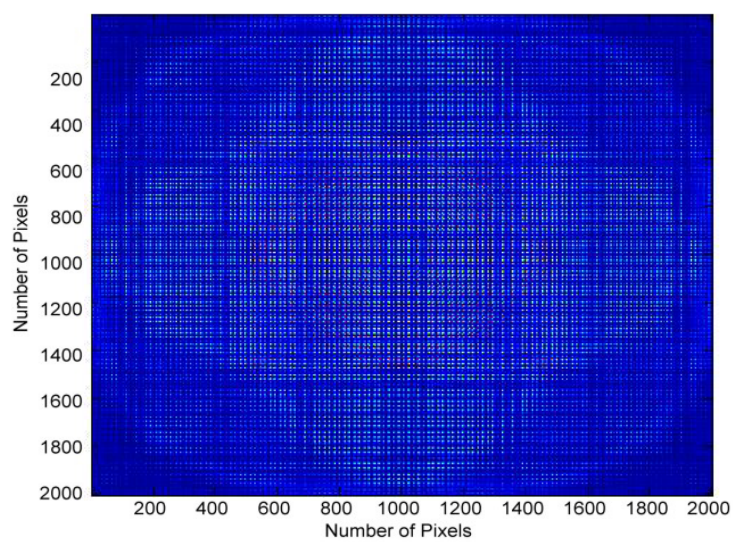

a)

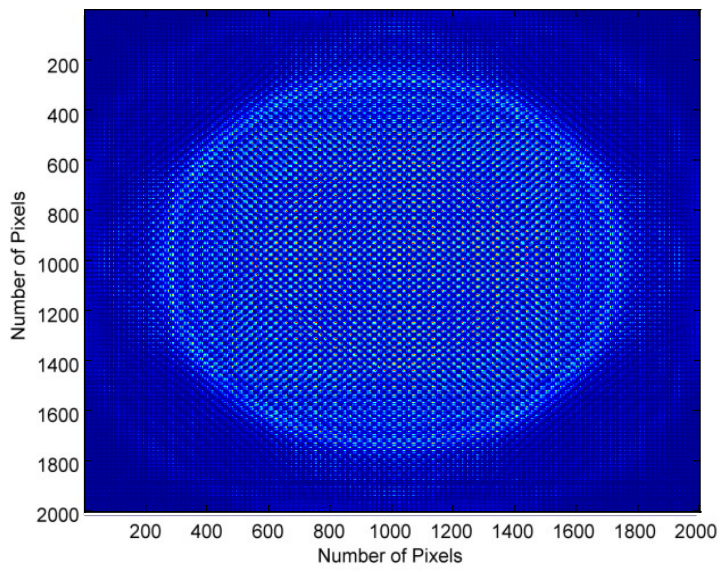

b)

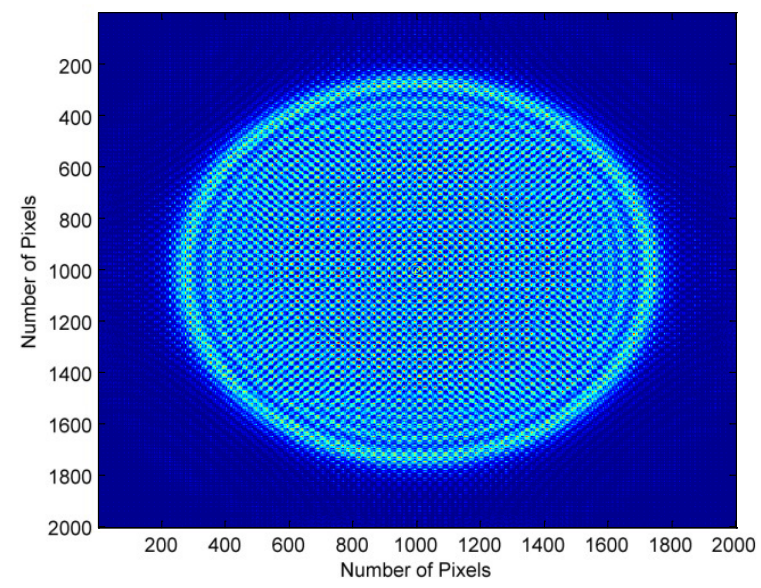

c)

Figure 5.4: Simulated output of binary diffuser with phase levels of a) $\pi / 2 \&$ $\pi / 2$ b) $\pi / 4 \&-\pi / 4$ c) $\pi / 8 \&-\pi / 8$ 
The grayscale diffuser model has an element of randomness which causes the more obvious speckled image pattern. The values of the phase (64 levels) do not change this speckle pattern. For this situation it is assumed that all 64 phase elements are evenly distributed unique values between $\pi$ and 0 which keep the power situated optical center. It could be feasible to slightly shift the power off-center using phase levels in a smaller region of the space, (i.e between 0 and pi/64.) However, image scaling will occur similar to the binary diffuser affect previously discussed. Figure 5.5 illustrates the intensity pattern that occurs at a distance of roughly $12 \mathrm{~cm}$. (which matches physical distances from experimentation) from the diffuser surface using a standard Fresnel propagator with sampling of 1 pixel being representative of $6.25 \mu \mathrm{m}$ $(+/-1 \mu \mathrm{m})$. An important concept to note is that only the lowest order or central spot is of interest in this situation and thus the outer "shell" of the intensity pattern is just noise that can be essentially ignored.

A similar issue does occur for the propagated grayscale pattern. Normalized, the image intensity distribution is roughly of the same value, which means scaling is needed for proper analysis. However, it is hypothesized that this scaling issue (which now occurs for both types of diffusers) may be caused by the propagator model used. Based on previous instances, it has been assumed that the Fresnel propagator could roughly provide an approximation for the diffraction model but this may not be the case. As previously stated, certain limitations on $\Delta x$ cause one version of the propagator to not function properly (no propagation occurs). A similar limit could cause the propagator to fail for a certain number of phase elements (at one time). 


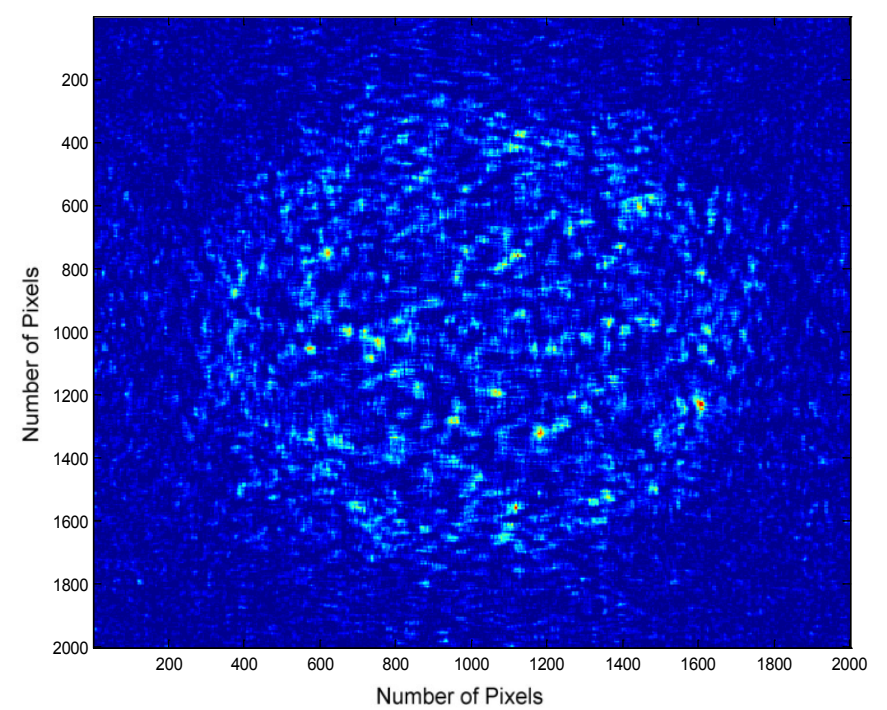

Figure 5.5: Generic Grayscale diffuser model at $10 \mathrm{~cm}$

The sampling restriction is of concern due to the size of the phase elements in the model. The phase elements pixel size (number of pixels representing each element) needs to be increased in order to avoid aliasing in the final output image based on Equations 5.7 and 5.8. Essentially, by increasing element size the number of sampled values corresponding to that element increases and a large overall sampling size is incurred. Two versions of propagated images, for the binary diffuser, with improper sampling are illustrated in Figures 5.6a \& 5.6b.

Figure 5.6a is the off scaled version, which means the propagation code does not properly scale the sampling values and causes errors in the actual propagation. It does look similar in pattern to the over-sampled/correctly sampled version, but the difference lies in the lack of stability for the under-sampled propagator model. Although certain images will possess qualities that resemble the proper model, they will not be able to output this image pattern consistently. The second image, Figure 
$5.6 \mathrm{~b}$ is one with proper propagation factors but the elements of the phase diffuser are too small (in both relation to the diffuser and relation to overall sampling) and thus cause scalar diffraction theory to fail. Aliasing occurs along the edges of the image caused by the FFT code not being defined with enough information (under-sampling).

This same idea of maintaining large enough element size or small sample size needs to be adhered to for the grayscale as well. Although similar to the results of the binary, the biggest difference is the randomness caused by having multiple phase levels. This causes a more obvious generic speckle pattern, which is still not consistent due to the stability issue of the propagation from under-sampling. Figure 5.7 is an under-sampled image of the propagated grayscale diffuser where the correct version can be viewed in Figure 5.5.

The concept of rotating the diffuser given a certain rotational speed, in cycles/second or hertz, will be maintained and copied only to provide context to the non-physical model. Using MATLAB's built in imrotate command, one can rotate the image while maintaining specific array size for any given degree increment. In order to mimic a camera capturing this rotation for a certain time scale (a camera will take an image at an integration time of $1 /$ frame rate), a certain number of images will need to be added and normalized to create the rotated diffuser image. 


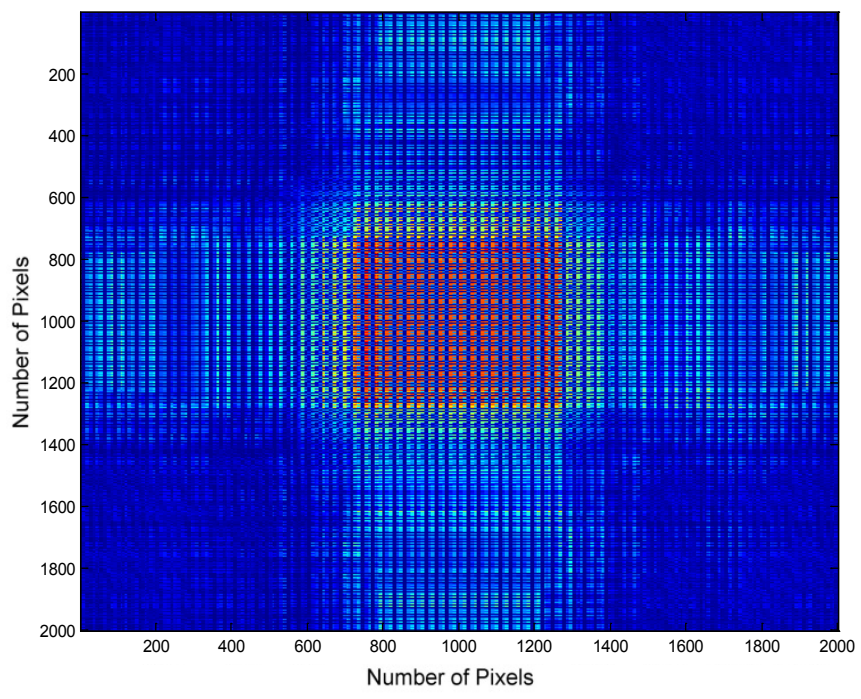

a)

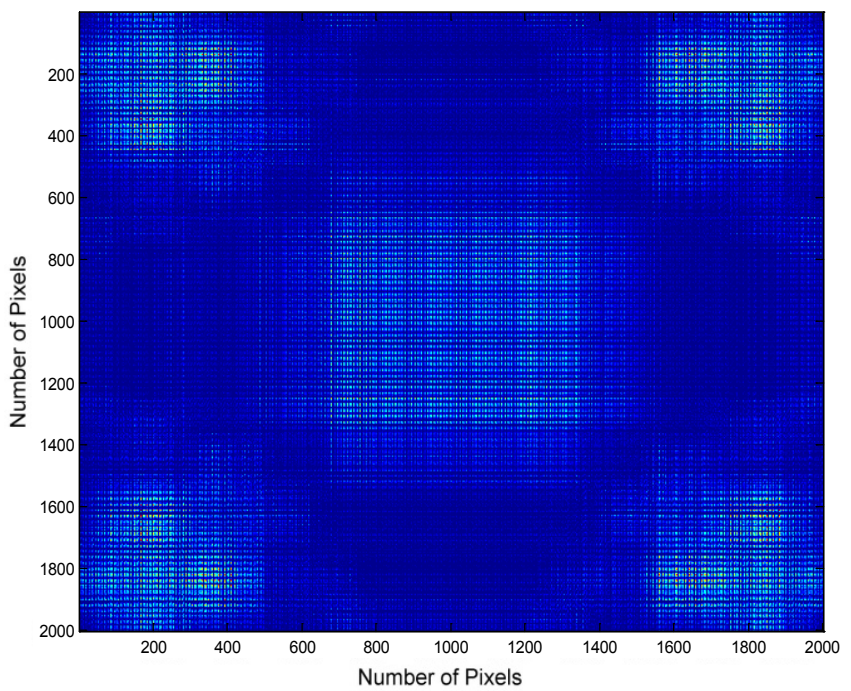

b)

Figure 5.6: a) Under-sampling caused by element size; b) Incorrect scaling of Fresnel propagator 


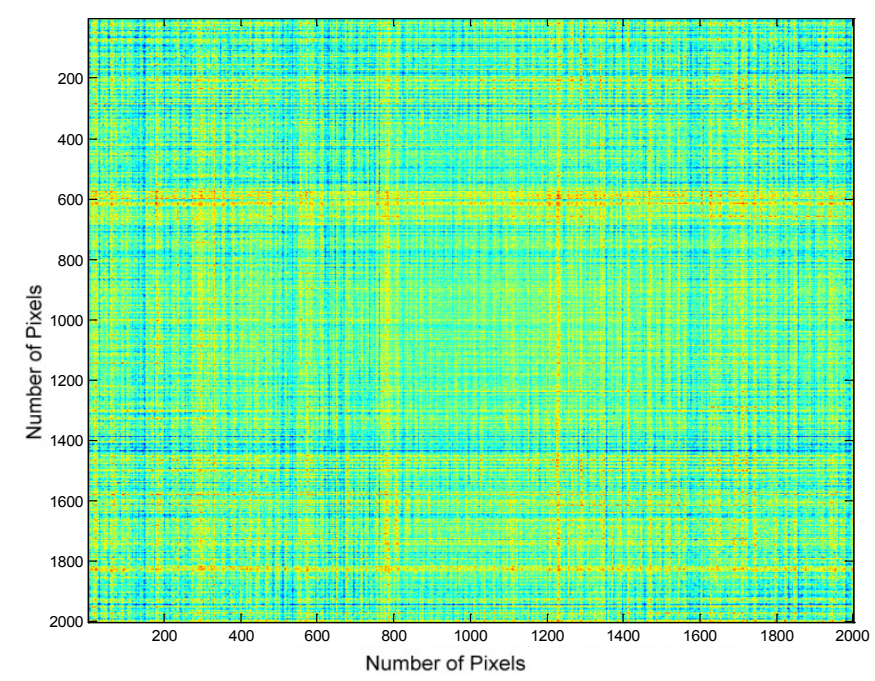

Figure 5.7: In-proper scaling/sampling of Grayscale Diffuser

A simulation was run where varying degree increments were utilized to determine an appropriate value for use in the simulation. A full rotation (360 degrees) of a generalized speckle pattern was imaged for decreasing degree increments. As the degree increments shrank, the speckle image became more and more smoothed, eventually reaching a type of exponential plateau. The beginning of this plateau is the best value for the degree shifts that can be used, as it will provide an approximation to real world light field integration while keeping computational constraints to a minimum. The following 2 images, Figures 5.8a \& 5.8b depict first a degree size too large (not fully integrated) and then an image with degree size that will be used for the experiment, $\Delta \theta=0.75$ degrees.

Initially, a beam wave-front and diffuser were created and were incident against the diffuser as the diffuser was rotated. The field from the output of the diffuser was propagated a given distance onto the output image plane. 


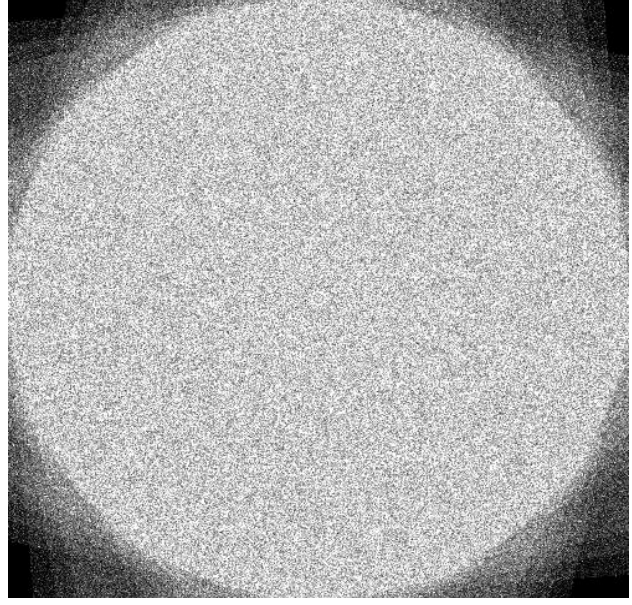

a)

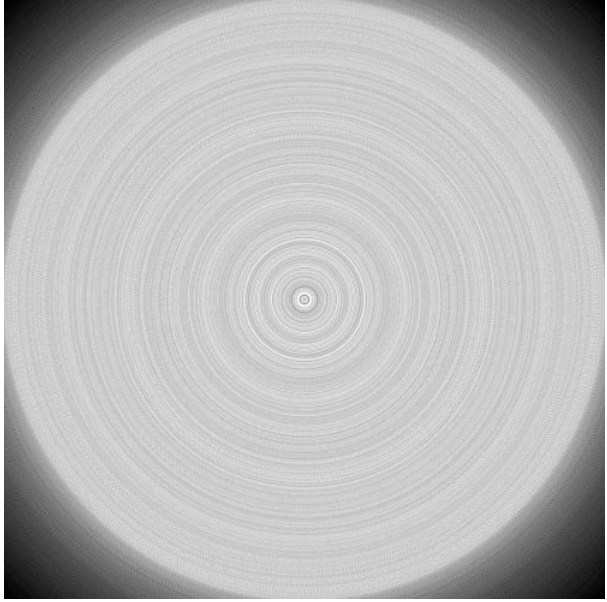

b)

Figure 5.8: Rotating speckle image with a) $\Delta \theta=50$ b) $\Delta \theta=0.75$

\subsection{Speckle Contrast Results}

Speckle Contrast calculations were made for the stationary and moving diffuser images using Equation 3.2. The images for the stationary diffusers are shown below in Figures $5.9 \mathrm{a} \& 5.9 \mathrm{~b}$ for the binary and grayscale versions, respectively. The central spot of the diffuser is the zero-order diffractive mode. Most diffractive optical elements will consist of some form of zero-order mode and are considered to be the DC portions of the element. An optimum diffuser will minimize this zero-order and smooth out the overall output profile of the speckle. The first order of the element contains the majority of the incident power, $90 \%(+/-5 \%)$ as measured experimentally, as well as the highest speckle reductive properties. The speckle contrast is calculated for this first order spot of the diffuser image. The binary version has a contrast of $0.76(+/-0.07)$ and the grayscale version has a contrast value of 0.74 $(+/-0.07)$. These values correspond to data taken from intensity images of the simulation. 


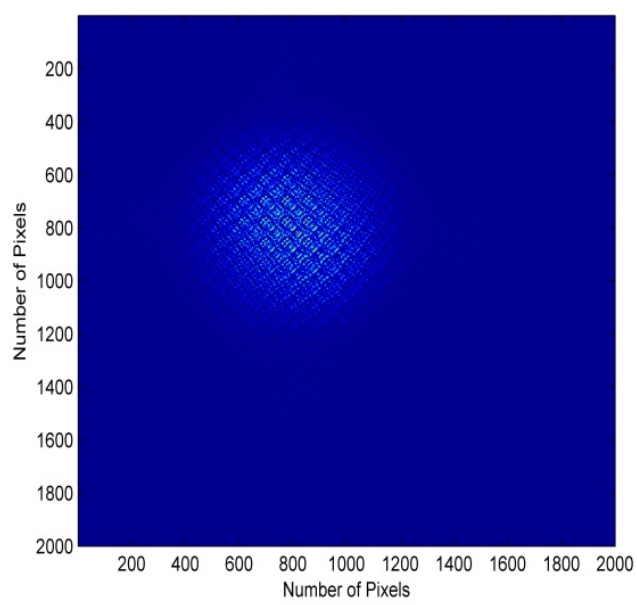

a)

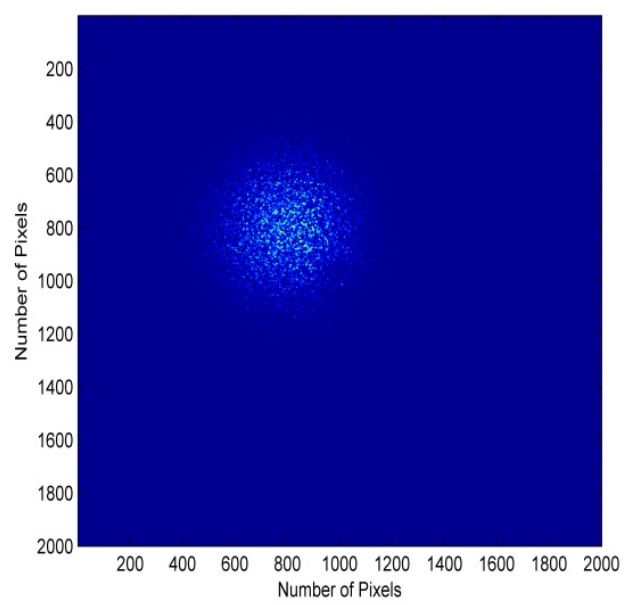

b)

Figure 5.9: (a) Stationary binary diffuser; (b) Stationary grayscale diffuser

Close up versions of the central order of the diffuser image are shown in Figures $5.10 \mathrm{a} \& 5.10 \mathrm{~b}$. This is the physical representation of a focused image from the diffuser. They are 300x300 pixel array snapshots of the total diffuser image from above. This can be directly compared with bench top experiments taken using binary and grayscale diffusers with a $532 \mathrm{~nm}$ DPSS laser [34]. The camera used for the physical experiments had a 480x640 pixel array. The stationary, focused diffuser images are shown in Figures 5.10c \& 5.10d. The contrast values for the bench top experiment are $0.77(+/-0.07)$ for the binary diffuser and $0.68(+/-0.07)$ for the grayscale version. The contrast values themselves are accurate to within $10 \%$. The images themselves, however, are not as comparable. The simulated versions have a more rigid structure while the bench top images are more fluid and organic, specifically related to the binary diffuser image. It is believed that this is a result of the inputs for the creation of the individual diffusers. The contrast results alone provide enough accuracy for the simulation to be acceptable. 


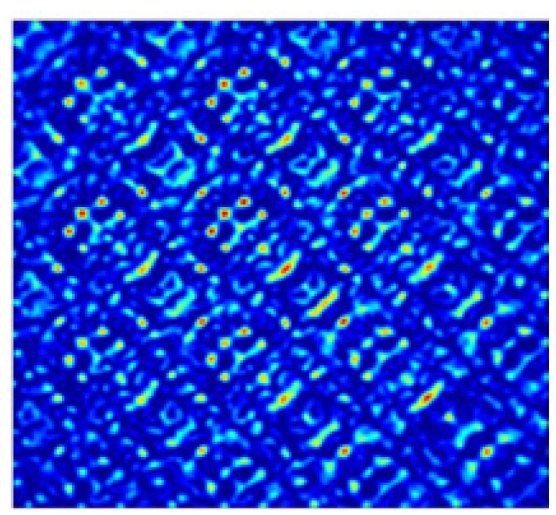

a)

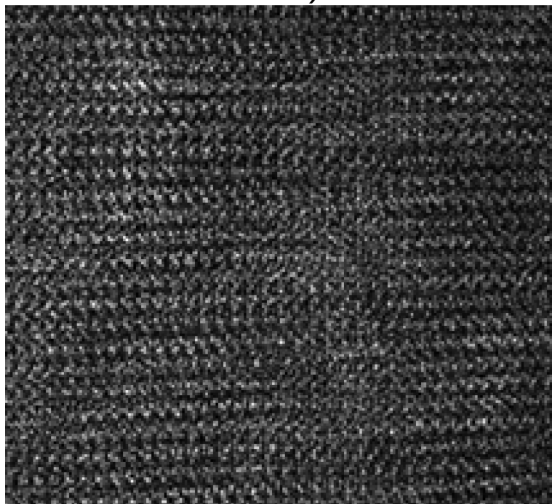

c)

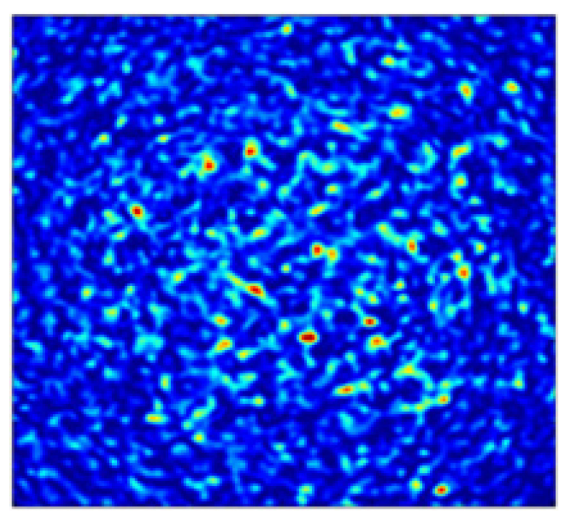

b)

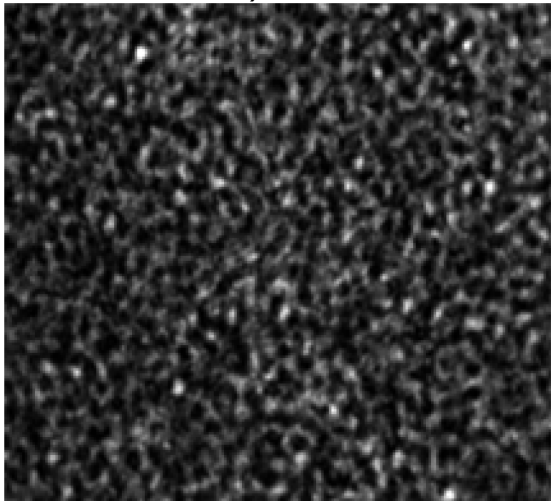

d)

Figure 5.10: (a) Focused, stationary binary diffuser; (b) Focused, stationary grayscale diffuser; (c) Physical binary diffuser output intensity image; (d) Physical grayscale diffuser output intensity image

The simulation was completed by creating rotating variations of the diffuser patterns and propagating them onto the image plane. The parameters were set to mimic the rotational speed of the diffuser as $60 \mathrm{~Hz}$. The speckle was only measured for the central order as it was for the rotating diffuser images. The simulated images are shown in Figures 5.11a \& 5.11b. The calculated contrast values for the binary and grayscale images are $0.297(+/-0.04)$ and $0.276(+/-0.04)$, respectively. The contrast values found in the bench top experiments were slightly less at $0.18(+/-0.06)$ for the binary and $0.14(+/-0.06)$ for the grayscale versions. This relates to a contrast 
difference of around $10-13 \%$ contrast reduction between simulation and actual results.

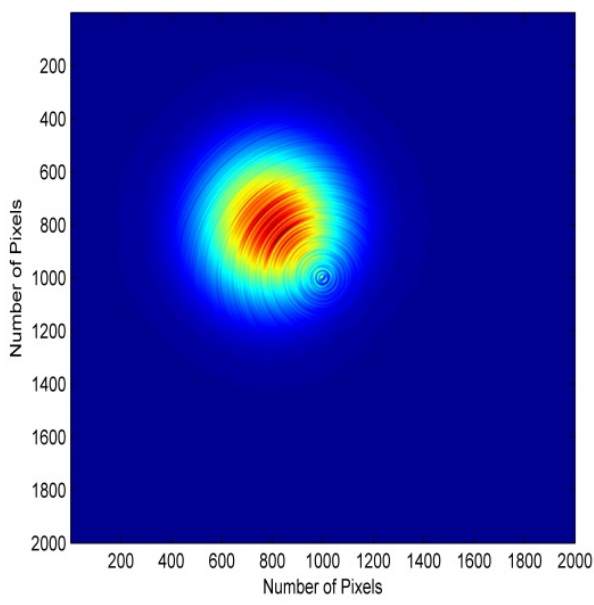

a)

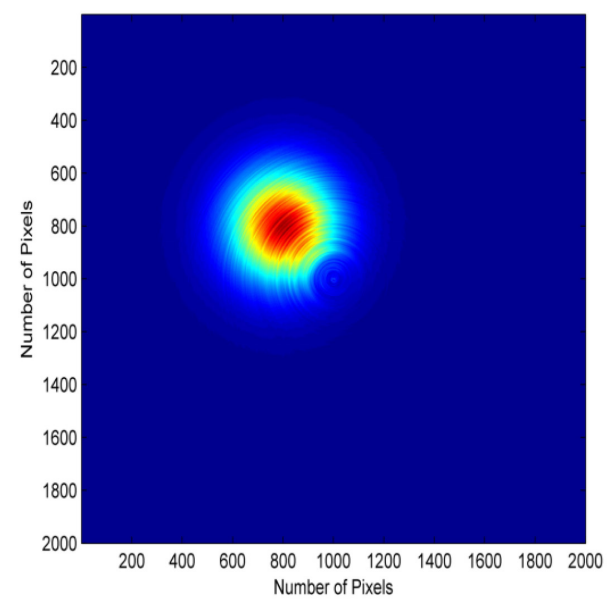

(b)

Figure 5.11: (a) Rotating binary diffuser; (b) Rotating grayscale diffuser

It is assumed that a single polarization is incident on the diffusers and that the diffusers are polarization maintaining. Previous research showed that the polarization incident on the diffuser did not have an impact on the final contrast results [34]. This is an approximation that can be changed in the simulation to ensure the theoretical and experimental results match up. The simulation is still a good approximation as it does keep the contrast reduction rate between stationary and rotational the same at around $50 \%$ speckle decrease. This allows the simulation to become a useful tool for preliminary design work with theoretical diffuser shapes.

As mentioned previously, the diffusers were illuminated with a coherent Gaussian source. While uniform illumination speckle statistics are generally considered proper, it is by no means correct as standard laser diodes will have some sort of Gaussian shell and any speckle created from such a diode will have statistics correlating to the 
Gaussian laser beam. All contrast values were measured without subtracting the Gaussian beam difference in the speckle to account for Gaussian shell noise in the speckle irradiance pattern. Verification of this assumption was completed using the simulation model and comparisons were made between uniform illumination and a Gaussian source. Minor differences can be seen within Figure 5.12 but the primary result of the Gaussian illumination is the slight spreading of the speckle pattern and lower peak intensity within the center of the image. Based on the speckle numbers, this result is trivial and evens out across the final image plane, as demonstrated through Figures 5.12a and 5.12b.

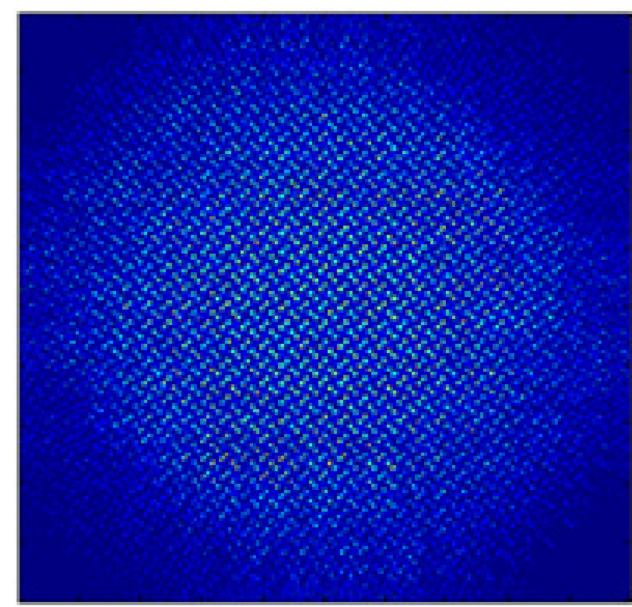

a)

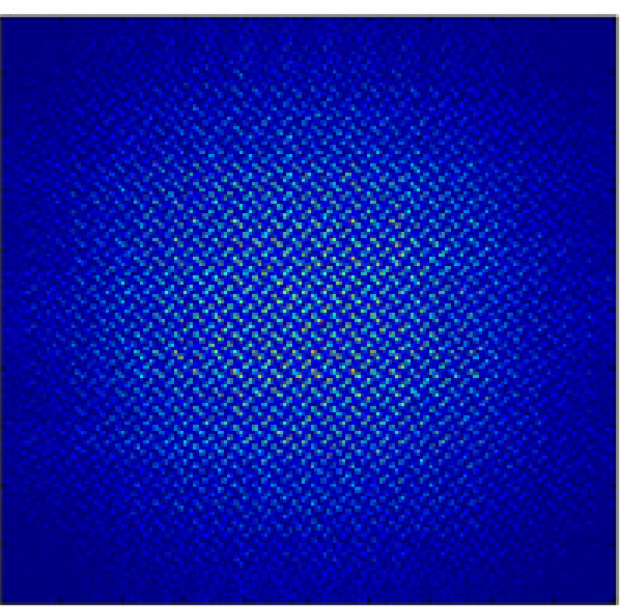

b)

Figure 5.12: (a) Diffuser model with uniform illumination; (b) Diffuser model with Gaussian illumination

\subsection{Diffuser Simulation Conclusion}

A Fourier optics based model was created to simulate and evaluate diffuser designs for speckle contrast reduction. The model was shown to be successful in mimicking physical field outputs for both stationary and rotating diffusers. The next step would be to incorporate projection optics to simulate the contrast reduction efficiency in 
pico-projector systems. The developed model was valuable in modeling and simulating novel diffuser designs. The desire is to create a new diffuser system that works without the need for moving parts as discussed in Chapter 3 and 4. In the following chapter a new diffuser design is theorized and thoroughly tested using the simulation method previously described. 


\section{Chapter 6: Simulation and Creation of New Diffuser Design}

\subsection{Hadamard Matrices for Speckle Reduction}

A new diffuser design for speckle contrast reduction in pico-projectors is required that alleviates the need of rotating or moving parts. As stated in the preceding chapters, moving parts are easily susceptible to mechanical failure, especially for parts in a hand-held device that is often dropped or crushed. Varying investigations into speckle reduction outside standard rotating diffusers have occurred $[13,14,16$, 35], but all require a moving element in the design. A more thorough listing of recent non-rotating or vibrating speckle reduction methods and the minimum achieved speckle contrast values are detailed in Table 1. As described in the table, other contrast reduction methods have resulted in low speckle, but all those methods revolve around some type of motion, even if the motion is not as extreme as high rpm rotation. In addition, the majority of speckle reduction methods require large setups or devices that would need to be contracted to accomplish a pico-projector like design. 
Table 6.1: Speckle contrast values for various speckle reduction methods

\begin{tabular}{lccr}
\hline $\begin{array}{c}\text { Author/Research } \\
\text { Group }\end{array}$ & $\begin{array}{c}\text { Speckle Contrast } \\
\text { (abs) }\end{array}$ & Contrast Reduction & $\begin{array}{r}\text { Reduction } \\
\text { Method }\end{array}$ \\
\hline $\begin{array}{l}\text { Ha, Lee, \& Jung } \\
\text { (Yonsei Unive.) }\end{array}$ & 0.18 & $70 \%$ & $\begin{array}{r}\text { Piezoelectric } \\
\text { Device } \\
\text { (Fiber Optics }\end{array}$ \\
$\begin{array}{l}\text { Tran, Subramaniam, } \\
\text { \& Le (Vestfold Univ.) }\end{array}$ & 0.48 & $44 \%$ & $\begin{array}{r}\text { MEMS Device } \\
\text { (Side-Side } \\
\text { Movement) }\end{array}$ \\
$\begin{array}{l}\text { Ouyang, Akram } \\
\text { (Vestfold Univ.) }\end{array}$ & 0.16 & - & $\begin{array}{r}\text { Piezoelectric } \\
\text { Device } \\
\text { (Angular }\end{array}$ \\
$\begin{array}{l}\text { Gao, Ma, \& Chen } \\
\text { (Beijing Institute) }\end{array}$ & 0.17 & $52 \%$ & $\begin{array}{r}\text { Stationary Diffuser } \\
\text { (Laser Scanning } \\
\text { Device) }\end{array}$ \\
\hline
\end{tabular}

A diffuser is investigated that has an associated phase shift of either 0 or $\pi$ for each individual phase element of the structure [44-46]. This corresponds to a common binary pattern of 0 or 1 . A Hadamard matrix is square matrix which is composed entirely of +1 and -1 values. It is used for performing Hadamard transforms (HT) [44]. The HT is a special generalized type of Fourier transforms and is accomplished through addition only, meaning that the output for this transform is produced through a summation of the individual Hadamard matrix components. This allows for faster computation compared to transforms requiring multiplication. A special property of the Hadamard matrix is the relationship between the matrix and its transpose as described in Equation 6.1. This definition requires all Hadamard matrices to be square with orders $\mathrm{n}=2,4,8,16$, where $H_{1}=[1]$ [44]. Multiple versions of the Hadamard matrix exist, each with a unique binary pattern. These unique patterns are further discussed in Appendix A. 


$$
H_{n} H_{n}^{T}=H_{n}^{T} H_{n}=n I_{n}
$$

The first type of Hadamard matrix is the Sylvester matrix and is described in

Equation 6.2 with the condition that $H_{1}=[1]$. An order 16 Sylvester matrix is shown in Figure 6.1 where the dark area denotes +1 and the white space denotes -1 .

$$
H_{2^{n}}=\left(\begin{array}{cc}
H_{2^{n-1}} & H_{2^{n-1}} \\
H_{2^{n-1}} & -H_{2^{n-1}}
\end{array}\right)
$$

The other Hadamard matrix types are known as a Walsh-Paley and a Walsh matrix, respectively, and can be constructed through recursion [44]. While each matrix still follows the basic definition, they all have an exclusive pattern creating different propagated patterns when observed. The -1 elements of the matrix can be swapped for 0 and a binary diffuser can be developed from the pattern.

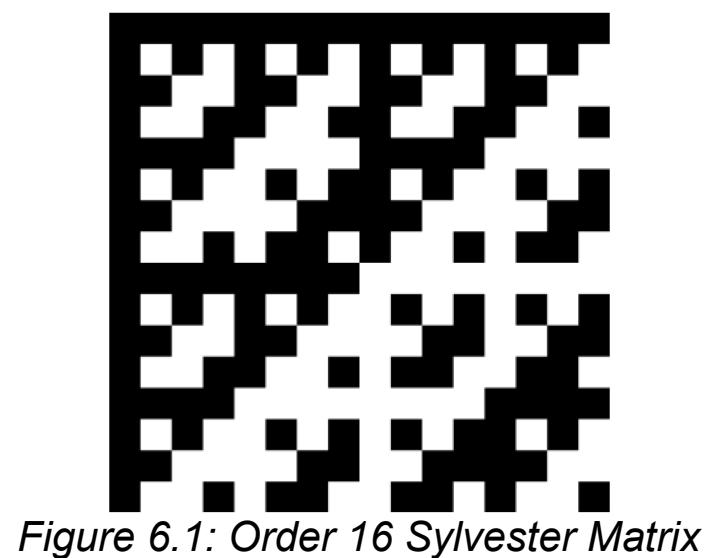

The measured speckle contrast for an initial rotating Hadamard diffuser design was 0.12 [45]. The original design requires upwards of 150-200 rpms for rotating diffusers 
in order to achieve speckle contrast at or below 0.20 as discussed previously in Chapters 1 and 2 regarding temporal averaging of a diffuser. A dual-inline diffuser based on Hadamard matrices is designed to reduce speckle while remaining completely stationary. Variations in the Hadamard matrix pattern types reduces speckle contrast without the need for temporal averaging. Using two statistically correlated Hadamard diffusers with varying phase patterns in tangent can reduce the speckle farther than two generic uncorrelated binary diffusers. The diffuser design was analyzed to determine whether the diffusers reduce speckle to the same level as a rotating diffuser. Another experiment attempting to design a stationary diffuser was conducted with binary code diffusers in 2012 where a single optimized binary diffuser was manipulated into two separate diffuser structures [14]. While stationary diffuser speckle was reduced, the method did not lower the contrast lower than previously demonstrated single rotating diffuser contrast values. The order numbers of the matrices chosen were not large enough to match the number of independent realizations achieved through rotation of the diffuser.

\subsection{Theoretical Analysis}

In order to theoretically verify the proposed dual Hadamard diffuser reduction technique, a single scattering, Fourier model is used for initial analysis and we will continue with a full system simulation using previous model techniques. A sixteen order matrix is created as an individual cell and duplicated to expand filling the total size of the array dependent on the analysis of the entire propagation system. For any

speckle reduction technique, Hadamard matrices included, a decrease by $(M)^{-1 / 2}$ can 
be achieved where $M$ is the degrees of freedom $[5,46]$. The degree of freedom represents the number of independent diffraction patterns that are created by a stationary diffuser $[11,12,34]$. A 16 order Hadamard matrix pattern can be expected to have a contrast measurement of 0.707 . Placing two differing Hadamard patterns in series along the optical path, within the Fresnel zone of the primary diffuser allows for a speckle value of 0.343 . Due to the correlation between the two patterns one half of the contrast reduction associated with the fully developed speckle can be assumed $[5,9]$.

The image irradiance level can be found by using Fourier propagation with the assumption that the illuminated diffuser speckle pattern will propagate onto a projection lens prior to observation onto a screen. This layout is illustrated in Figure 6.2 where $z_{1}$ and $z_{2}$ represent the propagation distances between the diffuser, lens, and observation screen, where $f$ represents the focal length of the lens. Initial analysis will involve a single diffuser to verify the approach to satisfy the previously expected contrast values.

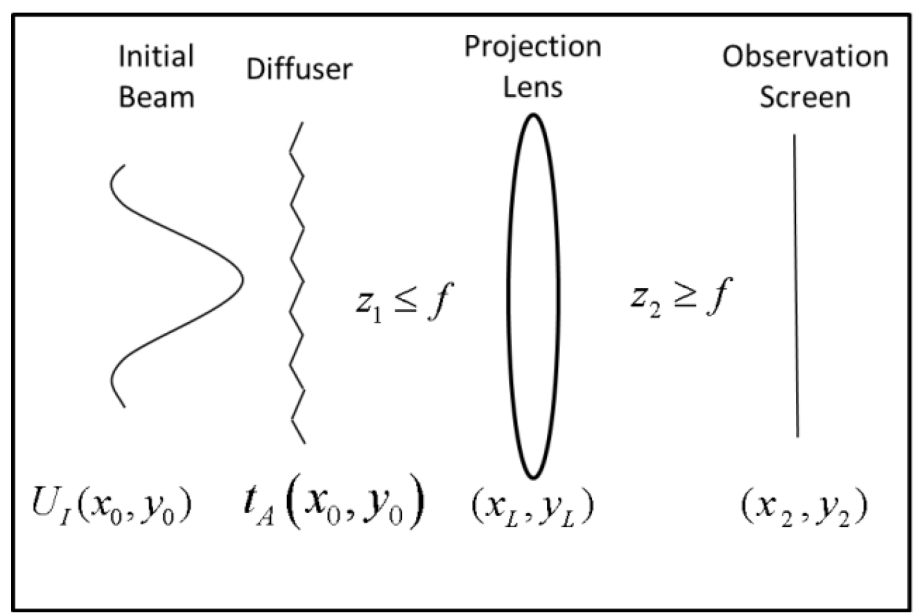

Figure 6.2: Current diffuser layout using projection lens 
The initial propagation prior to incidence on the diffuser as coherent monochromatic Gaussian light is defined by Equation 6.3.

$$
U_{G}(x, y)=A_{G} e^{\frac{x^{2}+y^{2}}{w_{0}^{2}}}
$$

$A_{G}$ represents the amplitude of the beam and $w_{0}$ is the initial waist of the beam. The diffuser can be modeled as a single scattering phase screen with the Hadamard matrix structure defined in Section 5.2, Equation 5.1. This matrix can be replicated to represent the whole of the diffuser as described in Equation 6.4.

$$
t_{A}(x, y)=e^{j \pi / 2\left[\begin{array}{ccc}
H_{16} & \ldots & H_{16} \\
\ldots & \ldots & \ldots \\
H_{16} & \ldots & H_{16}
\end{array}\right]}
$$

The $\frac{\pi}{2}$ in the exponential, is the phase shift that separates the two levels of the diffusers. The incident illumination can be multiplied by the diffuser within the Fourier domain since it is modeled as a phase screen. It is assumed that the Gaussian illumination is a negligibly small distance from the incident diffuser which allows direct convolution of the incident beam and the diffuser. The initial optical field immediately after the diffuser is defined in Equation 6.5.

$$
U_{I n c}\left(\xi_{I n c}, \eta_{I n c}\right)=\mathfrak{J}\left\{U_{G}\left(x_{I n c}, y_{I n c}\right) * t_{A}\left(x_{I n c}, y_{I n c}\right)\right\}
$$

In Equation 6.5, $\mathfrak{I}\{\ldots\}$, represents a Fourier transform from real space into the frequency domain. Propagation after the diffuser can be accomplished through the 
transfer matrix approach [46], taking into account the distance of propagation is still within the near-field region. Equation 6.6 shows the result of propagation after the diffuser.

$$
U_{D}\left(x_{D}, y_{D}\right)=\frac{e^{j k z}}{j \lambda z} \iint U_{I n c}(\xi, \eta) e^{j \frac{k}{2 z}\left[(x-\xi)^{2}+(y-\eta)^{2}\right]} d \xi d \eta
$$

Due to the very small size of the diffuser elements in $x$ and $y$, the phase propagated pattern size will increase rapidly along the $z$ axis causing the lens to be well within the focal length and thus within the Fresnel region of the diffuser output. This satisfies the condition for Fresnel approximation given by Equation 6.7 [21].

$$
z^{3}>>\frac{\pi}{4 \lambda}\left[(x-\xi)^{2}+(y-\eta)^{2}\right]_{\max }^{2}
$$

An important element in measuring the speckle is to ensure the lens does not decrease the size of the speckle spots so far that the individual pixels of the camera used for measurement does not cause averaging, independent of the rest of the system [26]. This "matching" of lens to speckle screen was used extensively for measuring laser beam size affects and diffuser speed while maintaining the individual speckle spot size, effectively comparing the speckle contrast across the surface [34]. A lens, $L$, can be described as a phase function applied to the incoming wave. The transmittance function of the projection lens is described by Equation 6.8 [21].

$$
t_{L}\left(x_{L}, y_{L}\right)=P\left(x_{L}, y_{L}\right) e^{\left[-j \frac{k}{f}\left(x_{L}^{2}+y_{L}^{2}\right)\right]}
$$


For a lens function it can be shown that the distance $d$ is replaced by the focal length of the lens $f$. The aperture function $P$ can be simply described as a circle $[21,36]$. Propagation of the lens can occur by using the Fraunhofer propagation equation and replacing the distance parameter $z$ with the focal length $f$, of the lens [36]. The generic Fraunhofer equation is described in Equation 6.9. Substituting the results from Equations 6.6 and 6.8 into Equation 6.9 resolves into the expression shown in Equation 6.10.

$$
\begin{gathered}
U_{f}\left(x_{f}, y_{f}\right)=\frac{\mathrm{e}^{j k z}}{j \lambda z} e^{\left[j \frac{k}{2 z}\left(x^{2}+y^{2}\right)\right]} \iint U_{1}(\xi, \eta) e^{\left[-\frac{2 \pi}{\lambda z}(x \xi+y \eta)\right]} d \xi d \eta \\
\left.U_{f}\left(x_{f}, y_{f}\right)=\frac{e^{j k f}}{j \lambda f} e^{\left[j \frac{k}{2 f}\left(x_{f}^{2}+y_{f}\right)\right]}\right] \int U_{D}\left(x_{D}, y_{D}\right) P\left(x_{f}, y_{f}\right) e^{\left[-\frac{2 \pi}{\lambda f}\left(x_{f} x_{D}+y_{f} y_{D}\right)\right]} d x_{D} d y_{D}
\end{gathered}
$$

$U_{f}$ represents the amplitude of the wave of the resultant propagation at the focal plane of the lens $L$.

After the focal plane of the lens, propagation to the observation plane can be evaluated using the Fresnel propagation equation described previously by Equation 6.6. The final amplitude output of the system $U_{\text {obs }}$ can be described by Equation 6.11 where Equation 6.10 can be viewed as the initial amplitude field and is propagated using the Fresnel approximation to the final observation screen. 


$$
\begin{aligned}
& U_{O b s}\left(x_{O b s}, y_{O b s}\right)=\frac{e^{j k z_{2}}}{j \lambda z_{2}} \iint \frac{e^{j k f}}{j \lambda f} e^{\left[\frac{j k \frac{k}{2 f}\left(x_{f}^{2}+y_{f}^{2}\right)}{}\right.} \iint U_{D}\left(x_{D}, y_{D}\right) \\
& \times P\left(x_{f}, y_{f}\right) e^{\left[-j \frac{2 \pi}{\lambda f}\left(x_{f} x_{D}+y_{f} y_{D}\right)\right]} d x_{D} d y_{D} e^{j \frac{k}{2 z}\left[(x-\xi)^{2}+(y-\eta)^{2}\right]} d \xi d \eta
\end{aligned}
$$

The distance from the focal plane of the lens to the final observation plane is greater than previously described for the diffuser to the lens. This distance $z_{2}$ is still well within the near-field distance requirement and satisfies Equation 6.7. Far-field propagation will need to be considered for projection onto surfaces for image viewing (actual projector system), but is not applicable for the detection measurement $[5,26]$.

Contrast measurements for the output speckle images can now be found from the previously discussed contrast equation involving speckle image intensity as described in Equation 3.2. The intensity of the observation measurement from the optical field in the observation plane and can be broken into pixel elements as shown in Equation 6.12.

$$
I_{2}\left(x_{2}, y_{2}\right)=\left|U_{2}\left(x_{2}, y_{2}\right)\right|^{2}
$$

Thus, the overall contrast created by the Hadamard diffuser can be attributed to taking multiple Fourier transforms of the initial phase screen $t_{A}$. A second phase screen can now also be included by evaluating Equation 6.5 again with all elements the same except for the phase screen. The analysis for both diffusers will need to maintain the same $z$ value associated with the diffusers prior to the lens to maintain Fresnel region 
propagation. A simulation will be used to further the previous analysis and provide an accurate accounting of the contrast reduction provided by the diffuser. Modeling of the $z$ value to match the phase elements of both diffusers will be discussed in the next section.

\subsection{Hadamard Simulation}

An initial modeling of the primary diffuser is accomplished first to ensure the proper contrast evaluation prior to a two diffuser simulation [47]. Both contrast values will be compared with the expected theoretical values for accuracy. Fourier analysis requires that the discrete values associated with the sampling of the arrays be properly related to the linear size of each array. In this situation the two primary physical objects that require extended examination are the diffuser, or diffusers, and the lens. A lens is used in speckle imaging and the aperture of the lens will determine the final speckle spot size in the observation plane [5]. This configuration is known as subjective speckle. An important part of measuring the speckle contrast is ensuring that the speckle spots will not be averaged together in a single pixel of the observation screen [26]. This will be fixed by ensuring a large array for the lens that will project the pattern propagated from the diffuser. An 8000 x 8000 array group will be used for each screen, i.e. Gaussian input, diffuser, and lens. This means a sampling size of $3.89 \mu \mathrm{m}$ per pixel given a $54.8 \mathrm{~cm}$ diameter lens and a $25.4 \mathrm{~cm}$ linear, square diffuser. The most advanced photolithography machines are capable of incrementing diffuser size of around $1 \mu \mathrm{m}$. This physical size limitation is well within the current standards for producing an actual diffuser and our simulated sampling size stands. 
The final output for the single Hadamard diffuser propagated through a projection

lens is shown below in Figure 6.3. The output directly after the diffuser and a smaller cropped version of the final output image are shown as well.

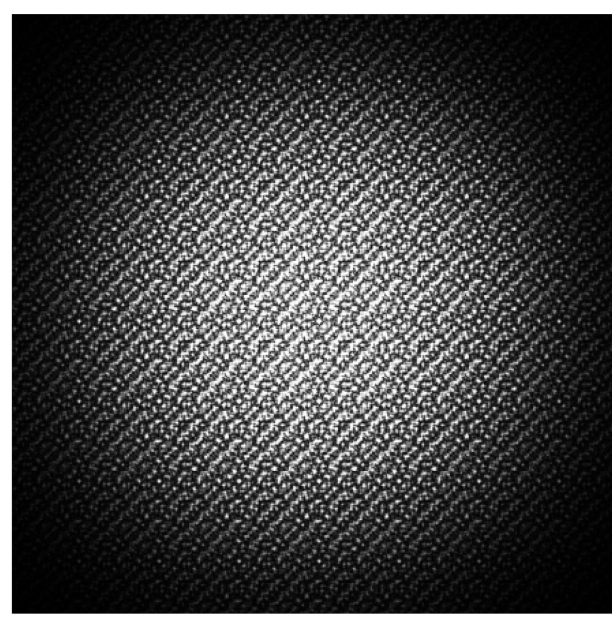

a)

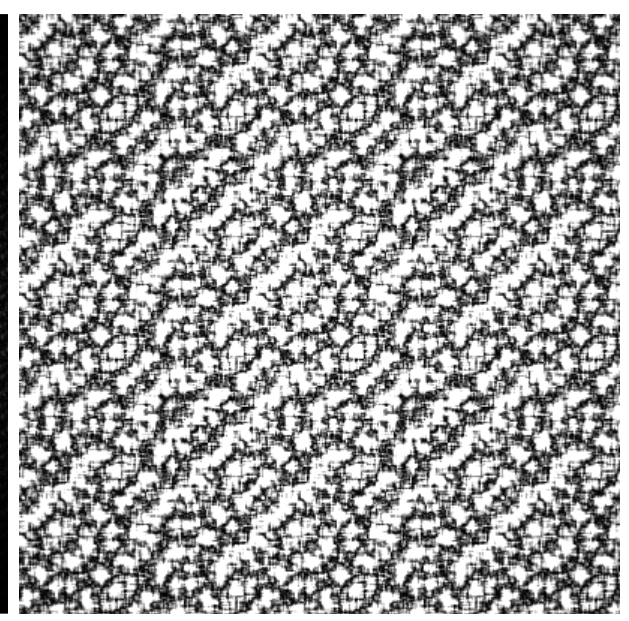

b)

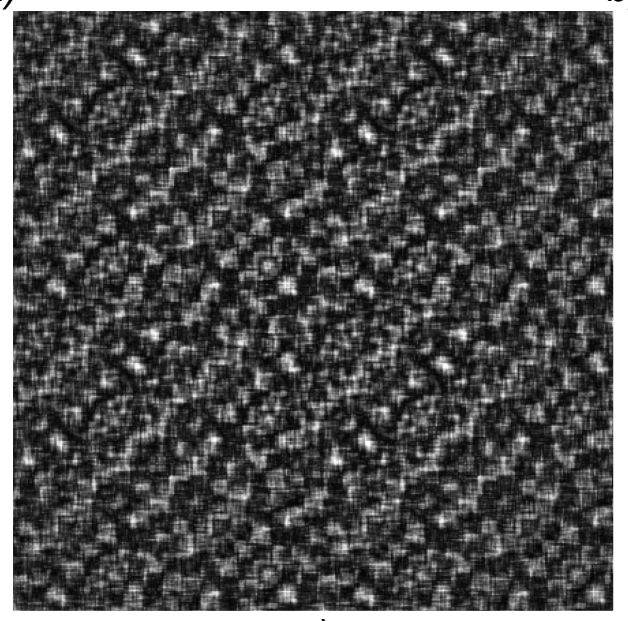

c)

Figure 6.3: Single diffuser simulation results, a) Intermediate Image Screen, b) Final Image Screen, c) Final Image normalized

The contrast provided by the final image is $0.67+/-0.02$, which is comparable to our accepted theoretical value of 0.707 for a single Hadamard diffuser [45]. A two diffuser output image is shown next in Figure 6.4. The distance between the two diffusers was set at $2 \mathrm{~mm}$. The speckle contrasted provided by the final image is 0.43 $+/-0.02$. The theoretical limit was calculated to be 0.343 . 
The most plausible reason behind the difference in speckle contrast between the theoretical and the simulation is the lack of polarization associated with the propagated light. A bench top experiment using these diffusers will be able to analyze the polarization dependence of the speckle contrast in the diffuser and provide a more accurate contrast measurement. A possible improvement to the current model would be to include polarization in the initial incident beam. This would also require that each surface be able to alter the polarization correctly for that material type i.e. glass for diffuser or lens. 


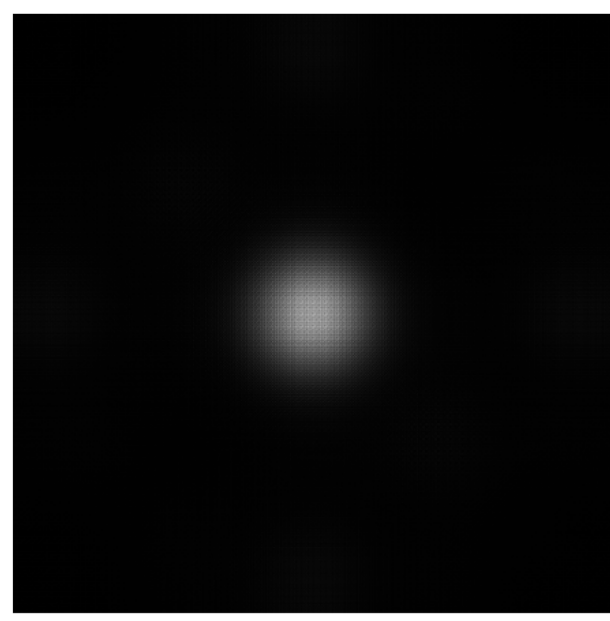

a)

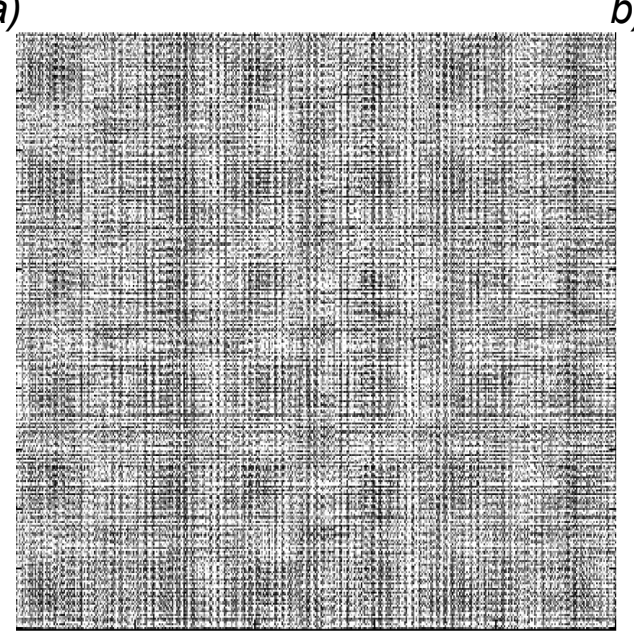

c)

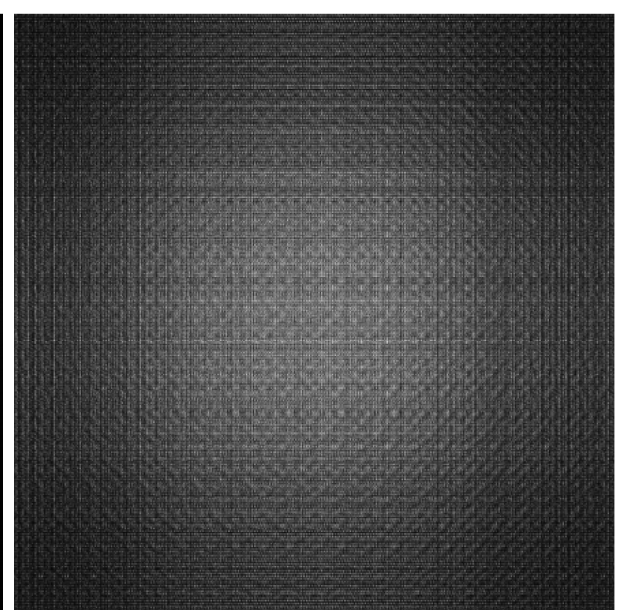

b)

Figure 6.4: Dual diffuser simulation results, a) Image directly after second diffuser, b) Final image output from dual diffuser system, c) Close up image output from dual diffuser system

\subsection{Experimental Results and Comparison}

Prototype versions of the designed diffuser were manufactured by Jenoptik, Microoptics Division(Formerly MEMS Optical) ${ }^{2}$. Speckle contrast results were measured for the diffusers using a $532.8 \mathrm{~nm}$ DPSS laser with pseudo-random linear polarization. The laser was spatial filtered prior to incidence on the diffuser. A single diffuser was tested first with a speckle contrast minimum of 0.62 and an average of

${ }^{2} 205$ Import Circle, Huntsville, AL 35806 (256) 859-1886 
$0.74(+/-0.02)$ using a $25 \mathrm{~mm}$ focal length projection lens. A Thorlabs CCD camera was used for image capture with a resolution of 480x640 pixels and a pixel size of 7 $\mu \mathrm{m}$ squared. Figure 6.5 illustrates the output results that were used for measurement. The image results from the single physical Hadamard diffuser matches the output results of a zoomed-in version of the simulation output in Figure 6.3(c).

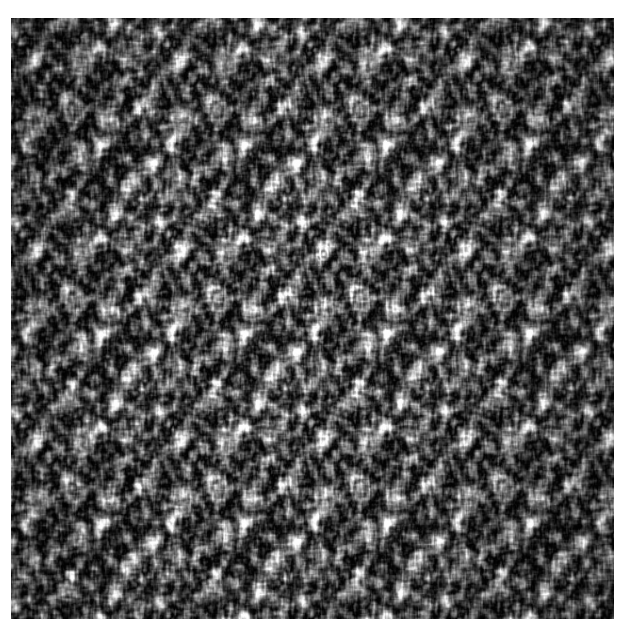

Figure 6.5: Physical Hadamard diffuser - image capture

Two diffusers were setup in series and the contrast results were measured. The projection lens was again a $25 \mathrm{~mm}$ focal length positive lens. Final contrast results for dual Hadamard diffusers were slightly more difficult to fully appreciate due to mitigating factors such spatial alignment, separation distance between diffusers, and speckle spot size. Each of these factors can arguably affect the speckle output so all conditions of testing must be fully analyzed for each contrast result. A standard separation distance between the diffuser of approximately $5 \mathrm{~mm}(+/-0.5 \mathrm{~mm})$ was setup. A minimum speckle contrast of 0.40 was achieved. There was some variance in the speckle measurements of about $8-12 \%$ change in the contrast being possible. This contrast variance was much higher than expected, but it results from the lack of 
optimization with the separation distance between the two diffusers. Simulation results confirm that separation distance between the diffusers can have a significant impact on the resulting speckle contrast value which was subsequently confirmed with the physical measurements fluctuations. Further research will be completed to optimize this particular distance to enhance the speckle reduction capacity of the Hadamard matrix diffuser set. Figure 6.6 illustrates the output from a dual diffuser setup.

The minimum value achieved was within $2-3 \%$ of expected simulation results although it was still greater than $6 \%$ than theoretically predicted. It is hypothesized that a possible difference in contrast is due to larger than anticipated Gaussian beam affects and pixel to speckle spot size matching [26]. While the simulation attempts to overcome pixel size mismatch by utilizing large array sizes, the camera available for image capture has only a pixel size of 7 microns squared. This is still of minor importance as differences in theoretical versus physical measurements are within the variance caused by the distance discrepancies of the two diffusers.

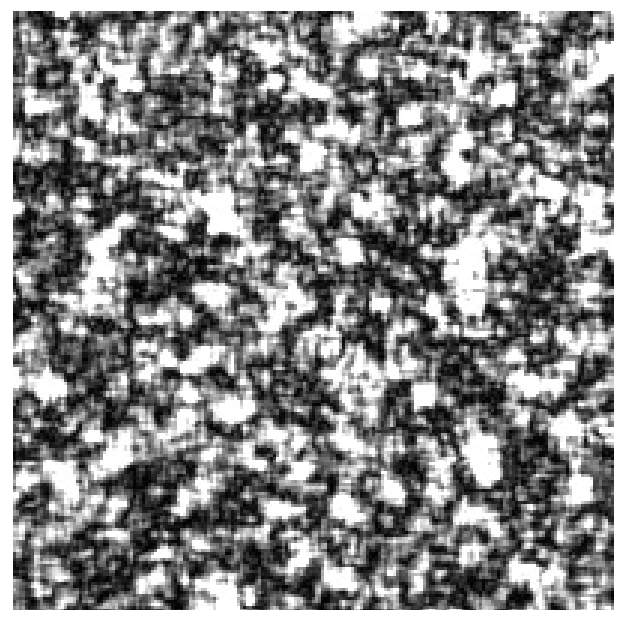

Figure 6.6: Dual Hadamard diffusers - image capture 
The effects of linear polarization were evaluated with the physical diffuser set.

Investigations discussed in Chapter 2 show that polarization has little to no impact on rotating diffusers [34]. A polarizer was implemented prior to the laser's incidence on the diffuser. Speckle contrast was measured for various linear polarization positions. The contrast measured from polarized images was $0.46(+/-0.02)$. This is a slightly higher speckle contrast than with random polarization. However, this change is within error parameters caused by the physical setup of the diffuser so it is of interest only in situations where the laser is completely polarized.

\subsection{Dual Hadamard Diffuser Conclusion}

A static, dual in-line Hadamard diffuser optical system has been demonstrated for speckle reduction in pico-projection applications. Hadamard diffusers were fabricated and measured with good agreement between experimental contrast measurements and simulated values. A minimum contrast of $0.40(+/-0.08)$ was achieved for monochromatic illumination without using rotation or vibrational movements. The diffuser design required only two distinct optical sub-systems: the diffusers and the projection optics. The dual-diffuser design had a speckle contrast value comparable to other speckle reduction methods but had significantly less complexity and is specifically designed for pico-projector devices. 


\section{Chapter 7: RGB Application of Hadamard Diffusers}

\subsection{Polychromatic Speckle Introduction}

In order to fully utilize diffusers in projector applications, polychromatic operation must be examined. Speckle contrast viewed from overlapping polychromatic images is analyzed in this chapter. Most digital displays - including televisions and computer monitors - use some variation of only red, green, and blue colors for image projection. The results of this research focus on those three wavelengths. This chapter investigates using Hadamard matrix diffusers (DHDs) for contrast reduction in polychromatic speckle. Analysis of the DHDs ability to decrease speckle for each wavelength at 635,535 and $450 \mathrm{~nm}$ and in combination of all three wavelengths is discussed. The research described here also investigates the polychromatic laser setup needed to measure the speckle contrast resulting from DHDs. Full simulation and experimental results from these comprehensive analyses is presented. Finally, the distance of separation between the DHDs has been examined to determine the relationship between speckle contrast and spatial movement between two diffractive optical elements. Each wavelength and full color speckle will be measured across varying distances between simulated DHD. 


\subsection{Wavelength in Diffractive Optics}

Previous work has been completed simulating DHDs for speckle reduction at $535 \mathrm{~nm}$ green laser light $[47,48]$. Speckle contrast of $0.43(+/-0.02)$ was achieved at the single wavelength. Diffusers were manufactured based on this simulated design and tested using a DPSS green laser. The simulated and actual speckle images from the DHD are shown side-by-side in Figure 7.1. Physical bench top testing resulted in a minimum speckle contrast measurement of $0.40(+/-0.08)$ [48]. While the speckle reduction is not as low as other proposed reduction systems, the DHD do not require any rotation or vibration creating a completely stationary subsystem ideal for handheld electronic devices.

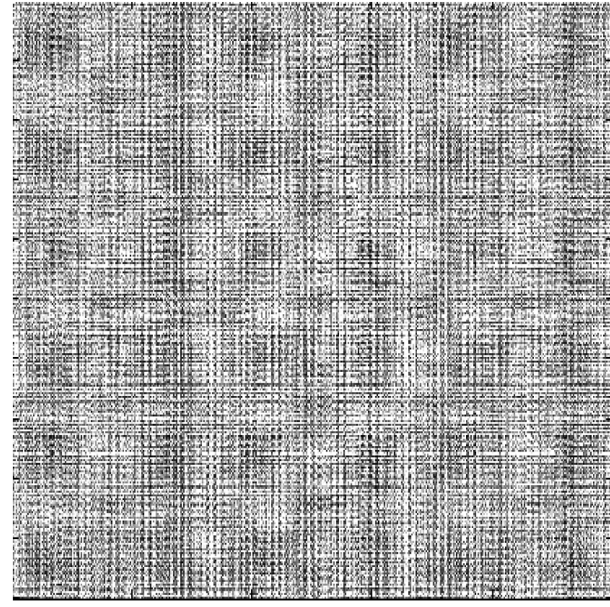

a)

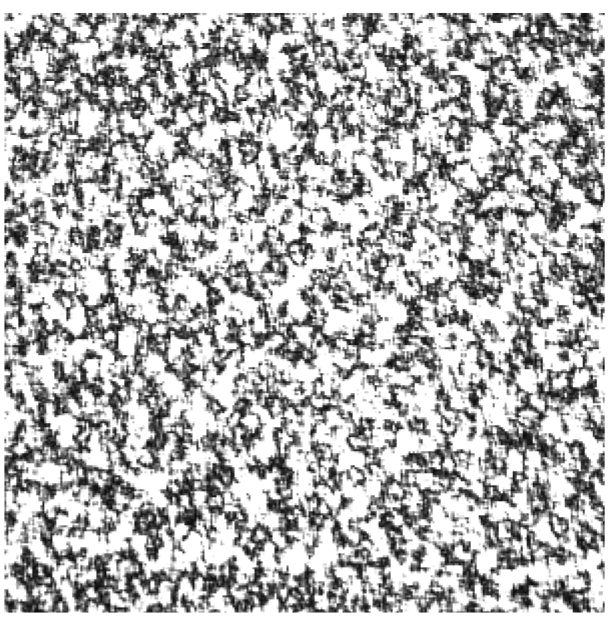

b)

Figure 7.1: Simulated (left) and experimental (right) speckle images from DHDs with $535 \mathrm{~nm}$ laser light

Diffractive diffusers can be designed to have up to 32 varying phase levels, but all diffractive optics can still be described by the grating equation. The grating equation is shown in Equation 7.1 and describes diffracted light normally incident on the diffuser [8]. 


$$
d \sin (\theta)=m \lambda
$$

Making element size $d$ and order number $m$ constant, Equation 7.1 can be re-arranged to show a direct relationship between diffracted angle, $\Theta$, and wavelength as shown in Equation 7.2 (incident light is normal to the grating surface) [8].

$$
\theta(\lambda)=\sin ^{-1}\left(\frac{m \lambda}{d}\right)
$$

As the wavelength of incident light increases or decreases, the angle of expansion for the orders increases and decreases as well. The angular dispersion of a diffractive grating can be found by taking the derivation of the grating equation, Equation 7.1, with respect to the wavelength as described in Equation 7.3.

$$
\frac{\partial \theta}{\partial \lambda}=\frac{m}{d \cos (\theta)}
$$

For contrast reduction purposes, the defined angular dispersion means the speckle spots will separate at a greater angle for red at $635 \mathrm{~nm}$ compared with blue at $450 \mathrm{~nm}$. This is important to realize when attempting to combine red, green, and blue and reduce speckle across each wavelength using a single optical element. This effect is demonstrated in Figure 7.2 with the simulated output of a DHD set at three wavelengths. 
As the wavelength decreases from Figure $7.2 \mathrm{a}$ at $635 \mathrm{~nm}$ to Figure $7.2 \mathrm{c}$ at $450 \mathrm{~nm}$ the speckle is seemingly contracted. The consequence of the relationship between diffraction angle and wavelength is that the three speckle images combined do not have overlapping speckle spots. The eye perceives an effective smoothing as dark pixels are eliminated and speckle contrast is reduced. This effect can be observed in Figure 7.2d where speckle images are simulated using DHD and then combined.

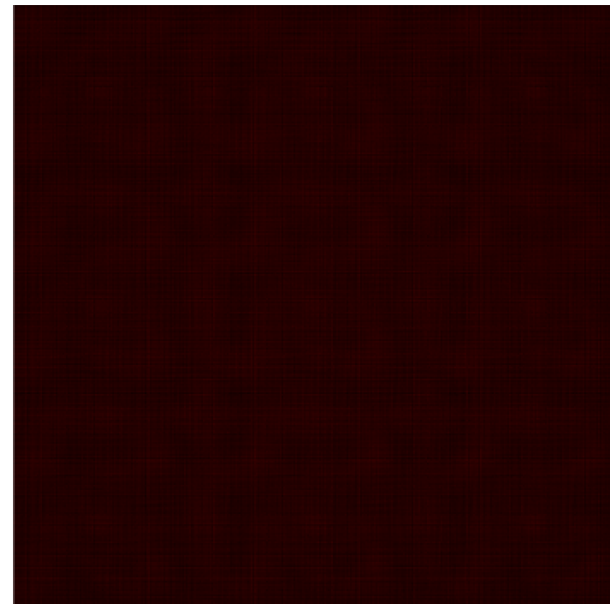

a)

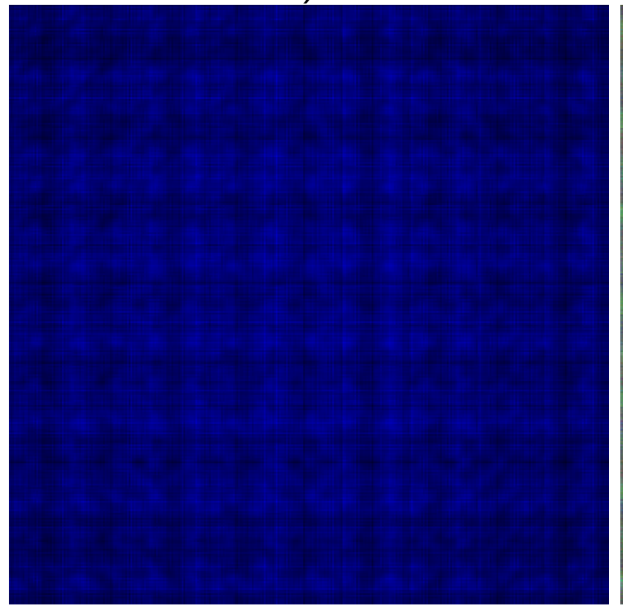

c)

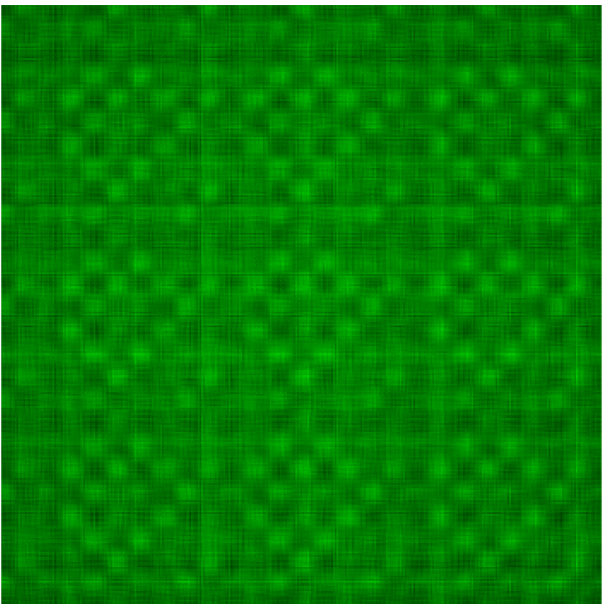

b)

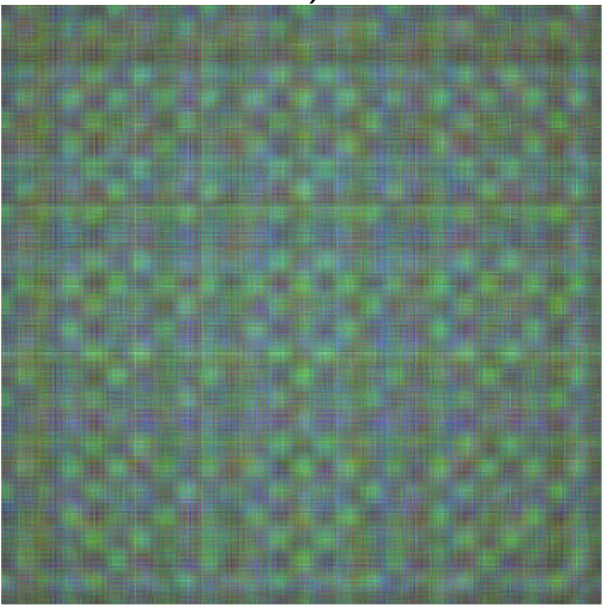

d)

Figure 7.2: Simulated dual Hadamard diffusers at a) $635 \mathrm{~nm}$, b) $535 \mathrm{~nm}, \mathrm{c}$ ) $450 \mathrm{~nm}$, d) 635,535 , and $450 \mathrm{~nm}$ overlaid together 


\subsection{Measuring RGB Speckle Contrast Images}

There are two possible methods to setup diffusers for operation in an RGB laser setup. The diffusers can be placed after the lasers have been combined and aligned together and then focused with a single projection lens. This setup is shown in Figure 7.3a. A consequence of this is that the phase shift is not consistent across all three wavelengths. A second setup is to have a separate diffuser(s) for each laser prior to combination and alignment. This allows you to optimize the phase shift specifically for each wavelength, but space needs are increased with multiple diffusers. In addition, it is possible that different projection lenses may be required to keep the output speckle image consistent in size for each wavelength. This condition is rather complicated because it is completely dependent on how each individual laser is positioned in comparison to one another. An example setup is shown in Figure 7.3b. This chapter examines both setups, but focuses more on the single diffuser setup for all wavelengths due to time constraints and costliness of designing and manufacturing multiple diffusers.

Multi-wavelength speckle contrast was measured by utilizing the previously developed Fourier optics based simulation to propagate the laser through the diffuser onto a detector (See Chapters 5 and 6). Theoretical calculations for monochromatic DHD result in a contrast of 0.35 as per Section 6.4. Differing wavelengths do not interfere with one another. Introducing two additional non-interfering speckle patterns from DHD (two diffusers) adds 8 additional degrees of freedom resulting in a total speckle reduction of 0.13 [5]. However, color detectors, including the eye, do not 
capture differing wavelengths across a single pixel, but separate into red, green, and blue. Speckle contrast is then measured by the average across the three separate intensity images. This would actually produce a theoretical speckle contrast of 0.11 . Grayscale detectors will measure multiple colors across a single pixel while adding together in amplitude. This creates a single contrast pattern that can be measured. Unity normalization is needed to ensure both simulated detection output images can be compared.

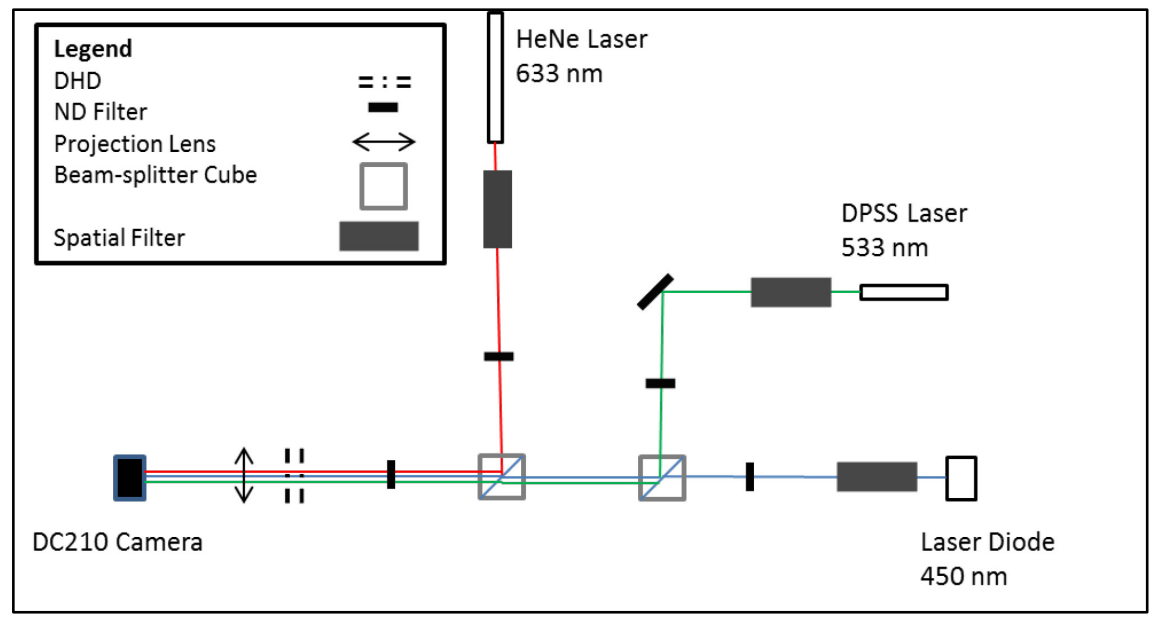

a)

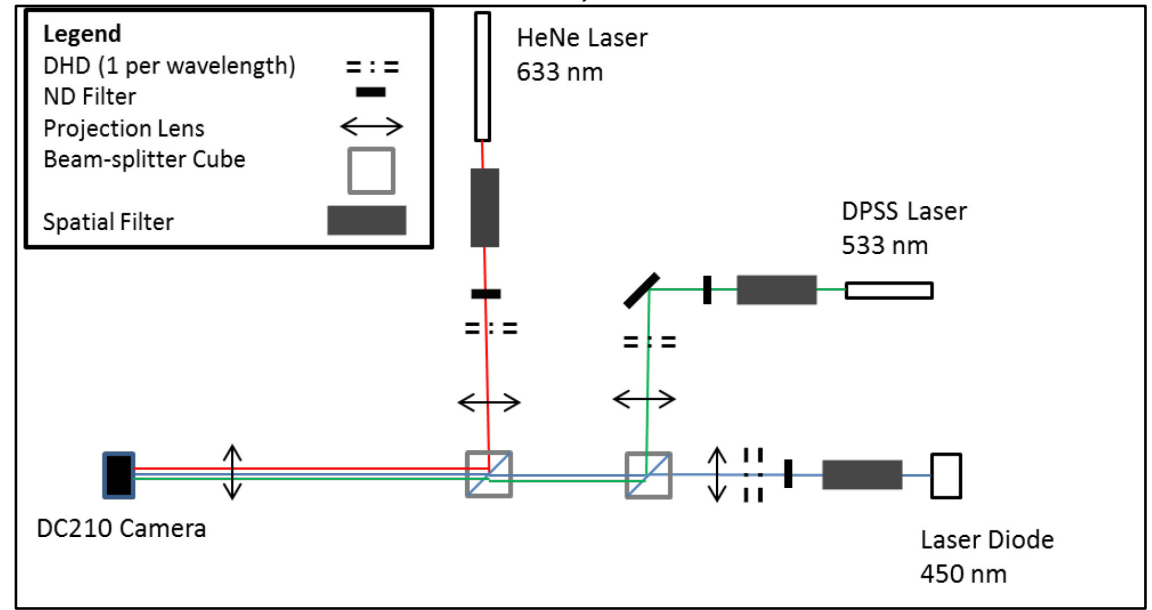

b)

Figure 7.3: Multi-wavelength speckle setup using a) a single DHD b) multiple $D H D$ 
Each detection method was simulated for full color speckle patterns in order to compare them to bench top results which used a grayscale camera for image capture. Laser wavelengths of $635 \mathrm{~nm}, 535 \mathrm{~nm}$, and $450 \mathrm{~nm}$ were used to coincide with common laser wavelengths. Grayscale detection resulted in a speckle contrast of 0.19 , while color detection resulted in a speckle contrast measurement of 0.18 . Figure 7.4 demonstrates the output results for each of the detection methods, as well as the averaged RGB image.

These measurement results correspond with pi/2 phase changes at all three wavelengths, corresponding with the setup in Figure 7.3b. Physical bench top results more in line with the setup in Figure 7.3a will be utilized and must account for only a single physical diffuser. An etched diffuser will have fixed phase levels based on Equation 7.4 [49].

$$
\Delta \phi=\frac{2 \pi(n-1) d}{\lambda}
$$

For fused silica $n$ is 0.4548 at $535 \mathrm{~nm}$, the wavelength, $\lambda$ with a known phase difference $d$ the etched phase level depth can be determined. This fixed value will correspond to differing phase values at differing wavelengths. There is then a slight phase change difference at each wavelength incident on the diffuser. In this situation, there is a + pi/10 phase shift at $635 \mathrm{~nm}$ and a -pi/10 phase shift at 450 . This effect can be adjusted for in the simulation by simply altering the phase screen of propagation for each wavelength. This results in speckle contrast measurement of 0.24 and 0.22 
for grayscale and color detection, respectively. Although slight, the phase adjustments do have an impact on the final speckle contrast since previous simulations without phase adjustments achieved a contrast of 0.18 . The speckle contrast values adjusted for phase drift are used for comparison with the bench top results.

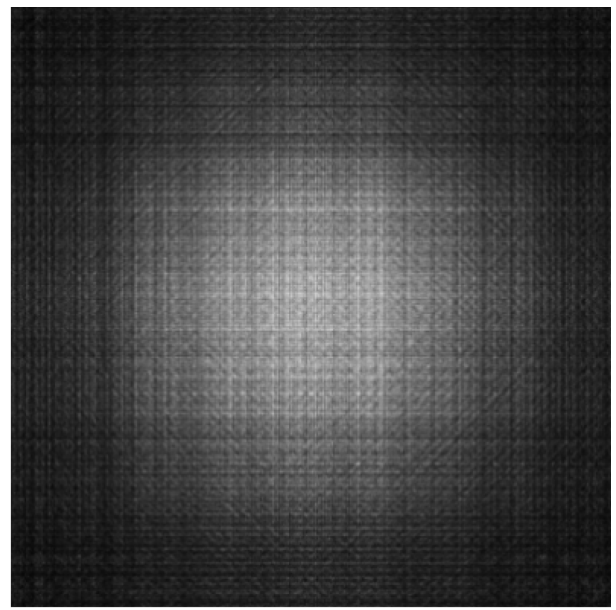

a)

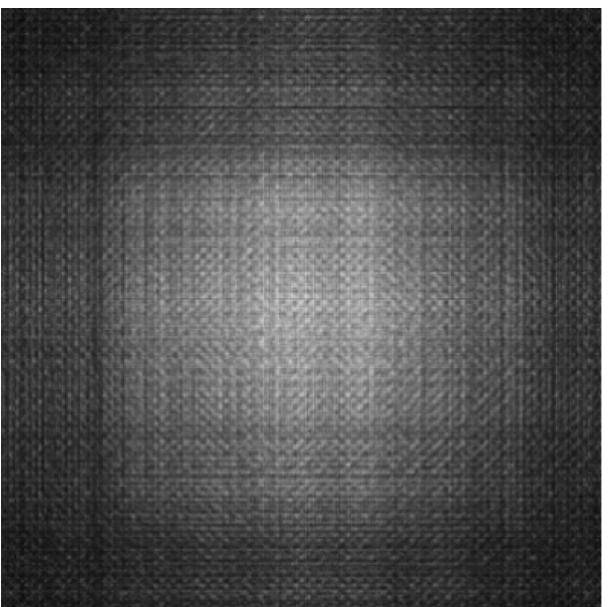

b)

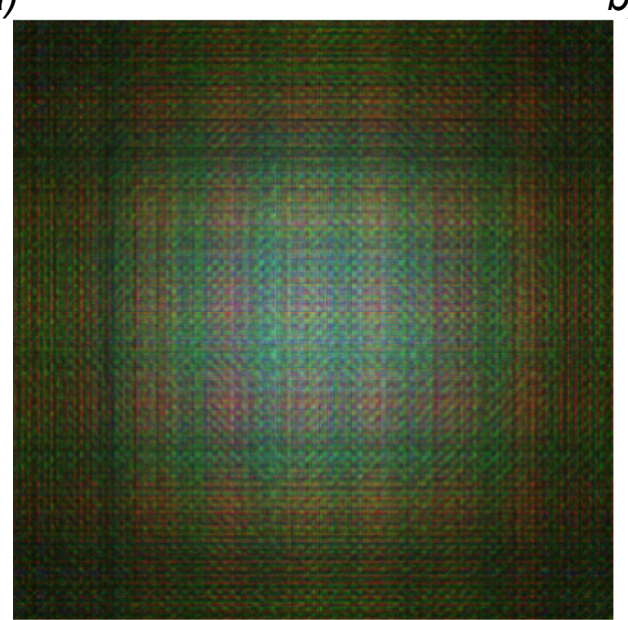

c)

Figure 7.4: Simulated RGB DHD in a) grayscale, b) averaged RGB image, c) full color RGB image

Bench top results for a three laser speckle image were analyzed for speckle contrast reduction. Figure 7.5 shows the bench top optical setup. All three lasers maintain the same beam size and intensity at incidence with the diffuser. A Thorlabs DC210 CCD grayscale camera was used to capture the speckle images with a frame rate of $30 \mathrm{~Hz}$ 98 
and a resolution of $640 \times 480$ ( $7 \mu \mathrm{m}$ pixels). The spectral response of the camera was examined in order to keep the irradiances equal across all three wavelengths. The spectral response curve of the DC210 is depicted in Figure 7.6. The responsivity of the CCD detector at 450 and $635 \mathrm{~nm}$ is $30 \%$ less than the response of the detector at $535 \mathrm{~nm}$. The power levels of the laser were adjusted to ensure measurement accuracy. A speckle contrast value of $0.25(+/-0.10)$ was measured. A large variance occurred in the experimental speckle measurements due to having three individually aligned lasers. Slight modification to each of the laser setups resulted in a change in speckle and together created a larger error value than previously measured. Figure 7.7a shows the final grayscale bench top image captured with Figure $7.7 \mathrm{~b}$ showing the same bench top image except with RGB detection. 


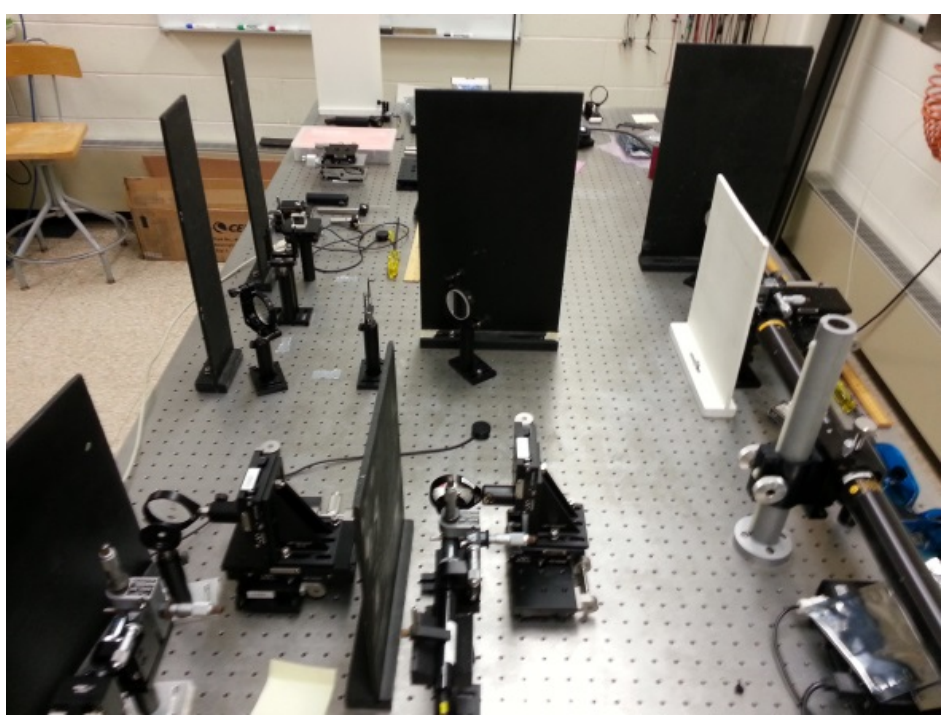

a)

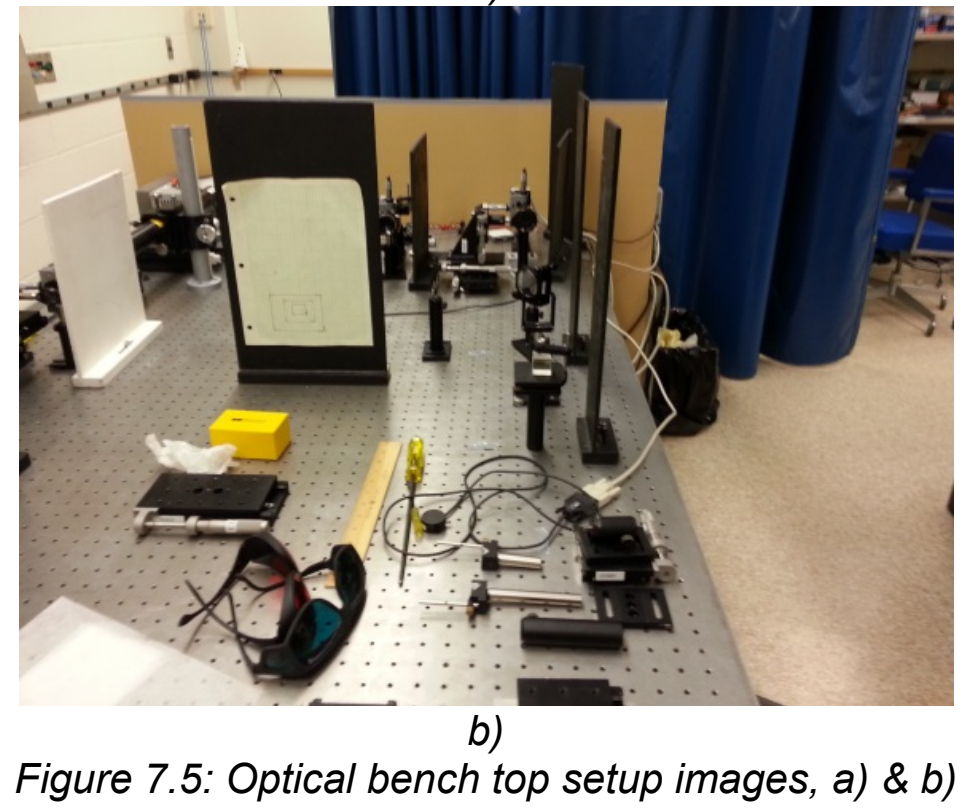




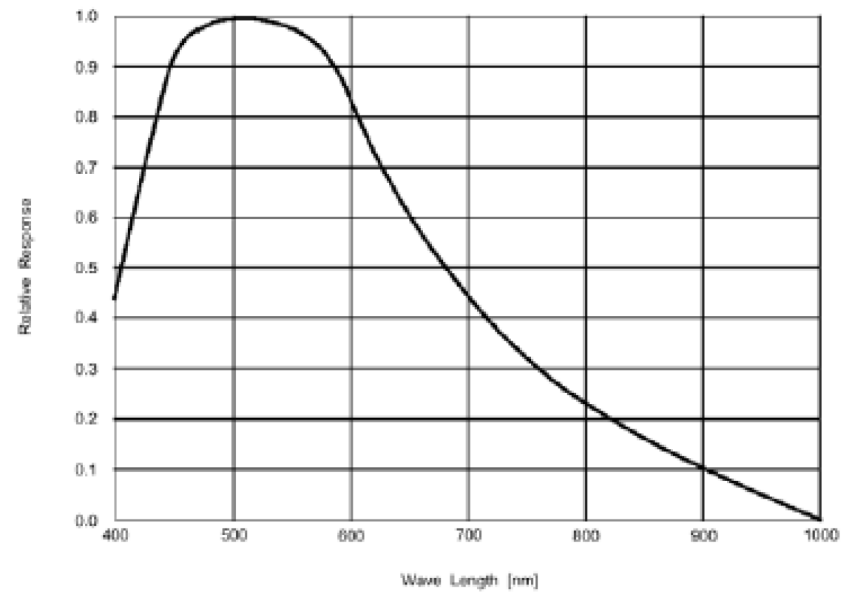

Figure 7.6: Spectral response curve for DC210 (Thorlabs)

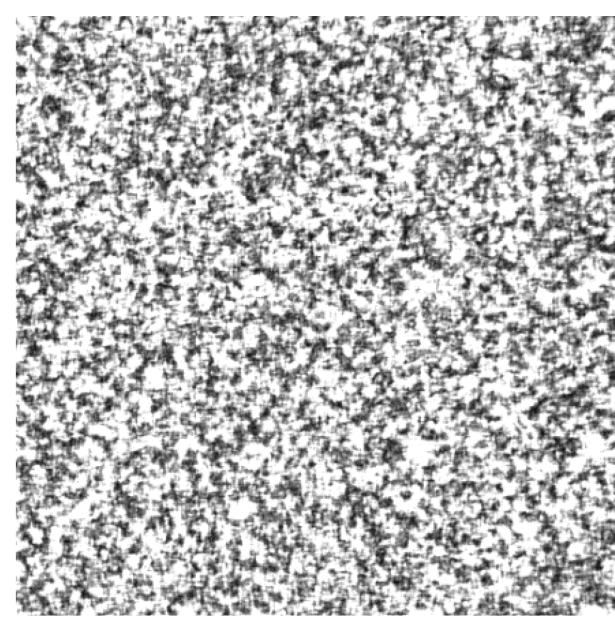

a)

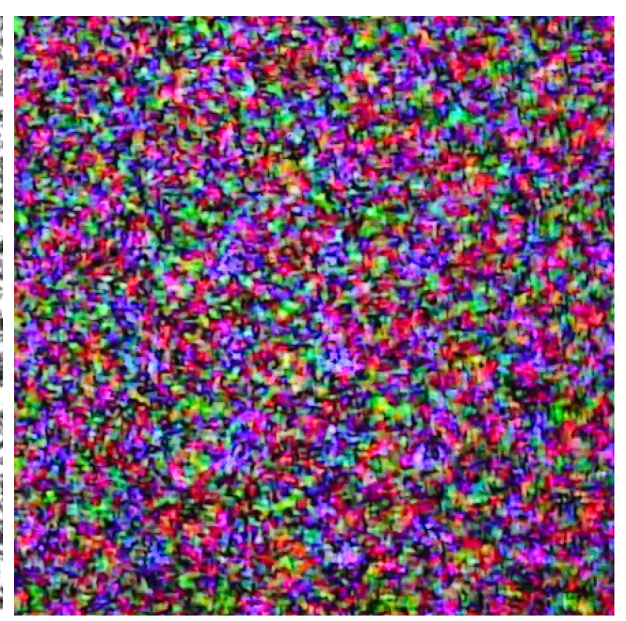

b)

Figure 7.7: Bench top speckle image using a) grayscale detection and b) $R G B$ detection

\subsection{Diffusers Separation Distance}

Speckle contrast values were $10 \%$ above the expected theoretical values. This can be accounted for by altering the separation distance between the two diffusers.

Simulations conducted analyzed how the separation between the diffusers affects the speckle contrast. Separation distance of the DHD were compared across the three wavelengths separately and with all three wavelengths combined using both grayscale 
and color detection. The individual wavelength results were simulated assuming a single DHD that had a pi/2 phase change at $535 \mathrm{~nm}$ was used. Figure 7.8 a shows the separation distance of the DHD versus wavelength at 635,535 and $450 \mathrm{~nm}$.

The $x$-axis of Figure 7.8 is given in unit steps which are $10 \mu \mathrm{m}$ per step. The distance range is $1-5 \mathrm{~mm}$. Each wavelength curve had its own minimum at which speckle contrast is least. This information allows for monochromatic speckle reduction optimization and can be used to roughly determine the best location for DHD separation with all three wavelengths combined. This distance value can be confirmed by simulating all three diffusers identically across a grayscale and RGB detector. Figure $7.8 \mathrm{~b}$ demonstrates both detection method curves for an RGB speckle image. The speckle contrast value can be further reduced $5 \%$ by increasing the separation distance between the diffusers. Figure 7.8a explains the reason behind the change is because of the dramatic decrease in speckle for the $450 \mathrm{~nm}$ wavelength diffuser curve. It is of interest that the 535 and $635 \mathrm{~nm}$ curves match one another in shape with the main difference being a $6-8 \%$ contrast increase yet the $450 \mathrm{~nm}$ curve starts quite high and drops until it appears to level with the $535 \mathrm{~nm}$ line. Increasing the separation distance of the diffuser spreads out the speckle spots from the first diffuser to the second. The $450 \mathrm{~nm}$ wavelength, at the lowest visible wavelength, has the smallest diffractive output angle. The smaller angle of the output intensity pattern means more condensed speckle spots being projected onto the $2^{\text {nd }}$ diffuser. A shorter separation distance between the diffusers means the $2^{\text {nd }}$ diffuser is essentially underfilled. As separation distance increases, the speckle pattern incident on the $2^{\text {nd }}$ diffuser 102 
gets larger and the contrast decreases. This identifies a very obvious speckle contrast minimum that can be achieved by setting the diffuser spacing. This allows a speckle contrast value of $0.18(+/-0.04)$ to be realized.

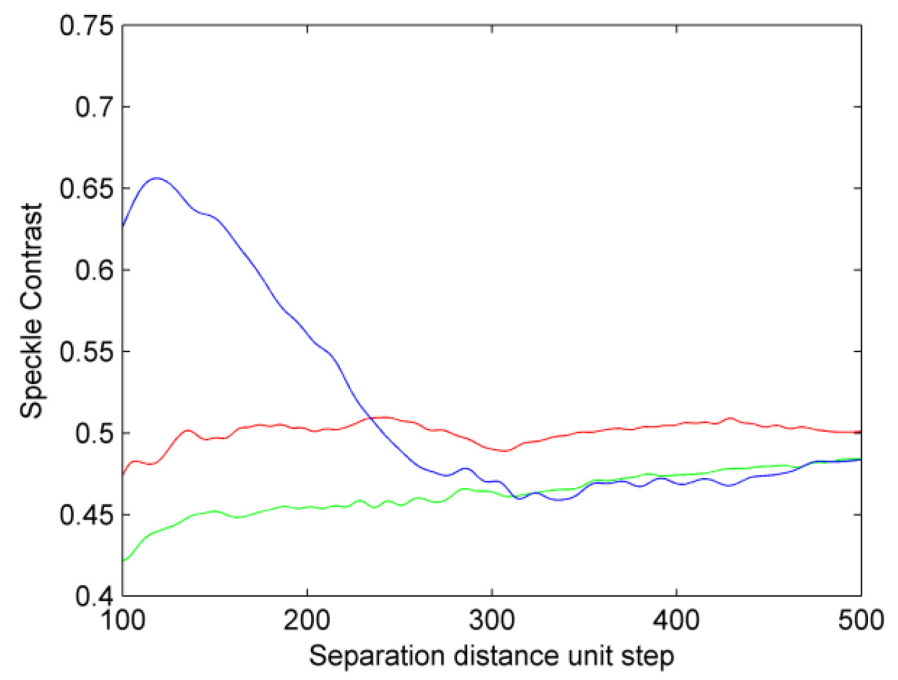

a)

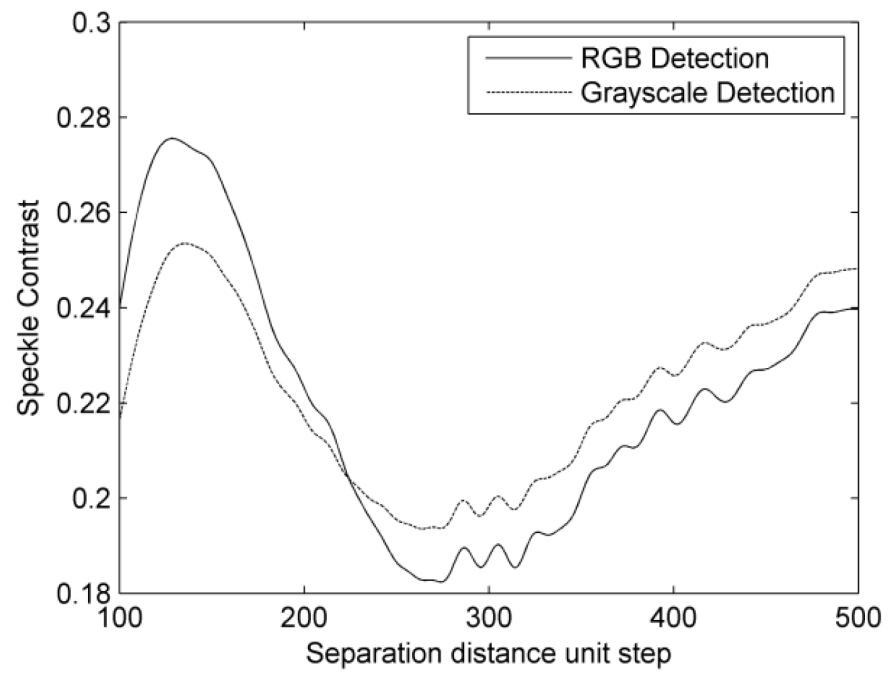

b)

Figure 7.8: Separation distance between the DHD for all three wavelengths:

a) at $635 \mathrm{~nm}, 535 \mathrm{~nm}$ and 450; b) Full color using grayscale and RGB detection 


\subsection{Polychromatic Speckle Reduction Conclusion}

Diffuser based speckle reduction systems are highly flexible to additions, such as the secondary speckle reduction techniques discussed in Chapter 3. A split polarization setup could be added to the system for each laser, allowing for up to $50 \%$ speckle reduction based on previous investigations. However, the complexity associated with such a system would be too elaborate for the current objective of pico-projection. A compromise could be made using a single split-polarization system if the three RGB laser diodes were aligned with the same linear polarization. In addition, the introduction of a low NA fiber optic could further reduce speckle contrast by $20-30 \%$ and allow for reduction in the complexity by using fiber optics for beam steering. The incorporation of secondary speckle reduction methods remains a theoretical argument, but the options are readily available and the previous analysis made in Chapter 3 supports the conclusions made in this chapter. 


\section{Chapter 8: Conclusion}

\subsection{Summary}

This dissertation presents an analysis of the application of diffractive diffusers for reducing speckle in pico-projector electronics. Two types of rotating diffusers were tested and compared with varying wavelength, diffuser speed, and laser beam size. A minimum speckle contrast of 0.18 was achieved utilizing the rotational method. Secondary speckle reduction techniques were examined and tested both alone and in conjunction with the rotating diffusers. An analysis of the practicality of incorporating these speckle reduction systems with diffusers was discussed. Results and conclusions from the experimental diffuser testing were dissected in order to propose and develop new diffractive diffuser designs.

A working mathematical model was developed to model diffractive diffusers using Fourier optics. The diffuser simulation was verified by comparing the results to the experimentally measured values from the rotating diffusers. The modeled diffusers have been shown to be within $10 \%$ of experimental results. Having the ability to perform multi-wavelength analysis of diffractive diffuser performance has also been shown with the model and verified with experimental results. The simulation was then expanded to include projection optics and the ability to model multiple diffusers inline along the optical axis was added. The fully developed model was able to analyze new diffuser designs while eliminating high manufacturing costs and long lead times. 
An optical speckle reduction system was designed that uses multiple stationary (nonrotating) Hadamard matrices in tangent for increased speckle reduction. Hadamard diffusers have been previously analyzed and it has been shown that they can be optimized for speckle reduction. By utilizing multiple specialized Hadamard matrices in tangent, speckle can be further reduced than while alleviating the need for moving parts. A monochromatic speckle contrast measurement of 0.40 has been achieved. Using this type of structured binary matrix will maintain partial coherence of the laser source unlike static pseudo-random grayscale style diffusers. Rotating components provide a mechanical failure mode that should be avoided, if possible.

The Hadamard diffusers have been optimized for polychromatic speckle reduction. Full speckle measurements have been completed for an RGB projector system. Testing has revealed a multi-spectral speckle contrast result of 0.18 . This dissertation has shown that a full color pico-projector speckle reduction system can be accomplished with no rotating or vibrating components. Secondary speckle reduction methods could theoretically be added to the system to allow for a further maximum speckle contrast decrease of 50\%. While lower speckle contrast has been achieved through other methods, the proposed system within this dissertation is easily adaptable and could be effortlessly integrated into any handheld pico-projector device. 


\subsection{Future Work}

Work is needed to integrate the dual-Hadamard diffuser design into a developed projector system. Analysis needs to be completed concerning whether the diffuser design is useable in a MLA based or scanning pico-projector design. Testing the diffuser with a projector would require subsystem development of video display technology and outside the optical engineering discipline.

In addition, more experimentation is needed to determine the ability for the diffuser to reduce speckle under polychromatic illumination as only three wavelengths have been analyzed. Simulation results are needed for wavelengths outside of 635,535 and 450 $\mathrm{nm}$. Full testing of the diffuser using all known visible wavelengths, $400-700 \mathrm{~nm}$ were not completed due to lack of available processing power. Continuation of the simulation to finish this testing is easily accomplished if access to a large computer array for a 3-4 week period is available. This work would show possible wavelengths that allow for lower contrast to optimize any laser projector design. 


\section{References}

1. Hecht, E., Optics. 4th ed. 2002: Pearson Education, Inc.

2. $\quad$ Silfvast, W.T., Laser Fundamentals. 2nd ed. 2004, New York: Cambridge University Press. 642.

3. Dainty, J.C., Laser speckle and related phenomena. 2nd ed. Topics in Applied Physics, ed. J.C. Dainty. 1984: Springer-Verlag. 342.

4. Rigden, J.D. and E.I. Gordon, The granularity of scattered optical maser light. Proceedings of the IRE, 1962. 50(11): p. 2367-2368.

5. Goodman, J.W., Speckle Phenomena in Optics: Theory and Applications. 1st ed. . Vol. . 2007, Englewood: Roberts \& Company. 387.

6. Loewen, E.G. and E. Popov, Diffraction Gratings and Their Applicaitons. 1st ed. Optical Science and Engineering, ed. M. Dekker. Vol. 58. 2000: CRC Press. 630.

7. Palmer, C. and W.R. McKinney, Equivalence of focusing conditions for holographic and varied line-space grating systems. Applied Optics, 1990. 29(1): p. 47-51.

8. Loewen, E., Diffraction Grating Handbook, C. Palmer, Editor. 2005, Newport Corp.: Rochester, NY. p. 265.

9. Goodman, J.W., Statistical Optics. Wiley Classics Library. 2000: John Wiley \& Sons. 550.

10. Livingstone, M., Vision \& Art: The Biology of Seeing. 2002, New York: Harry N. Abrams.

11. Trisnadi, J.I. Speckle contrast reduction in laser projection displays. in Projection Displays VIII. 2002. San Jose: SPIE.

12. Kubota, S. and J.W. Goodman, Very efficient speckle contrast reduction realized by moving diffuser device. Appl. Opt., 2010. 49(23): p. 4385-4391. 
13. Tran, T.K.T., et al., Design, Modeling, and Characterization of a Microelectromechanical Diffuser Device for Laser Speckle Reduction. J. Microelectromech. Syst., 2014. 23(1): p. 117-127.

14. Gao, W., Shuyuan Ma, Xuyuan Chen, Speckle reduction in line scan laser display system by static diffuser with 2D binary code. Chinese Optics Letters, 2012. 10(N/A): p. 4.

15. Bartula, R.J. and S.T. Sanders, Estimation of signal noise induced by multimode optical fibers. Optical Engineering, 2008. 47(3): p. 035002035002-6.

16. Ha, W., et al., Acousto-optic control of speckle contrast in multimode fibers with a cylindrical piezoelectric transducer oscillating in the radial direction. Opt. Express, 2009. 17(20): p. 17536-17546.

17. Zhang, Y.F., et al., Demonstration of a home projector based on $R G B$ semiconductor lasers. Appl. Opt., 2012. 51(16): p. 3584-3589.

18. Smith, J.G. and A. Stockham. Diffractive, aspheric, or spherical beam transformers: a comparison in manufacturability. 2008.

19. Stockham, A. and J.G. Smith. Comparison between a super Gaussian and a "true" top hat. 2008.

20. Poynton, C., Digital Video and HDTV: Algorithms and Interfaces. 1st ed. The Morgan Kaufmann Series in Computer Graphics. 2003: Morgan Kaufmann. 736.

21. Goodman, J.W., Introduction to Fourier Optics. 3rd ed. 2005, Englewood: Roberts \& Company.

22. Papoulis, A. and S.U. Pillai, Probability, Random Variables, and Stochastic Processes. 4th ed. McGraw-Hill series in electrical engineering: Communications and signal processing. 2002, New York: Tata McGraw-Hill. 852 .

23. Manni, J.G. and J.W. Goodman, Versatile method for achieving 1\% speckle contrast in large-venue laser projection displays using a stationary multimode optical fiber. Opt. Express, 2012. 20(10): p. 11288-11315.

24. Roelandt, S., et al., Standardized speckle measurement method matched to human speckle perception in laser projection systems. Optics Express, 2012. 20(8): p. 8770-8783. 
25. Tom, W.J., A. Ponticorvo, and A.K. Dunn, Efficient Processing of Laser Speckle Contrast Images. Ieee Transactions on Medical Imaging, 2008. 27(12): p. 1728-1738.

26. Kirkpatrick, S.J., D.D. Duncan, and E.M. Wells-Gray, Detrimental effects of speckle-pixel size matching in laser speckle contrast imaging. Opt. Lett., 2008. 33(24): p. 2886-2888.

27. Edmund Optics. High Performance Green Laser Diode Modules | Edmund Optics. [cited 2014; Available from:

http://www.edmundoptics.com/lasers/laser-diode-modules/continuous-wavelaser-diode-modules/high-performance-green-laser-diode-modules/2661.

28. Melles Griot. Helium Neon Lasers. [cited 2014; Available from: http://mellesgriot.com/products/Lasers/Helium-Neon-Lasers.

29. Wang, L.L., et al., Speckle reduction in laser projection systems by diffractive optical elements. Appl. Opt., 1998. 37(10): p. 1770-1775.

30. Newport. Beam Shaping with Cylindrical Lenses. Newport Technical Notes [cited 2014; Available from: http://www.newport.com/Beam-Shaping-withCylindrical-Lenses/144888/1033/content.aspx.

31. Clark, T. and K. Wanser, Choosing Ball or Grin Lenses. 2001.

32. Gagliardi, R.M. and S. Karp, Optical Communications. 2nd ed. Wiley Series in Telecommunications and Signal Processing, ed. J.G. Proakis. 1995, New York: John Wiley \& Sons. 347.

33. Thomas, W. and C. Middlebrook. Collimation of asymmetric laser diode at $450 \mathrm{~nm}$ using a multimode fiber for speckle reduction through diffractive diffusers. in Advances in Display Technologies II. 2012. San Fransisco, CA: SPIE.

34. Thomas, W., C. Middlebrook, and J. Smith. Laser speckle contrast reduction measurement using diffractive diffusers. in Emerging Liquid Crystal Technological IV. 2009. San Jose: Proceedings of SPIE.

35. Ouyang, G., et al., Laser speckle reduction based on angular diversity induced by Piezoelectric Benders. J. Eur. Opt. Soc. RP, 2013. 8: p. 4.

36. Voelz, D., Computational Fourier Optics: A MATLAB Tutorial. SPIE Tutorial Texts. Vol. TT89. 2011, Bellingham: SPIE Press. 250.

37. Kurtz, C.N., H.O. Hoadley, and J.J. Depalma, Design and synthesis of random phase diffuser. J. Opt. Soc. Am., 1973. 63(9): p. 1080-1092. 
38. Gremaux, D.A. and N.C. Gallagher, Limits of Scalar Diffraction Theory for Conducting Gratings. Appl. Opt., 1993. 32(11): p. 1948-1953.

39. Harvey, J.E., Fourier Treatment of near-Field Scalar Diffraction Theory. Am. J. Phys., 1979. 47(11): p. 974-980.

40. Verdeyen, J.T., Laser Electronics. 3rd ed. Prentice Hall Series in Solid State Physical Electronics, ed. J. Nick Holonyak. 2000, Saddle River: Prentice Hall. 778.

41. Poon, T.-C. and T. kim, Engineering Optics with MATLAB. 2006, Hackensack: World Scientific.

42. Loui, H., Fourier propagation, in Numerical Methods in Photonics Project. 2004, University of Colorado: Boulder. p. 1-5.

43. Nakayama, Y. and M. Kato, Diffuser with Pseudorandom Phase Sequence. J. Opt. Soc. Am., 1979. 69(10): p. 1367-1372.

44. Agaian, S., et al., Hadamard Transforms. 1st ed. Vol. PM207. 2011, Bellingham, Washington: SPIE Press. 502.

45. Trisnadi, J.I., Hadamard speckle contrast reduction. Opt. Lett., 2004. 29(1): p. 11-13.

46. Gao, W., Vladimir Kartashov, Guangmin Ouyang, Zhaomin Tong, Muahmmed Nadeem Akram, Xuyuan Chen. The matrices used to reduce speckle in laser projector system. in Speckle 2010: Optical Metrology. 2010. Florianapolis Brazil: SPIE.

47. Thomas, W. and C. Middlebrook, Speckle Reduction in Imaging Projection Systems. Opt. Photonics J., 2012. 2(4): p. 6.

48. Thomas, W. and C. Middlebrook, Non-moving Hadamard matrix diffusers for speckle reduction in laser pico-projectors. J. Mod. Opt., 2014: p. 10.

49. Mack, C.A., Field Guide to Optical Lithography. SPIE Press Book. Vol. FG06. 2006: SPIE Publications. 136. 


\section{Appendix A: Hadamard Matrices}

A Hadamard matrix is a mathematical tool used for completing a Hadamard Transform (HT) and are commonly used in signal processing due to their fast processing speeds [44]. A HT is an image processing technique in the same family as the Fourier transform. The benefit of the HT over other methods is that the process only uses subtractive and additive manipulations allowing for faster systems. The HT is accomplished through the use of a Hadamard matrix, consisting of only +1 and -1 . The Hadamard matrix provides a simple, binary structure which can easily be recreated on a glass diffuser in less time than other non-binary designs. The formal definition of a Hadamard matrix is seen in Equation A. 1 where $H$ represents the matrix itself, $n$ is the order of the matrix and $I$ is the Identity Matrix [44].

$$
H_{n} H_{n}^{T}=H_{n}^{T} H_{n}=n I_{n}
$$

The definition in Equation A.1 requires all Hadamard matrices to be square with orders $\mathrm{n}=2,4,8,16, \ldots$ where $H_{1}=[1]$. Three distinct Hadamard matrices were simulated and investigated. The first is the Sylvester matrix which can be constructed through Equation A.2. It is commonly used with Hadamard transforms for signal processing, taking advantage of the faster processing time [44]. An order 16 Sylvester matrix is shown in Figure A.1a where the dark area denotes +1 and the white space denotes -1 . 


$$
H_{2^{n}}=\left(\begin{array}{cc}
H_{2^{n-1}} & H_{2^{n-1}} \\
H_{2^{n-1}} & -H_{2^{n-1}}
\end{array}\right)
$$

$2^{\mathrm{n}}$ is the order size of the matrix and $n$ must a multiple of 2 . The second matrix type is known as a Walsh-Paley matrix can be constructed through recursion as shown in Equation A.2. The order 16 matrix is shown in Figure A.1b.

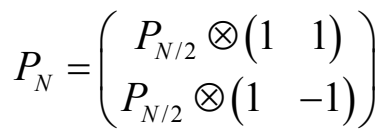

where $P$ is the Walsh-Paley matrix of order $N=2^{\wedge} n$ and $\otimes$ denotes the Kronecker product.

The final matrix type under investigation is the Walsh system and can also be constructed recursively as depicted in Equation Equation A.3. The order 16 Walsh matrix is shown in Figure A.1c.

$$
W_{N}=\left[W_{2} \otimes A_{1},\left(W_{2} R\right) \otimes A_{2}, \ldots, W_{2} \otimes A_{(N / 2)-1},\left(W_{2} R\right) \otimes A_{(N / 2)}\right](\text { Eq. A.3) }
$$

where $W_{2}=\left(\begin{array}{cc}1 & 1 \\ 1 & -1\end{array}\right), R=\left(\begin{array}{ll}0 & 1 \\ 1 & 0\end{array}\right)$ and $A_{i}$ represents the ith column of an order $N=2^{n}$ Walsh matrix.

Rotation or movement of these Hadamard diffusers will create temporal averaging and an overall decrease in the speckle contrast. A sixteen order matrix will be created for each type and duplicated to expand filling the total size of the array. Replicating 
the base matrix across the diffuser will allow for spatial averaging. The Sylvester style diffuser is shown in Figure A.2. Contrast measurements will be taken for both stationary and rotational versions of the matrices and compared. Other statistics will be evaluated to support the contrast results and will be discussed later on.

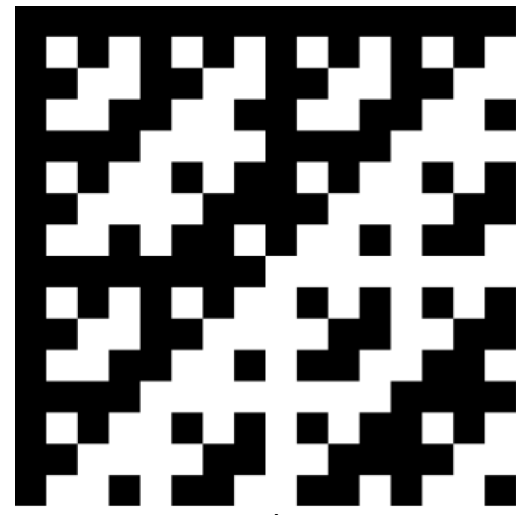

a)

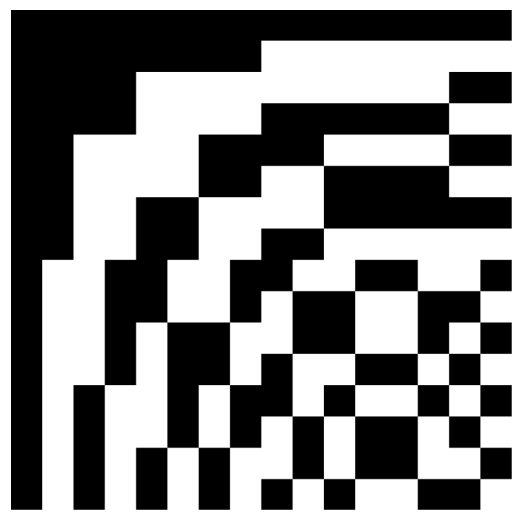

b)

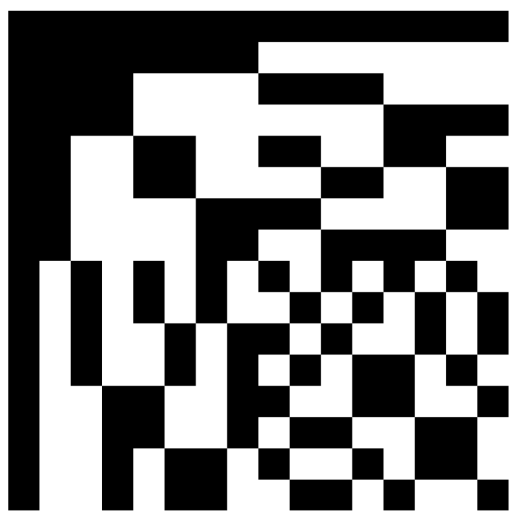

c)

Figure A. 1: Order 16 a) Sylvester matrix, b) Walsh-Paley matrix, c) Walsh Matrix 


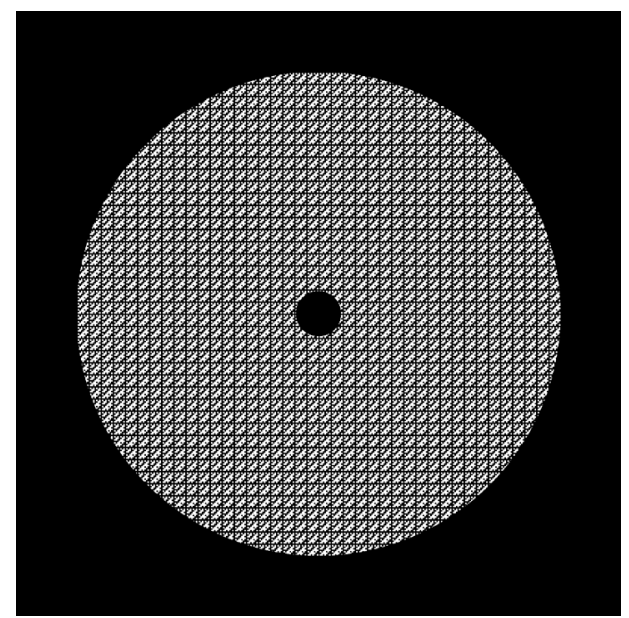

Figure A. 2: Sylvester matrix circular diffuser

A Gaussian beam was projected incident onto both stationary and rotational versions of the diffuser apertures and then propagated out to the near-field image plane following Equation 5.4. Calculations were made for the speckle contrast of stationary and moving diffuser images using Equation 3.2. Full images of the three propagated Hadamard diffusers models from the simulation are shown in Figure A.3. Each matrix type produces a distinct speckle pattern in the near-field. Contrast values for the three patterns are shown in Table A. Rotating versions of the three diffuser models are shown in Figure A.4. Additional speckle contrast values for these patterns are also depicted in Table A.

Table A: Speckle contrast results for various simulations

\begin{tabular}{lll}
\hline Diffuser Type & Stationary & Rotating \\
\hline Sylvester & 0.95 & 0.1758 \\
Walsh-Paley & 0.95 & 0.1767 \\
Walsh & 0.94 & 0.1768 \\
\hline In-line Diffusers & 0.68 & 0.1065 \\
\hline
\end{tabular}



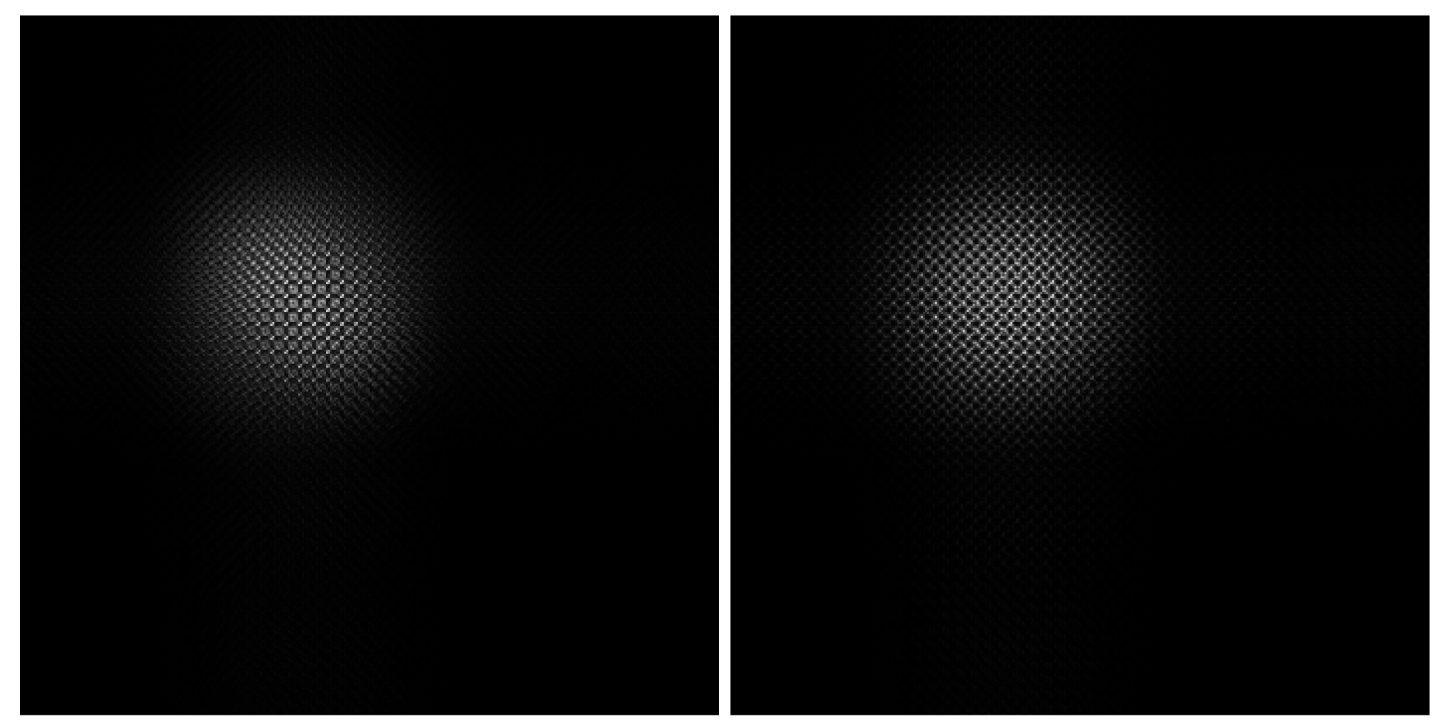

a)

b)

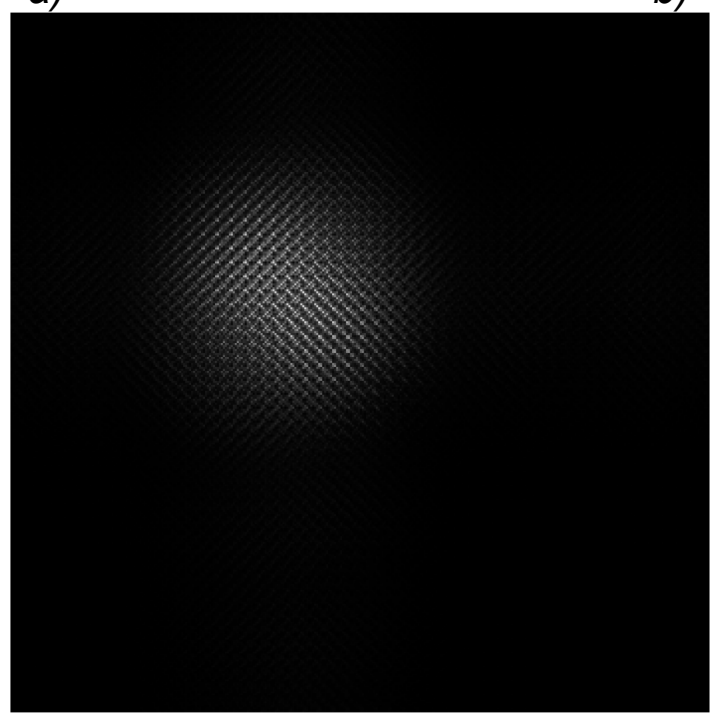

c)

Figure A. 3: a) Sylvester matrix diffuser image; b) Walsh-Paley matrix diffuser image; c) Walsh matrix diffuser image 

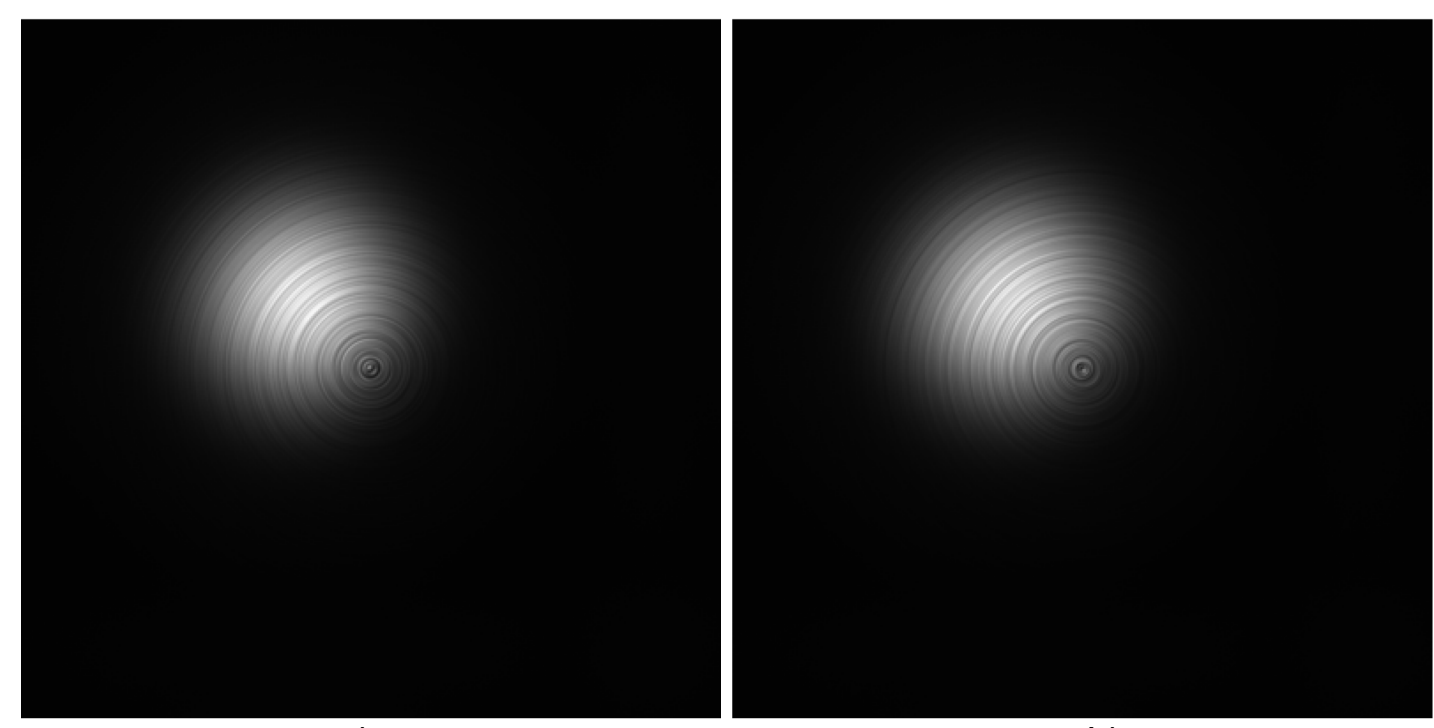

a)

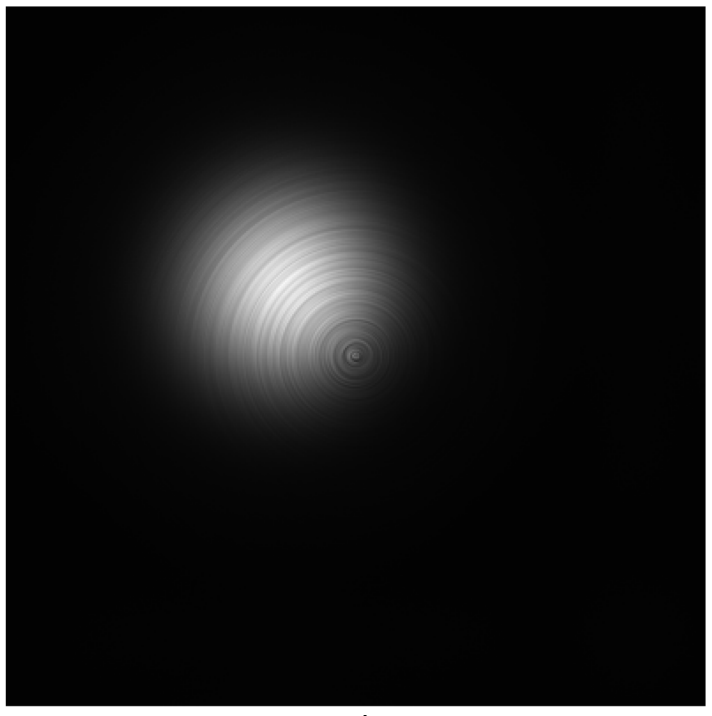

c)

Figure A. 4: Rotating (60 cycles/s) a) Sylvester diffuser, b) Walsh-Paley diffuser, c) Walsh Diffuser 


\title{
Appendix B: MATLAB Code for Monochromatic Dual Hadamard Diffuser Model
}

$\% \%$ Hadamard Matrix Speckle Reduction - Single Wavelength

\begin{abstract}
$\%$ Weston Thomas
\%Primary code used to run diffuser design simulation/model. Other

$\%$ functions are called as needed. All images are saved and exported via $\%$.mat extension (Matlab file storage format). After simulation is finished $\%$ function is called to calculate contrast values from images as needed.
\end{abstract}

\%Date of original code: September 14th, 2012

\%Edited for cluster usage on: January 15th, 2014

\%Formatted for dissertation on: September 10th, 2014

clear, clc

lambda $=535 \mathrm{e}-9$

\%Define initial gaussian beam structure

$\mathrm{g}=$ Gaussian_Beam_2d(.05,8000,8000,.4,.4);

$\%$ Calls pre-defined diffuser structure

tb_diff_one = load('Had_Array_Had_pi.mat');

\%Calls secondary diffuser structure (when needed)

$\%$ tb_diff_two = binary_had $(2,8000,25)$; 
$\mathrm{tb} \_1 \mathrm{c}=1^{*} \mathrm{tb} \_1 . \mathrm{d}$

$\% \%$ Sylvester (Hadamard-Walsh) - Gaussian

\%Gaussian wavefront incident on first diffuser

tb_intial_1=g."tb_diff_one;

$\%$ First propagation of output wavefront

tb_TF_1 = Fres_Prop_TF(tb_ampl_1,10,lambda,1e2);

$\%$ Second diffuser incidence

tb_initial_2 = tb_TF_1.*tb_diff_one;

$\%$ Second propagation of output wavefront

tb_TF_2 = Fres_Prop_TF(tb_ampl_2,10,lambda,1e4);

\%Diffuser output wavefront incident on lens

tb_FF_1 = Fraun_Prop(tb_TF_2,100,lambda,5e3);

$\%$ Final expansion from focal point of lens to image plane tb_TF_3 $=$ Fres_Prop_TF(tb_FF_1,10,lambda,1e5);

\%Amplitude to Intensity manipulation (assume ideal)

tb_intTF_1 = abs(tb_TF_1). ${ }^{\wedge} 2$;

tb_intTF_2 = abs(tb_TF_2). ${ }^{\wedge} 2$;

tb_intFF_1 = abs(tb_FF_1).^2;

tb_intTF_3 = abs(tb_TF_3). ${ }^{\wedge}$;

$\% \%$ Output Data - Normalization and Save

B-2 
\%Intensity normalization for image file type double (needed to export

$\%$ images to .tif format)

$$
\begin{aligned}
& \text { tb_intTF_norm_1 }=\text { tb_intTF_1/max }\left(\max \left(t b \_i n t T F \_1\right)\right) \text {; } \\
& \text { tb_intTF_norm_2 }=\text { tb_intTF_2 } / \max \left(\max \left(t b \_i n t T F \_2\right)\right) \text {; } \\
& \text { tb_intTF_norm_3 }=\text { tb_intTF_3/max }\left(\max \left(t b \_i n t T F \_3\right)\right) \text {; }
\end{aligned}
$$

save('Dual_Diffuser_011714.mat','tb_intTF_1','tb_intFF_1','tb_intTF_2','tb_int TF_3','color_final');

function [output_field] = Fres_Prop_TF(input_field,L_side,lambda,z);

$\%$ Fresnel propagation using transfer function approach

\%Written by Weston Thomas on: June 1st, 2011

\%Edited for dissertation formatting on: September $11^{\text {th }}, 2014$

$\% \%$ Propagation Model

\%Defines grid size

$[\mathrm{M}, \mathrm{N}]=$ size $($ input_field $)$;

$\%$ Defines step size

delta_x $=$ L_side/M;

$\%$ Defines wavenumber

$\mathrm{k}=2^{*} \mathrm{pi} / \mathrm{lambda}$;

$\%$ Defines the output scaled vector (assumes $x$ and $y$ equal linear

$\%$ components i.e. $M=N$, delta_ $x=$ delta_y so $f x=f y$

$f x=-1 /\left(2^{*} d x\right): 1 / L: 1 /\left(2^{*} d x\right)-1 / L$; 
$\%$ Creates output axis grid

$[\mathrm{FX}, \mathrm{FY}]=\operatorname{meshgrid}(\mathrm{fx}, \mathrm{fx})$;

\%Creates transfer function $\mathrm{H}$

$h=\exp \left(1 i^{*} k^{*} Z\right)^{*} \exp \left(-1 i^{*} \mathrm{pi}^{*} \mid a m b d a^{*} Z^{*}\left(F X .^{\wedge} 2+F Y .{ }^{\wedge} 2\right)\right)$;

H_FT = fftshift(h);

input_field_FT $=\mathrm{fft} 2(\mathrm{fftshift}($ input_field));

output_field_FT $=$ H_FT. ${ }^{*}$ input_field_FT;

output_field = ifftshift(ifft2(output_field_FT));

end

function [output_field,L_final] = Fraun_Prop_FF(input_field,L_initial,lambda,z);

$\%$ Fraunhofer propagation code using fft

\%Defines initial grid/matrix size

$[\mathrm{M}, \mathrm{N}]=$ size (input_field);

\%Defines grid spacing (step size) for input field

delta_x1 = L_initial/M;

\%Defines wavenumber

$\mathrm{k}=2^{*} \mathrm{pi} / \mathrm{lambda}$;

\%Calculates final image scaling and step size

$L_{-}$final $=$lambda* $z /$ delta_x1;

delta_x2 = lambda* $z /$ L_intial; 
\%Defines final image axis $\mathrm{M}=\mathrm{N}$, delta_x1=delta_y1 so $\mathrm{fx}=\mathrm{fy}$

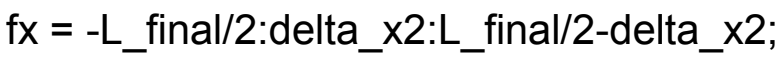

$[\mathrm{FX}, \mathrm{FY}]=\operatorname{meshgrid}(\mathrm{fx}, \mathrm{fx})$;

$\%$ Calculates the output field (with constants)

const $=1 /\left(1 i^{*} \mid a m b d a^{*} z\right)^{*} \exp \left(1 i^{*} k /\left(2^{*} z\right)^{*}\left(F X .^{\wedge} 2+F Y .{ }^{\wedge} 2\right)\right)$;

output_field $=$ const. ${ }^{*}$ ifftshift(fft2(fftshift(input_field))) $)^{*}$ delta_ $\times 11^{\wedge} 2$;

end

function [gauss_field] $=$

Gaussian_Beam_2d(w_0,pixel_x,pixel_y,length_x,length_y);

\%2D Gaussian beam based on amplitude; All lengths are in meters

$\%$ w_0 = beam waist

$\%$ pixel_x \& pixel_y $=$ number of pixels in $\mathrm{x}$ and $\mathrm{y}$ directions

\%length_x \& length_y $=$ physical length of sides in $\mathrm{x}$ and $\mathrm{y}$ directions

$\%$ Sample spacing is a fixed ratio of length/pixel in this function

\%Created By: Kyle Drexler 2010

\%Edited by Weston Thomas on: June 5th, 2011

\%Formatted for dissertation on: Sept 13th, 2014

$\%$ Sample spacing (step size) of beam

delta_x = length_x/pixel_x;

delta_y = length_y/pixel_y;

\%Final image axis grid creation 
$[x y]=$ meshgrid(-pixel_x/2:pixel_x/2-1,-pixel_y/2:pixel_y/2-1);

\%Mapping the 2-d Gaussian image to the created axis gauss_field $=\exp \left(-\left(\left((x) \cdot{ }^{*}\right.\right.\right.$ delta_x $) \cdot{ }^{\wedge} 2+\left((y) \cdot{ }^{*}\right.$ delta_y $\left.\left.) \cdot{ }^{\wedge} 2\right) /\left(w \_0^{\wedge} 2\right)\right)$; end

function $[\mathrm{C}]=$ contrast_image(image_double);

\%Function designed to calculate the contrast of an image by \%taking the average intensity of the image and the standard \%deviation

$\%$ Calculates preliminary sum of the rows of the image matrix Row_Sum = sum(image_double);

$\%$ Calculates the total sum of all values in the image matrix Total_Sum =sum(Row_Sum);

$\%$ Maps the size of the image matrix $[\mathrm{kl}]=$ size(image_double);

$\%$ Total number of pixels in the image image_sum $=k^{*}$;

$\%$ The total value of all pixels divided by the total number of \%pixels Avg = Total_Sum/image_sum;

\%Preallocating a new image to use for the calculation of the \%standard deviation

B-6 
New_Double = 0;

\%Double for loop to calculate the standard deviation of the image for $m=1: k$;

for $n=1: l$;

New_Double $=$ New_Double+(image_double $(m, n)-A v g) .{ }^{\wedge}$;

end

end

$\%$ Calculates the contrast of the image by dividing the standard

$\%$ deviation by the average intensity

C = sqrt(New_Double/(image_sum))/Avg;

end 


\section{Appendix C: MATLAB Code for Polychromatic Dual Hadamard Diffuser Model}

The following code creates polychromatic speckle images for contrast measurement.

The main function is displayed here while sub-routine functions that are used can be found in Appendix B.

$\% \%$ Hadamard Matrix Speckle Reduction

function[r,gr,b,rgb,rgbg,rgbi] = hadamard_cluster_rgb()

$\%$ Weston Thomas

\%Primary code for polychromatic speckle contrast measurements. Three wavelengths \%were the focus for this function, although more can be included. All output data \%matrices are saved in .mat format for integrity. Contrast measurements are calculated \%through a separation sub-routine function.

\%Date of original code: November 4th, 2013

\%Edited for cluster usage on: January 15th, 2014

clear, clc, close all

\% Defines simulating wavelengths (RGB)

lambda_r = 635e-9;

lambda_g = 535e-9;

lambda_b = 450e-9; 
$\%$ Define initial gaussian beam structure

$\mathrm{g}=$ Gaussian_Beam_2d(.05,8000,8000,.4,.4);

$\%$ Calls pre-defined diffuser structure

$\%$ Unique diffuser models are needed for 635 and $450 \mathrm{~nm}$ wavelengths to match phase

\%variation due to physical silica etch parameters

tb_diff_r = load('Had_Array_Had_635_pi.mat');

tb_diff_g = load('Had_Array_Had_pi.mat');

tb_diff_b = load('Had_Array_Had_450_pi.mat');

\%Calls secondary diffuser structure (when needed)

$\%$ tb_diff_two = binary_had $(2,8000,25)$;

\%Identifies parameters for the propagation distance between the diffusers $\%$ (separation) and the focus distance that creates the lens propagation

$\%$ (focus)

focus $=1 \mathrm{e} 5$

separation $=1 \mathrm{e} 2$;

$\% \%$ Sylvester (Hadamard diffuser propagation) - Gaussian amplitude (635 $\% \% \mathrm{~nm})$

\%Gaussian wavefront incident on first diffuser

tb_intial_1 $r=g .{ }^{*}$ tb_diff_r;

$\%$ First propagation of output wavefront tb_TF_1r $=$ Fres_Prop_TF(tb_initial_1 $r, 10$,lambda_r, 1e2); 
\%Second diffuser incidence

tb_initial_2 $r=t b \_T F \_1 r .{ }^{*} t b \_d i f f \_r$;

$\%$ Second propagation of output wavefront

tb_TF_2 $r=$ Fres_Prop_TF(tb_initial_2r,10,lambda_r,separation);

\%Diffuser output wavefront incident on lens

tb_FF_1r = Fraun_Prop(tb_TF_2r,100,lambda_r,5e3);

\%Final expansion from focal point of lens to image plane

tb_TF_3r $=$ Fres_Prop_TF(tb_FF_1r,10,lambda_r,focus);

$\%$ Amplitude to Intensity manipulation (assume ideal)

tb_intTF_3r = abs(tb_TF_3r). ${ }^{\wedge} 2$;

$\% \%$ Sylvester (Hadamard diffuser propagation) - Gaussian amplitude (535

$\% \% \mathrm{~nm})$

\%Gaussian wavefront incident on first diffuser

tb_intial_1g=g.ttb_diff_g;

$\%$ First propagation of output wavefront

tb_TF_1g = Fres_Prop_TF(tb_initial_1g,10,lambda_g,1e2);

\%Second diffuser incidence

tb_initial_2g = tb_TF_1g.*tb_diff_g;

$\%$ Second propagation of output wavefront

tb_TF_2g = Fres_Prop_TF(tb_initial_2g,10,lambda_g,separation); 
\%Diffuser output wavefront incident on lens

tb_FF_1g = Fraun_Prop(tb_TF_2g,100,lambda_g,5e3);

\%Final expansion from focal point of lens to image plane

tb_TF_3g = Fres_Prop_TF(tb_FF_1g,10,lambda_g,focus);

$\%$ Amplitude to Intensity manipulation (assume ideal)

tb_intTF_3g = abs(tb_TF_3g).^2;

$\% \%$ Sylvester (Hadamard diffuser propagation) - Gaussian amplitude (450

$\% \% \mathrm{~nm})$

$\%$ Gaussian wavefront incident on first diffuser

tb_intial_1b=g.*tb_diff_b;

$\%$ First propagation of output wavefront

tb_TF_1b $=$ Fres_Prop_TF(tb_initial_1b,10,lambda_b,1e2);

\%Second diffuser incidence

tb_initial_2b $=$ tb_TF_1b.*tb_diff_b;

$\%$ Second propagation of output wavefront

tb_TF_2b = Fres_Prop_TF(tb_initial_2b,10,lambda_b,separation);

$\%$ Diffuser output wavefront incident on lens

tb_FF_1b = Fraun_Prop(tb_TF_2b,100,lambda_b,5e3);

$\%$ Final expansion from focal point of lens to image plane tb_TF_3b $=$ Fres_Prop_TF(tb_FF_1b,10,lambda_b,focus); 
\%Amplitude to Intensity manipulation (assume ideal)

tb_intTF_3b = abs(tb_TF_3b).^2;

$\% \%$ Output Data - Colormap and Normalization (RGB)

$\%$ Normalization to unity

$r=t b \_i n t T F \_3 r / \max \left(\max \left(t b \_i n t T F \_3 r\right)\right)$;

$\mathrm{gr}=\mathrm{tb} \_$intTF_3g/max $\left(\max \left(\mathrm{tb} \_\right.\right.$intTF_3g $\left.)\right)$;

$b=t b \_i n t T F \_3 b / \max \left(\max \left(t b \_i n t T F \_3 b\right)\right)$;

$\%$ Predefined matrix for color image

$\operatorname{rgb}=\operatorname{zeros}(8000,8000,3)$;

\%Defines each dimension of the color layer

$\operatorname{rgb}(:,:, 1)=r$;

$\operatorname{rgb}(:,,, 2)=g r ;$

$\operatorname{rgb}(:,:, 3)=b$

\%Manipulation of the full color image to grayscale image for contrast measurement

rgbi = rgb2gray $(r g b)$;

\%Predefined matrix for color image averaging

$\operatorname{rgbg}=\operatorname{zeros}(8000,8000)$;

\%Image mimicking grayscale detection method. Adds together on amplitude basis and \%non-similar wavelength based images do not interfere.

rgbg $=$ abs(abs(tb_TF_3r)+abs(tb_TF_3g)+abs(tb_TF_3b)).^2; 
\%Function called to output all image file data and archive into '.mat' file matrix save('Had-Pal_635_031114.mat','tb_intTF_1r','tb_intTF_2r','tb_intTF_3r'); save('Had-Pal_535_031114.mat','tb_intTF_1g','tb_intTF_2g','tb_intTF_3g'); save('Had-Pal_450_031114.mat','tb_intTF_1b','tb_intTF_2b','tb_intTF_3b'); save('Had-Pal_Color_031114.mat','color_final');

end 
The next function is used to model the speckle contrast of the dual diffusers for a large range of distances separating the two diffusers. Once again all non-MATLAB built-in functions are found in Appendix B of this dissertation.

$\% \%$ Hadamard Matrix Speckle Reduction - Distance Between Diffusers

$\%$ Weston Thomas

\%Date of original code: September 14th, 2012

\%Edited for cluster usage on: January 15th, 2014

$\%$ Edited to incorporate RGB and grayscale polychromatic detection on: July \%10th, 2014

clear, clc, close all

$\%$ Defines simulating wavelengths (RGB)

lambda_ $r=635 \mathrm{e}-9$;

lambda_g = 535e-9;

lambda_b = 450e-9;

\%Define initial gaussian beam structure

$g=$ Gaussian_Beam_2d(.05,8000,8000,.4,.4);

$\%$ Calls pre-defined diffuser structure

$\%$ Unique diffuser models are needed for 635 and $450 \mathrm{~nm}$ wavelengths to $\%$ match phase variation due to physical silica etch parameters

tb_diff_r = load('Had_Array_Had_635_pi.mat');

tb_diff_g = load('Had_Array_Had_pi.mat');

tb_diff_b = load('Had_Array_Had_450_pi.mat'); 
\%Calls secondary diffuser structure (when needed)

$\%$ tb_diff_two = binary_had $(2,8000,25)$;

$\%$ Define the maximum distance vector that separates the two diffusers dis $=500$;

\%\% Sylvester (Hadamard diffuser propagation) - Gaussian amplitude (RGB)

\%Multiplication of gaussian amplitude data for first diffuser image

tb_ampl_r $=$ g.*tb_diff_r;

tb_ampl_g = g.*tb_diff_g;

tb_ampl_b = g.*tb_diff_b;

$\%$ Predefines vectors for varying contrast values over the distance of separation between \%diffusers

contrast_curve_gr_full = zeros $(1$, dis $)$;

contrast_curve_rgb_full = zeros $(1$, dis $)$;

contrast_curve_gr_part = zeros $(1$, dis $)$;

contrast_curve_rgb_part = zeros $(1$, dis $)$;

contrast_curve_r $=$ zeros $(1$, dis $)$;

contrast_curve_g = zeros(1,dis);

contrast_curve_b = zeros(1,dis);

\%Identifies parameters for the propagation distance after the diffusers

$\%$ (focus_1) and the focus distance that creates the lens propagation

\%(focus_2)

focus_1 = 1e5; 
focus_2 $=0.9 \mathrm{e} 5$;

$\%$ This function was designed to be used in a super computer cluster where $\%$ the following iterations of the 'for' loop can be run independently from one \%another. Memory is a concern so the large matrices will be deleted after \%each iteration section to hasten the function. Each iteration has the created $\%$ speckle images measured for contrast and the contrast value recorded $\%$ versus the specific distance between diffusers.

for $m=100$ :dis;

\%Gaussian wavefront incident on first diffuser (635 nm)

tb_intial_1 = g.*tb_diff_r;

$\%$ First propagation of output wavefront

tb_TF_1 = Fres_Prop_TF(tb_initial_1,10,lambda_r,m);

$\%$ Second diffuser incidence

tb_initial_2 = tb_TF_1. ${ }^{*}$ tb_diff_r;

$\%$ Second propagation of output wavefront

tb_TF_2 = Fres_Prop_TF(tb_initial_2,10,lambda_r,focus_1);

\%Diffuser output wavefront incident on lens

tb_FF_1 = Fraun_Prop(tb_TF_2,100,lambda_r,5e3);

$\%$ Final expansion from focal point of lens to image plane tb_TF_3 = Fres_Prop_TF(tb_FF_1,10,lambda_r,focus_2); 
\%Amplitude to Intensity manipulation (assume ideal) and saving the $\%$ amplitude data

amp_r = tb_TF_3;

int_r $=$ abs(tb_TF_3). ${ }^{\wedge}$;

$\%$ Clears the unnecessary intermediate propagation matrices to save \%memory

clear tb_TF_1 tb_ampl_2 tb_TF_2 tb_FF_1 tb_TF_3;

$\%$ Gaussian wavefront incident on first diffuser (535 nm)

tb_intial_1=g.*tb_diff_g;

$\%$ First propagation of output wavefront

tb_TF_1 = Fres_Prop_TF(tb_initial_1,10,lambda_g,m);

\%Second diffuser incidence

tb_initial_2 = tb_TF_1.*tb_diff_g;

$\%$ Second propagation of output wavefront

tb_TF_2 = Fres_Prop_TF(tb_initial_2,10,lambda_g,focus_1);

$\%$ Diffuser output wavefront incident on lens

tb_FF_1 = Fraun_Prop(tb_TF_2,100,lambda_g,5e3);

$\%$ Final expansion from focal point of lens to image plane

tb_TF_3 $=$ Fres_Prop_TF(tb_FF_1,10,lambda_g,focus_2); 
\%Amplitude to Intensity manipulation (assume ideal) and saving the $\%$ amplitude data

amp_g $=$ tb_TF_3;

int_g = abs(tb_TF_3). ${ }^{\wedge}$;

$\%$ Clears the unnecessary intermediate propagation matrices to save \%memory

clear tb_TF_1 tb_ampl_2 tb_TF_2 tb_FF_1 tb_TF_3;

$\%$ Gaussian wavefront incident on first diffuser (450 nm)

tb_intial_1=g.*tb_diff_b;

$\%$ First propagation of output wavefront

tb_TF_1 = Fres_Prop_TF(tb_initial_1,10,lambda_b,m);

\%Second diffuser incidence

tb_initial_2 =tb_TF_1.*tb_diff_b;

$\%$ Second propagation of output wavefront

tb_TF_2 = Fres_Prop_TF(tb_initial_2,10,lambda_b,focus_1);

$\%$ Diffuser output wavefront incident on lens

tb_FF_1 = Fraun_Prop(tb_TF_2,100,lambda_b,5e3);

\%Final expansion from focal point of lens to image plane

tb_TF_3 = Fres_Prop_TF(tb_FF_1,10,lambda_r,focus_2); 
\%Amplitude to Intensity manipulation (assume ideal) and saving the $\%$ amplitude data

amp_b = tb_TF_3;

int_b = abs(tb_TF_3). ${ }^{\wedge}$;

$\%$ Clears the unnecessary intermediate propagation matrices to save \%memory

clear tb_TF_1 tb_ampl_2 tb_TF_2 tb_FF_1 tb_TF_3;

$\%$ Creates the grayscale and $\mathrm{rgb}$ detected images

$g r=a b s\left(a b s\left(a m p \_b\right)+a b s\left(a m p \_r\right)+a b s\left(a m p \_g\right)\right) .^{\wedge} 2$;

rgb = int_b+int_r+int_g;

$\%$ Calculates the speckle contrast for ' $m$ ' distance of separation for full color $\%$ and grayscale (speckle is calculated for partial and full image matrices) contrast_curve_gr_full $(\mathrm{m})=$ contrast_image $(\mathrm{gr})$; contrast_curve_rgb_full $(\mathrm{m})=$ contrast_image $(\mathrm{rgb})$;

contrast_curve_gr_part $(\mathrm{m})=$ contrast_image $(\operatorname{gr}(3000: 5000,3000: 5000))$; contrast_curve_rgb_part $(\mathrm{m})=$ contrast_image $(\operatorname{rgb}(3000: 5000,3000: 5000))$;

$\%$ Calculates the speckle contrast for ' $m$ ' distance of separation for \%individual RGB images (speckle is calculated for partial and full image $\%$ matrices)

contrast_curve_r $(\mathrm{m})=$ contrast_image $($ int_r $)$;

contrast_curve_g $(\mathrm{m})=$ contrast_image $($ int_g);

contrast_curve_b $(m)=$ contrast_image $($ int_b); 
contrast_curve_r_part $(\mathrm{m})=$ contrast_image $($ int_r(3000:5000,3000:5000));

contrast_curve_g_part $(m)=$ contrast_image(int_g(3000:5000,3000:5000));

contrast_curve_b_part $(\mathrm{m})=$ contrast_image $($ int_b(3000:5000,3000:5000));

\%Resets remaining matrix data to reduce memory consumption

clear amp_rint_ramp_g int_g amp_b int_b gr rgb;

end

\%Archives the contrast data to be analyzed later.

save('CDC_RGB-GR_7-29-14.mat',

'contrast_curve_gr_full','contrast_curve_rgb_full','contrast_curve_gr_part',

'contrast_curve_rgb_part');

save('CDC_R-G-B_7-29-14.mat',

'contrast_curve_r','contrast_curve_g','contrast_curve_b','contrast_curve_r_par

t', 'contrast_curve_g_part','contrast_curve_b_part'); 